\title{
On the multiple-scale analysis for some linear partial $q$-difference and differential equations with holomorphic coefficients
}

\author{
Thomas Dreyfus ${ }^{1}$, Alberto Lastra ${ }^{2 *}$ id and Stéphane Malek $^{3}$
}

"Correspondence:

alberto.lastra@uah.es

2Departamento de Física y

Matemáticas, University of Alcalá,

Alcalá de Henares, Spain

Full list of author information is

available at the end of the article

\section{Springer}

\begin{abstract}
We consider analytic and formal solutions of certain family of $a$-difference-differential equations under the action of a complex perturbation parameter. The previous study (Lastra and Malek in Adv. Differ. Equ. 2015:344, 2015) provides information in the case where the main equation under study is factorizable as a product of two equations in the so-called normal form. Each of them gives rise to a single level of $q$-Gevrey asymptotic expansion. In the present work, the main problem under study does not suffer any factorization, and a different approach is followed. More precisely, we lean on the technique developed in (Dreyfus in Int. Math. Res. Not. 15:6562-6587, 2015, where the first author makes distinction among the different $q$-Gevrey asymptotic levels by successive applications of two q-Borel-Laplace transforms of different orders, both to the same initial problem, which can be described by means of a Newton polygon.
\end{abstract}

MSC: $35 \mathrm{C} 10 ; 35 \mathrm{C} 20$

Keywords: Asymptotic expansion; Borel-Laplace transform; Fourier transform; Formal power series; Singular perturbation; q-difference-differential equation

\section{Introduction}

This work is devoted to the study of a family of linear $q$-difference-differential problems in the complex domain. It can be arranged into a series of works dedicated to the asymptotic study of holomorphic solutions to different kinds of $q$-difference-differential problems involving irregular singularities such as [5-7, 10], and [12]. The study of $q$-difference and $q$-difference-differential equations in the complex domain is a promising and fruitful domain of research. In the literature, we may find other interesting approaches to these problems. We refer to [20] as a reference, and contributions in the framework of nonlinear $q$-analogs of Briot-Bouquet-type partial differential equations in [21]. We provide [18, 22] as novel studies in this direction.

The study of $q$-difference equations has also been under study in different applications in the last years. Some advances in this respect are [14-16] and the references therein.

(c) The Author(s) 2019. This article is distributed under the terms of the Creative Commons Attribution 4.0 International License (http://creativecommons.org/licenses/by/4.0/), which permits unrestricted use, distribution, and reproduction in any medium, provided you give appropriate credit to the original author(s) and the source, provide a link to the Creative Commons license, and indicate if changes were made. 
The main aim of this work is to study a family of $q$-difference-differential equations of the form

$$
\begin{aligned}
& Q\left(\partial_{z}\right) \sigma_{q, t} u(t, z, \epsilon) \\
& =(\epsilon t)^{d_{D_{1}}} \sigma_{q, t}^{\frac{d_{D_{1}}}{k_{1}}+1} R_{D_{1}}\left(\partial_{z}\right) u(t, z, \epsilon)+(\epsilon t)^{d_{D_{2}}} \sigma_{q, t}^{\frac{d_{D_{2}}}{k_{2}}+1} R_{D_{2}}\left(\partial_{z}\right) u(t, z, \epsilon) \\
& \quad+\sum_{\ell=1}^{D-1} \epsilon^{\Delta_{\ell}} t^{d_{\ell}} \sigma_{q, t}^{\delta_{\ell}}\left(c_{\ell}(t, z, \epsilon) R_{\ell}\left(\partial_{z}\right) u(t, z, \epsilon)\right)+\sigma_{q, t} f(t, z, \epsilon),
\end{aligned}
$$

where $D, D_{1}, D_{2}$ are integer numbers greater than $3, Q, R_{D_{1}}, R_{D_{2}}$, and $R_{\ell}$ for $\ell=1, \ldots, D-1$ are polynomials with complex coefficients, $\Delta_{\ell} \geq 0, \delta_{\ell}, d_{\ell} \geq 1$ are nonnegative integers for $1 \leq \ell \leq D-1, d_{D_{1}}$ and $d_{D_{2}}$ are positive integers, and $q>1$ stands for a real number.

We consider the dilation operator $\sigma_{q, t}$ acting on the variable $t$, that is, $\sigma_{q, t}(t \mapsto f(t)):=$ $f(q t)$, and the generalization of its composition given by

$$
\sigma_{q, t}^{\gamma}(t \mapsto f(t)):=f\left(q^{\gamma} t\right)
$$

for $\gamma \in \mathbb{R}$. We also fix positive integer numbers $k_{1}$ and $k_{2}$ such that

$$
1 \leq k_{1}<k_{2}
$$

As in the previous work [13] of the third author, the coefficients $c_{\ell}(t, z, \epsilon)$ and the forcing term $f(t, z, \epsilon)$ represents a bounded holomorphic function in the vicinity of the origin in $\mathbb{C}^{2}$ with respect to $(t, \epsilon)$ and on a horizontal strip $H_{\beta}=\{z \in \mathbb{C} /|\operatorname{Im}(z)|<\beta\}$ of width $2 \beta>0$ relatively to the space variable $z$. However, a new additional constraint is required on the growth of the Taylor expansion of each $c_{\ell}$ according to the mixed variable $t \epsilon$; see (4.6). It implies that the functions $c_{\ell}(t, z, \epsilon)$ can be extended to entire functions in the monomial $\epsilon t$ in the whole plane $\mathbb{C}$ with so-called $q$-exponential growth of some order related to $k_{1}$ and $k_{2}$ (this terminology will be explained later in the paper).

Two singularly perturbed terms on the right-hand side of equation (1.1) are distinguished. This makes a crucial difference with respect to the previous work [7], in which only one term appears, whilst the multilevel $q$-Gevrey asymptotic behavior comes from the forcing term. More precisely, in that previous work, we focused on families of $q$ difference-differential equations that can be factorized as products of two operators in the so-called normal forms, each enjoying one single level of $q$-Gevrey asymptotics. In the present work, the appearance of these two terms would cause a multilevel $q$-Gevrey phenomenon in the study of the asymptotic solution of (1.1) regarding the perturbation parameter. Our approach is following a two-step procedure of summation of the formal solution, which makes the two $q$-Gevrey asymptotic orders emerge.

Another important difference compared to our previous contribution [7] is that we are now able to handle holomorphic coefficients in time $t$ whilst only polynomial coefficients were considered in [7]. This fact relies on new technical bounds for a $q$-analog of the convolution of order $k$ presented in Proposition 2.6.

The point of view we use here is similar to that performed in the work of the first author (see [3]) and is related to direct constraints on the shape of the main equation via a possible 
description by a Newton polygon. It is important to stress that this approach is specific to the $q$-difference case. Namely, such a direct procedure for producing two different Gevrey levels in the differential case for the problem stated in the work [6] is impossible due to very strong restrictions related to a formula used in the proof and appearing in [19] (see formula (8.7) on p. 3630. In that case, only a proposal via factoring the main equation did actually work, as performed in our joint work [8].

Let us briefly review the steps followed to achieve our main results in the present work. Let $0 \leq p \leq \varsigma-1$. First, we apply the $q$-Borel transformation of order $k_{1}$ to equation (1.1) to obtain our first auxiliary problem in a Borel plane, problem (4.11). A fixed point result in a complex Banach space of functions under an appropriate growth at infinity leads us to an analytic solution $w_{k_{1}}^{\mathfrak{d}_{p}}(\tau, m, \epsilon)$ of $(4.11)$. More precisely, $w_{k_{1}}^{\mathfrak{d}_{p}}(\tau, m, \epsilon)$ defines a continuous function defined in $\left(U_{\mathfrak{d}_{p}} \cup D(0, \rho)\right) \times \mathbb{R} \times D\left(0, \epsilon_{0}\right)$, where $U_{\mathfrak{d}_{p}}$ is an infinite sector of bisecting direction $\mathfrak{d}_{p}$, and holomorphic with respect to the variables $\tau$ and $\epsilon$ in $\left(U_{\mathfrak{o}_{p}} \cup D(0, \rho)\right)$ and $D\left(0, \epsilon_{0}\right)$, respectively. In addition, this function admits $q$-exponential growth of order $\kappa$ at infinity with respect to $\tau$ in $U_{\mathfrak{o}_{p}}$.This result is described in detail in Proposition 4.2.

A second auxiliary problem in the Borel plane is constructed by applying the formal $q$ Borel transformation of order $k_{2}$ to the main problem (1.1). The second auxiliary equation is stated in (4.26). A second fixed point result in another appropriate Banach space of functions allows us to guarantee the existence of an actual solution $w_{k_{2}}^{\mathfrak{o}_{p}}(\tau, m, \epsilon)$ of the second auxiliary problem defined in $S_{\mathfrak{d}_{p}} \times \mathbb{R} \times D\left(0, \epsilon_{0}\right)$ and holomorphic with respect to $\tau$ and $\epsilon$ in $S_{\mathfrak{o}_{p}}$ and $D\left(0, \epsilon_{0}\right)$, respectively. Here, $S_{\mathfrak{d}_{p}}$ stands for an infinite sector with vertex at the origin and bisecting direction $\mathfrak{d}_{p}$. Moreover, this function has $q$-exponential growth of order $k_{2}$ at infinity with respect to $\tau$ on $S_{\mathfrak{d}_{p}}$. This statement is proved in Proposition 4.3.

As a matter of fact, the key point in our reasoning is the link between the $q$-Laplace transforms of $w_{k_{1}}^{\mathrm{d}_{p}}$ and $w_{k_{2}}^{\mathrm{d}_{p}}$ of order $\kappa$ with respect to variable $\tau$. In Proposition 4.4, we guarantee that both functions coincide in the intersection of their domain of definition. This would entail that the function $\mathcal{L}_{q ; 1 / \kappa}^{d}\left(w_{k_{1}}^{d}(\tau, m, \epsilon)\right)$ can be continued along the direction $\mathfrak{d}_{p}$, with $q$-exponential growth of order $k_{2}$; see Propoposition 4.4.

The construction of the analytic solution $u^{\mathrm{d}_{p}}(t, z, \epsilon)$ of $(1.1)$ is obtained after the application of the $q$-Laplace transform of order $k_{2}$ and the inverse Fourier transform, providing a holomorphic function defined in $\mathcal{T} \times H_{\beta^{\prime}} \times \mathcal{E}_{p}$, where $\mathcal{T}$ is some well-chosen bounded sector centered at 0 , and $\left\{\mathcal{E}_{p}\right\}_{0 \leq p \leq s_{-}-1}$ represents a good covering in $\mathbb{C}^{*}$ (see Definition 5.1). This result is described in Theorem 5.3. Figure 1 illustrates the procedure to follow. For the attainment of the asymptotic properties of the analytic solution, we use a Ramis-Sibuyatype theorem in two levels (see Theorem 6.3) and the properties held by the difference of two analytic solutions in the intersection of their domains whenever it is not empty. The conclusion yields two different $q$-Gevrey levels of asymptotic behavior of the analytic

$$
\begin{aligned}
& \text { Figure } 1 \text { Scheme of the different Borel levels } \\
& \text { attained in the construction of the solution }
\end{aligned}
$$

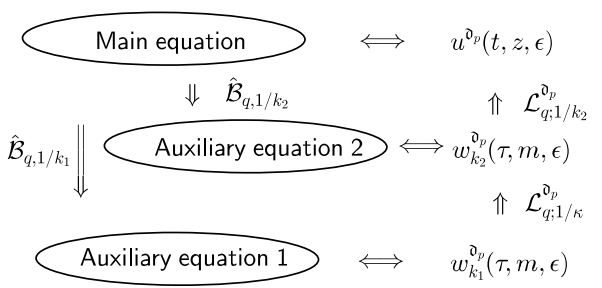


solution with respect to the formal solution depending on the geometry of the problem. The final main result states the splitting of both formal and analytic solutions to the problem under study as a sum of three terms. More precisely, if $\mathbb{F}$ denotes the Banach space of holomorphic and bounded functions defined in $\mathcal{T} \times H_{\beta^{\prime}}$, and $\hat{u}(t, z, \epsilon)$ stands for the formal power series solution of (1.1), then

$$
\hat{u}(t, z, \epsilon)=a(t, z, \epsilon)+\hat{u}_{1}(t, z, \epsilon)+\hat{u}_{2}(t, z, \epsilon)
$$

where $a(t, z, \epsilon) \in \mathbb{F}\{\epsilon\}$ and $\hat{u}_{1}(t, z, \epsilon), \hat{u}_{2}(t, z, \epsilon) \in \mathbb{F} \llbracket \epsilon \rrbracket$ are such that for every $0 \leq p \leq \varsigma-1$, the function $u^{\mathrm{d}_{p}}$ can be written in the form

$$
u^{\mathfrak{d}_{p}}(t, z, \epsilon)=a(t, z, \epsilon)+u_{1}^{\mathfrak{d}_{p}}(t, z, \epsilon)+u_{2}^{\mathfrak{d}_{p}}(t, z, \epsilon)
$$

where $\epsilon \mapsto u_{1}^{\mathfrak{o}_{p}}(t, z, \epsilon)$ is an $\mathbb{F}$-valued function admitting $\hat{u}_{1}(t, z, \epsilon)$ as its $q$-Gevrey asymptotic expansion of order $1 / k_{1}$ on $\mathcal{E}_{p}$, and also $\epsilon \mapsto u_{2}^{\mathrm{o}_{p}}(t, z, \epsilon)$ is an $\mathbb{F}$-valued function admitting $\hat{u}_{2}(t, z, \epsilon)$ as its $q$-Gevrey asymptotic expansion of order $1 / k_{2}$ on $\mathcal{E}_{p}$. This corresponds to Theorem 6.4.

The paper is organized as follows. In Sect. 2.1, we define a weighted Banach space of continuous functions on the domain $(D(0, \rho) \cup U) \times \mathbb{R}$ with $q$-exponential growth on the unbounded sector $U$ with respect to the first variable and exponential decay on $\mathbb{R}$ with respect to the second one. We study the continuity properties of several operators acting on this Banach space. Section 2.2. is concerned with the study of a second family of Banach spaces of functions with $q$-exponential growth on an infinite sector with respect to one variable and exponential decay on $\mathbb{R}$ with respect to the other variable. In Sect. 3, we recall the definitions and main properties of formal and analytic operators involved in the solution of the main equation. Namely, the formal $q$-Borel transformation and analytic $q$-Laplace transform of certain $q$-Gevrey orders, and the inverse Fourier transform. In Sects. 4.1 and 4.2, we study the analytic solutions of two auxiliary problems in two different Borel planes and relate them via $q$-Laplace transformation (see Theorem 5.3). In Sect. 5, we describe in detail the main problem under study and construct its analytic solution and the rate of growth of the difference of two neighboring solutions in their common domain of definition. Finally, in Sect. 6, we deal with the existence of a formal solution of the problem and study the asymptotic behavior relating the analytic and formal solutions through a multilevel $q$-Gevrey asymptotic expansion (Theorem 6.4). This result is attained with the application of a two-level $q$-version of the Ramis-Sibuya theorem (Theorem 6.3).

\section{Auxiliary Banach spaces of functions}

In this section, we describe auxiliary Banach spaces of functions with certain growth and decay behavior. We also provide important properties of such spaces under certain operators.

Let $U_{d}$ be an open unbounded sector with vertex at the origin in $\mathbb{C}$, bisecting direction $d \in \mathbb{R}$, and positive opening. We take $\rho>0$ and consider $D(0, \rho)=\{\tau \in \mathbb{C}:|\tau|<\rho\}$.

We fix real numbers $\beta, \mu, \delta>0, q>1$, and $\alpha \geq 0$ through the whole section. We assume that the distance from $U_{d} \cup D(0, \rho)$ to the real number $-\delta$ is strictly larger than 1 . Let $k>0$. We denote by $\bar{D}(0, \rho)$ the closure of $D(0, \rho)$.

The next definition of a Banach space of functions and subsequent properties have already been studied in previous works. Analogous spaces were treated in $[5,9]$, inspired 
by the functional spaces appearing in [17]. We refer the reader to $[7,13]$ for some of the proofs of the following results, whose statements are included for completeness.

\subsection{First family of Banach spaces of functions with $q$-exponential growth and exponential decay}

Definition 2.1 Let $q>1$. We denote by $\operatorname{Exp}_{(k, \beta, \mu, \alpha, \rho)}^{q}$ the vector space of complex-valued continuous functions $(\tau, m) \mapsto h(\tau, m)$ on $\left(U_{d} \cup \bar{D}(0, \rho)\right) \times \mathbb{R}$, holomorphic with respect to $\tau$ on $U_{d} \cup D(0, \rho)$ and such that

$$
\begin{aligned}
& \|h(\tau, m)\|_{(k, \beta, \mu, \alpha, \rho)} \\
& =\sup _{\substack{\tau \in U_{d} \cup \bar{D}(0, \rho), m \in \mathbb{R}}}(1+|m|)^{\mu} e^{\beta|m|} \exp \left(-\frac{k}{2} \frac{\log ^{2}|\tau+\delta|}{\log (q)}-\alpha \log |\tau+\delta|\right)|h(\tau, m)|
\end{aligned}
$$

is finite. The space $\left(\operatorname{Exp}_{(k, \beta, \mu, \alpha, \rho)}^{q},\|\cdot\|_{(k, \beta, \mu, \alpha, \rho)}\right)$ is a Banach space.

The proof of the following lemma is straightforward.

Lemma 2.2 Let $(\tau, m) \mapsto a(\tau, m)$ be a bounded continuous function on $\left(U_{d} \cup \bar{D}(0, \rho)\right) \times \mathbb{R}$, holomorphic with respect to $\tau$ on $U_{d} \cup D(0, \rho)$. Then

$$
\|a(\tau, m) h(\tau, m)\|_{(k, \beta, \mu, \alpha, \rho)} \leq\left(_{\tau \in U_{d} \cup \bar{D}(0, \rho), m \in \mathbb{R}}|a(\tau, m)|\right)\|h(\tau, m)\|_{(k, \beta, \mu, \alpha, \rho)}
$$

for every $h(\tau, m) \in \operatorname{Exp}_{(k, \beta, \mu, \alpha, \rho)}^{q}$.

Proposition 2.3 Let $\gamma_{1}, \gamma_{2}, \gamma_{3} \geq 0$ be such that

$$
\gamma_{1}+k \gamma_{3} \geq \gamma_{2}
$$

Let $a_{\gamma_{1}}(\tau)$ be a continuous function on $U_{d} \cup \bar{D}(0, \rho)$, holomorphic on $U_{d} \cup D(0, \rho)$, with

$$
\left|a_{\gamma_{1}}(\tau)\right| \leq \frac{1}{(1+|\tau|) \gamma_{1}}
$$

for every $\tau \in\left(U_{d} \cup \bar{D}(0, \rho)\right)$. Then there exists $C_{1}>0$, depending on $k, q, \alpha, \gamma_{1}, \gamma_{2}, \gamma_{3}$, such that

$$
\left\|a_{\gamma_{1}}(\tau) \tau^{\gamma_{2}} \sigma_{q, \tau}^{-4 \gamma_{3}} f(\tau, m)\right\|_{(k, \beta, \mu, \alpha, \rho)} \leq C_{1}\|f(\tau, m)\|_{(k, \beta, \mu, \alpha, \rho)}
$$

for every $f \in \operatorname{Exp}_{(k, \beta, \mu, \alpha, \rho)}^{q}$.

Definition 2.4 We write $E_{(\beta, \mu)}$ for the vector space of continuous functions $h: \mathbb{R} \rightarrow \mathbb{C}$ such that

$$
\|h(m)\|_{(\beta, \mu)}=\sup _{m \in \mathbb{R}}(1+|m|)^{\mu} \exp (\beta|m|)|h(m)|<\infty .
$$

Then $\left(E_{(\beta, \mu)},\|\cdot\|_{(\beta, \mu)}\right)$ is a Banach space. 
The Banach space $\left(E_{(\beta, \mu)},\|\cdot\|_{(\beta, \mu)}\right)$ can be endowed with the structure of a Banach algebra with the following noncommutative product (see Proposition 2 in [13] for further details).

Proposition 2.5 Let $Q(X), R(X) \in \mathbb{C}[X]$ be polynomials such that

$$
\operatorname{deg}(R) \geq \operatorname{deg}(Q), \quad R(\operatorname{im}) \neq 0,
$$

for all $m \in \mathbb{R}$. Let $m \mapsto b(m)$ be a continuous function in $\mathbb{R}$ such that

$$
|b(m)| \leq 1 /|R(i m)|, \quad m \in \mathbb{R}
$$

Assume that $\mu>\operatorname{deg}(Q)+1$. Then there exists a constant $C_{2}>0$ (depending on $Q(X), R(X)$, $\mu$ ) such that

$$
\left\|b(m) \int_{-\infty}^{+\infty} f\left(m-m_{1}\right) Q\left(i m_{1}\right) g\left(m_{1}\right) d m_{1}\right\|_{(\beta, \mu)} \leq C_{2}\|f(m)\|_{(\beta, \mu)}\|g(m)\|_{(\beta, \mu)}
$$

for every $f(m), g(m) \in E_{(\beta, \mu)}$. We further adopt the notation

$$
f(m) \star^{Q} g(m):=\int_{-\infty}^{+\infty} f\left(m-m_{1}\right) Q\left(i m_{1}\right) g\left(m_{1}\right) d m_{1}
$$

for $m \in \mathbb{R}$, extending the classical convolution product $\star$ for $Q \equiv 1$. As a result, $\left(E_{(\beta, \mu)}\right.$, $\left.\|\cdot\|_{(\beta, \mu)}\right)$ becomes a Banach algebra for the product $\star$ b,Q defined by

$$
f(m) \star^{b, Q} g(m):=b(m) f(m) \star^{Q} g(m) .
$$

The next proposition is a slightly modified version of Proposition 3 in [13], adapted to the appearance of two different types of growth of the functions involved, which force some positive distance to the origin.

Proposition 2.6 Let $b(m), Q(X), R(X)$ be as in Proposition 2.5. We assume that $1 \leq k \leq \kappa$ is an integer. Let $c_{h}(m) \in E_{(\beta, \mu)}$ for $h \geq 0$ be such that

$$
\left\|c_{h}\right\|_{(\beta, \mu)} \leq C\left(\frac{1}{T}\right)^{h} q^{\frac{h^{2}}{2 k}\left(1-\frac{\kappa}{k}\right)}, \quad h \geq 0
$$

for some $C>0$ and $T>q^{1 /(2 k)}$. Let $\varphi_{k}(\tau, m)$ be the power series

$$
\varphi_{k}(\tau, m)=\sum_{h \geq 0} c_{h}(m) \frac{\tau^{h}}{\left(q^{1 / k}\right)^{h(h-1) / 2}} \in E_{(\beta, \mu)} \llbracket \tau \rrbracket,
$$

which, in view of (2.1), defines an entire function with respect to $\tau$ with values in $E_{(\beta, \mu)}$.

For every $f(\tau, m) \in \operatorname{Exp}_{(\kappa, \beta, \mu, \alpha, \rho)}^{q}$, we define a q-analog of the convolution of order $k$ of $\varphi_{k}(\tau, m)$ and $f(\tau, m)$ as

$$
\varphi_{k}(\tau, m) \star_{q ; 1 / k}^{Q} f(\tau, m):=\sum_{h \geq 0} \frac{\tau^{h}}{\left(q^{1 / k}\right)^{h(h-1) / 2}} c_{h}(m) \star^{Q}\left(\sigma_{q, \tau}^{-\frac{h}{k}} f\right)(\tau, m) .
$$


Then the function $b(m) \varphi_{k}(\tau, m) \star_{q ; 1 / k}^{Q} f(\tau, m)$ belongs to $\operatorname{Exp}_{(\kappa, \beta, \mu, \alpha, \rho)}^{q}$, and there exists $C_{3}>0$, depending on $\mu, q, \alpha, k, \kappa, Q(X), R(X), \delta, T$, such that

$$
\left\|b(m) \varphi_{k}(\tau, m) \star_{q ; 1 / k}^{Q} f(\tau, m)\right\|_{(\kappa, \beta, \mu, \alpha, \rho)} \leq C_{3} C\|f(\tau, m)\|_{(\kappa, \beta, \mu, \alpha, \rho)} .
$$

Proof Let $f(\tau, m) \in \operatorname{Exp}_{(\kappa, \beta, \mu, \alpha, \rho)}^{q}$. From the definition of the norm $\|\cdot\|_{(\kappa, \beta, \mu, \alpha, \rho)}$ we know that

$$
\begin{aligned}
& \left\|b(m) \varphi_{k}(\tau, m) \star_{q ; 1 / k}^{Q} f(\tau, m)\right\|_{(\kappa, \beta, \mu, \alpha, \rho)} \\
& =\sup _{\tau \in U_{d} \cup \bar{D}(0, \rho), m \in \mathbb{R}}(1+|m|)^{\mu} e^{\beta|m|} \exp \left(-\frac{\kappa}{2} \frac{\log ^{2}(|\tau+\delta|)}{\log (q)}-\alpha \log (|\tau+\delta|)\right)|b(m)| \\
& \quad \times\left|\sum_{h \geq 0} \frac{\tau^{h}}{\left(q^{1 / k}\right)^{\frac{h(h-1)}{2}}} \int_{-\infty}^{+\infty} c_{h}\left(m-m_{1}\right) Q\left(i m_{1}\right) f\left(\frac{\tau}{q^{h / k}}, m_{1}\right) d m_{1}\right| \\
& =\sup _{\tau \in U_{d} \cup \bar{D}(0, \rho), m \in \mathbb{R}} L(\tau, m) .
\end{aligned}
$$

We first give upper estimates for

$$
\sup _{\tau \in \bar{D}(0, \rho), m \in \mathbb{R}} L(\tau, m)
$$

By construction there exist two constants $\mathfrak{Q}, \mathfrak{R}>0$ such that

$$
\left|Q\left(i m_{1}\right)\right| \leq \mathfrak{Q}\left(1+\left|m_{1}\right|\right)^{\operatorname{deg}(Q)} \text { and }|R(i m)| \geq \mathfrak{R}(1+|m|)^{\operatorname{deg}(R)}
$$

for all $m \in \mathbb{R}$. Using (2.2), from Lemma 4 in [11] (see also Lemma 2.2 in [1]) we get a constant $\tilde{C}_{2}>0$ such that

$$
\begin{aligned}
& (1+|m|)^{\mu}|b(m)| \int_{-\infty}^{+\infty} \frac{\left|Q\left(i m_{1}\right)\right|}{\left(1+\left|m-m_{1}\right|\right)^{\mu}\left(1+\left|m_{1}\right|\right)^{\mu}} d m_{1} \\
& \leq \sup _{m \in \mathbb{R}} \frac{\mathfrak{Q}}{\Re}(1+|m|)^{\mu-\operatorname{deg}(R)} \times \int_{-\infty}^{+\infty} \frac{1}{\left(1+\left|m-m_{1}\right|\right)^{\mu}\left(1+\left|m_{1}\right|\right)^{\mu-\operatorname{deg}(Q)}} d m_{1} \\
& \leq \tilde{C}_{2},
\end{aligned}
$$

provided that $\mu>\operatorname{deg}(Q)+1$. From the definition of $\|f\|_{(\kappa, \beta, \mu, \alpha, \rho)}$ and $\left\|c_{h}\right\|_{(\beta, \mu)}$ for all $h \geq 0$, (2.2), and (2.3) we get that for all $\tau \in \bar{D}(0, \rho)$ and $m \in \mathbb{R}, L(\tau, m)$ is upper bounded by

$$
\begin{aligned}
& (1+|m|)^{\mu} e^{\beta|m|} \exp \left(-\frac{\kappa}{2} \frac{\log ^{2}(|\tau+\delta|)}{\log (q)}-\alpha \log (|\tau+\delta|)\right)|b(m)| \\
& \quad \times \sum_{h \geq 0} \frac{|\tau|^{h}}{\left(q^{1 / k}\right)^{h(h-1) / 2}} \int_{-\infty}^{+\infty}\left\|c_{h}\right\|_{(\beta, \mu)} \frac{1}{\left(1+\left|m-m_{1}\right|\right)^{\mu}} e^{-\beta\left|m-m_{1}\right|}\left|Q\left(i m_{1}\right)\right| \\
& \quad \times \frac{1}{\left(1+\left|m_{1}\right|\right)^{\mu}} e^{-\beta\left|m_{1}\right|} \\
& \quad \times \exp \left(\frac{\kappa}{2} \frac{\log ^{2}\left(\left|\tau / q^{h / k}+\delta\right|\right)}{\log (q)}+\alpha \log \left(\left|\tau / q^{h / k}+\delta\right|\right)\right) d m_{1}\|f(\tau, m)\|_{(\kappa, \beta, \mu, \alpha, \rho)}
\end{aligned}
$$




$$
\begin{aligned}
\leq & \tilde{C}_{2} \exp \left(-\frac{\kappa}{2} \frac{\log ^{2}(|\tau+\delta|)}{\log (q)}-\alpha \log (|\tau+\delta|)\right) \sum_{h \geq 0} \frac{|\tau|^{h}}{\left(q^{1 / k}\right)^{h(h-1) / 2}}\left\|c_{h}\right\|_{(\beta, \mu)} \\
& \times \exp \left(\frac{\kappa}{2} \frac{\log ^{2}\left(\left|\tau / q^{h / k}+\delta\right|\right)}{\log (q)}+\alpha \log \left(\left|\tau / q^{h / k}+\delta\right|\right)\right)\|f(\tau, m)\|_{(\kappa, \beta, \mu, \alpha, \rho)} \\
\leq & \hat{C}_{2} \sum_{h \geq 0} \frac{\rho^{h}}{\left(q^{1 / k}\right)^{h(h-1) / 2}}\left\|c_{h}\right\|_{(\beta, \mu)}\|f(\tau, m)\|_{(\kappa, \beta, \mu, \alpha, \rho)}
\end{aligned}
$$

with $\hat{C}_{2}=\tilde{C}_{2} \exp \left(\frac{\kappa}{2} \frac{\log ^{2}(\rho+\delta)}{\log (q)}+\alpha \log (\rho+\delta)\right)$. Assumption (2.1) on $\left\|c_{h}\right\|_{(\beta, \mu)}$ allows us to conclude the result when restricting the domain on the variable $\tau$ to the subset $\bar{D}(0, \rho)$.

Let $\widetilde{U}_{d}$ be the complement of $\bar{D}(0, \rho)$ in $U_{d}$. From what precedes we may take the supremum over $\widetilde{U}_{d}$ instead of $U_{d} \cup \bar{D}(0, \rho)$.

By inserting terms that correspond to the $\|\cdot\|_{(\beta, \mu)}$ norm of $c_{h}(m)$ and to the $\|\cdot\|_{(\kappa, \beta, \mu, \alpha, \rho)}$ norm of $f\left(\tau / q^{h / k}, m\right)$ there exists $\tilde{C}_{1}>0$ such that we can give the bound estimates

$$
\begin{aligned}
& \sup _{\tau \in \tilde{U}_{d}, m \in \mathbb{R}} L(\tau, m) \\
& \leq \sup _{\tau \in \widetilde{U}_{d}, m \in \mathbb{R}}(1+|m|)^{\mu} e^{\beta|m|} \exp \left(-\frac{\kappa}{2} \frac{\log ^{2}(|\tau+\delta|)}{\log (q)}-\alpha \log (|\tau+\delta|)\right)|b(m)| \\
& \quad \times \sum_{h \geq 0} \int_{-\infty}^{+\infty}\left(\left(1+\left|m-m_{1}\right|\right)^{\mu} e^{\beta\left|m-m_{1}\right|} \frac{\left|c_{h}\left(m-m_{1}\right)\right|}{\left.\left(q^{1 / k}\right)^{h(h-1) / 2}|\tau|^{h}\right)}\right. \\
& \quad \times\left(\left|f\left(\frac{\tau}{q^{h / k}}, m_{1}\right)\right|\left(1+\left|m_{1}\right|\right)^{\mu} e^{\beta\left|m_{1}\right|}\right. \\
& \left.\quad \times \exp \left(-\frac{\kappa}{2} \frac{\log ^{2}\left(\left|\tau / q^{h / k}+\delta\right|\right)}{\log (q)}-\alpha \log \left(\left|\tau / q^{h / k}+\delta\right|\right)\right)\right) \\
& \quad \times\left(\frac{e^{-\beta\left|m-m_{1}\right|}}{\left(1+\left|m-m_{1}\right|\right)^{\mu}} \frac{\left|Q\left(i m_{1}\right)\right|}{\left(1+\left|m_{1}\right|\right)^{\mu}} e^{-\beta\left|m_{1}\right|}\right. \\
& \left.\quad \times \exp \left(\frac{\kappa}{2} \frac{\log ^{2}\left(\left|\tau / q^{h / k}+\delta\right|\right)}{\log (q)}+\alpha \log \left(\left|\tau / q^{h / k}+\delta\right|\right)\right)\right) d m_{1} .
\end{aligned}
$$

By means of the triangular inequality $|m| \leq\left|m-m_{1}\right|+\left|m_{1}\right|$ we deduce that

$$
\sup _{\tau \in \tilde{U}_{d}, m \in \mathbb{R}} L(\tau, m) \leq \hat{C}\|f(\tau, m)\|_{(\kappa, \beta, \mu, \alpha, \rho)},
$$

where

$$
\begin{aligned}
\hat{C}= & \sup _{\tau \in \widetilde{U}_{d}, m \in \mathbb{R}}(1+|m|)^{\mu} \exp \left(-\frac{\kappa}{2} \frac{\log ^{2}(|\tau+\delta|)}{\log (q)}-\alpha \log (|\tau+\delta|)\right)|b(m)| \\
& \times \sum_{h \geq 0}\left\|c_{h}\right\|_{(\beta, \mu)} \frac{|\tau|^{h}}{\left(q^{1 / k}\right)^{h(h-1) / 2}} \int_{-\infty}^{+\infty} \frac{\left|Q\left(i m_{1}\right)\right|}{\left(1+\left|m-m_{1}\right|\right)^{\mu}\left(1+\left|m_{1}\right|\right)^{\mu}} d m_{1} \\
& \times \exp \left(\frac{\kappa}{2} \frac{\log ^{2}\left(\left|\tau / q^{h / k}+\delta\right|\right)}{\log (q)}+\alpha \log \left(\left|\tau / q^{h / k}+\delta\right|\right)\right) .
\end{aligned}
$$


Again, we can also apply (2.3) at this point. On the other hand, we can provide upper estimates on the expression

$$
\begin{aligned}
& \exp \left(\frac{\kappa}{2 \log (q)}\left(\log ^{2}\left(\left|\frac{\tau}{q^{h / k}}+\delta\right|\right)-\log ^{2}\left(\left|\frac{\tau}{q^{h / k}}\right|\right)\right)\right) \\
& \quad \times \exp \left(\frac{\kappa}{2 \log (q)}\left(\log ^{2}\left(\left|\frac{\tau}{q^{h / k}}\right|\right)-\log ^{2}(|\tau|)\right)\right) \\
& \quad \times \exp \left(\frac{\kappa}{2 \log (q)}\left(\log ^{2}(|\tau|)-\log ^{2}(|\tau+\delta|)\right)\right) \\
& \quad \times \exp \left(\alpha \log \left(\left|\frac{\tau}{q^{h / k}}\right|\right)-\alpha \log (|\tau|)\right) \\
& \quad \times \exp \left(\alpha \log \left(\left|\frac{\tau}{q^{h / k}}+\delta\right|\right)-\alpha \log \left(\left|\frac{\tau}{q^{h / k}}\right|\right)\right) \\
& \quad \times \exp (\alpha \log (|\tau|)-\alpha \log (|\tau+\delta|))
\end{aligned}
$$

as follows. The proof of Proposition 3 in [13] can be applied to the second and fourth lines of (2.6), which yield

$$
\exp \left(\frac{\kappa}{2 \log (q)}\left(\log ^{2}\left(\left|\frac{\tau}{q^{h / k}}\right|\right)-\log ^{2}(|\tau|)\right)\right)=q^{\frac{h^{2} \kappa}{2 k^{2}}}|\tau|^{-\frac{h \kappa}{k}}
$$

and

$$
\exp \left(\alpha \log \left(\left|\frac{\tau}{q^{h / k}}\right|\right)-\alpha \log (|\tau|)\right)=q^{-\frac{\alpha h}{k}}
$$

respectively. It is straightforward to check that the expression in the fifth line of (2.6) is upper bounded by

$$
C_{31}\left(q^{h / k}\right)^{\alpha}
$$

for some positive constant $C_{31}$.

We give upper bounds for the first line in (2.6). In the case that $\left|\tau / q^{h / k}\right| \leq 1$, this expression is upper bounded by a constant that depends neither on $\tau$ nor on $h$. Otherwise, we have

$$
\begin{aligned}
& \exp \left(\frac{\kappa}{2 \log (q)}\left(\log ^{2}\left(\left|\frac{\tau}{q^{h / k}}+\delta\right|\right)-\log ^{2}\left(\left|\frac{\tau}{q^{h / k}}\right|\right)\right)\right) \\
& \quad \leq \exp \left(\frac{\kappa}{2 \log (q)}\left(\log ^{2}\left(\left|\frac{\tau}{q^{h / k}}\right|+\delta\right)-\log ^{2}\left(\left|\frac{\tau}{q^{h / k}}\right|\right)\right)\right) \\
& \quad \leq \sup _{x>1} \exp \left(\frac{\kappa}{2 \log (q)}\left(\log ^{2}(x+\delta)\right)-\log ^{2}(x)\right) \leq C_{32}
\end{aligned}
$$

for some $C_{32}>0$. We finally provide upper bounds on the third line of (2.6) taking into account that

$$
\log ^{2}|\tau|-\log ^{2}|\tau+\delta|=-\log ^{2}\left|1+\frac{\delta}{\tau}\right|-2 \log |\tau| \log \left|1+\frac{\delta}{\tau}\right| \leq C_{33}, \quad \tau \in \widetilde{U}_{d},
$$


for some $C_{33}>0$. From (2.5), (2.3), (2.6), (2.7), (2.8), (2.9), (2.10), and (2.11) we derive the existence of $\tilde{C}_{31}, \tilde{C}_{32}>0$ such that

$$
\begin{aligned}
\hat{C} & \leq \tilde{C}_{31} \sum_{h \geq 0}\left\|c_{h}\right\|_{(\beta, \mu)}\left(\sup _{\tau \in \tilde{U}_{d}}|\tau|^{h\left(1-\frac{\kappa}{k}\right)}\right) q^{\frac{h}{2 k}+\frac{h^{2}}{2 k}\left(\frac{\kappa}{k}-1\right)} \\
& \leq \tilde{C}_{32} C \sum_{h \geq 0}\left(\frac{q^{1 /(2 k)}}{T}\right)^{h} \leq C_{3} C
\end{aligned}
$$

which yields the result by choosing $T>q^{1 /(2 k)}$.

Remark Observe that condition (2.1) on the coefficients $c_{h}$ is always satisfied in the case where only a finite number of $c_{h}$ is not identically zero, that is, $\varphi_{k} \in E_{(\beta, \mu)}[\tau]$.

\subsection{Second family of Banach spaces of functions with $q$-exponential growth and exponential decay}

The second family of auxiliary Banach spaces has already been studied in previous works, such as $[7,13]$. We refer to these references for the proofs of the related results.

Let $S_{d}$ be an infinite sector of bisecting direction $d$, and let $v \in \mathbb{R}$.

Definition 2.7 We write $\operatorname{Exp}_{(k, \beta, \mu, v)}^{q}$ for the vector space of continuous functions $(\tau, m) \mapsto$ $h(\tau, m)$ on $S_{d} \times \mathbb{R}$, holomorphic with respect to $\tau$ on $S_{d}$, such that

$$
\|h(\tau, m)\|_{(k, \beta, \mu, \nu)}=\sup _{\tau \in S_{d}, m \in \mathbb{R}}(1+|m|)^{\mu} e^{\beta|m|} \exp \left(-\frac{k \log ^{2}|\tau|}{2 \log (q)}-v \log |\tau|\right)|h(\tau, m)|
$$

is finite. Then $\left(\operatorname{Exp}_{(k, \beta, \mu, v)}^{q},\|\cdot\|_{(k, \beta, \mu, v)}\right)$ is a Banach space.

Remark 2.8 Let $0 \leq \kappa_{1} \leq \kappa_{2}$. For every $f \in \operatorname{Exp}_{\left(\kappa_{1}, \beta, \mu, v\right)}^{q}$, we have that $f \in \operatorname{Exp}_{\left(\kappa_{2}, \beta, \mu, v\right)}^{q}$ and

$$
\|f(\tau, m)\|_{\left(\kappa_{2}, \beta, \mu, \nu\right)} \leq\|f(\tau, m)\|_{\left(\kappa_{1}, \beta, \mu, \nu\right)} .
$$

The proof of the following lemma is a straightforward consequence of the definition.

Lemma 2.9 Let $a(\tau, m)$ be a bounded continuous function on $S_{d} \times \mathbb{R}$, holomorphic on $S_{d}$ with respect to $\tau$. Then

$$
\|a(\tau, m) f(\tau, m)\|_{(k, \beta, \mu, \nu)} \leq \sup _{\tau \in S_{d}, m \in \mathbb{R}}|a(\tau, m)|\|f(\tau, m)\|_{(k, \beta, \mu, v)}
$$

for every $f(\tau, m) \in \operatorname{Exp}_{(k, \beta, \mu, \nu)}^{q}$.

Proposition 2.10 Let $\gamma_{1}, \gamma_{2} \geq 0$ and $\gamma_{3} \in \mathbb{R}$ be such that

$$
\gamma_{1}+k \gamma_{3} \geq \gamma_{2}
$$

Let $a_{\gamma_{1}}(\tau)$ be holomorphic on $S_{d}$ with

$$
\left|a_{\gamma_{1}}(\tau)\right| \leq \frac{1}{(1+|\tau|)^{\gamma_{1}}}, \quad \tau \in S_{d}
$$


Then there exists $C_{4}>0$, depending on $k, q, v, \gamma_{1}, \gamma_{2}, \gamma_{3}$, such that

$$
\left\|a_{\gamma_{1}}(\tau) \tau^{\gamma_{2}} \sigma_{q, \tau}^{-\gamma_{3}} f(\tau, m)\right\|_{(k, \beta, \mu, \nu)} \leq C_{4}\|f(\tau, m)\|_{(k, \beta, \mu, \nu)}
$$

for every $f \in \operatorname{Exp}_{(k, \beta, \mu, v)}^{q}$.

Proof For every $f \in \operatorname{Exp}_{(k, \beta, \mu, v)}^{q}$, we have

$$
\begin{aligned}
& \left\|a_{\gamma_{1}}(\tau) \tau^{\gamma_{2}} \sigma_{q, \tau}^{-\gamma_{3}} f(\tau, m)\right\|_{(k, \beta, \mu, v)} \\
& \leq \sup _{\tau \in S_{d}, m \in \mathbb{R}}(1+|m|)^{\mu} e^{\beta|m|} \exp \left(-\frac{k \log ^{2}|\tau|}{2 \log (q)}-v \log |\tau|\right) \frac{|\tau|^{\gamma_{2}}}{(1+|\tau|)^{\gamma_{1}}}\left|f\left(\tau / q^{\gamma_{3}}, m\right)\right| \\
& \quad \times \exp \left(-\frac{k \log ^{2}\left|\tau / q^{\gamma_{3}}\right|}{2 \log (q)}-v \log \left|\tau / q^{\gamma_{3}}\right|\right) \exp \left(\frac{k \log ^{2}\left|\tau / q^{\gamma_{3}}\right|}{2 \log (q)}+v \log \left|\tau / q^{\gamma_{3}}\right|\right) \\
& \leq \sup _{\tau \in S_{d}} q^{\frac{k \gamma_{3}^{2}-2 v \gamma_{3}}{2}} \frac{|\tau|^{-k \gamma_{3}+\gamma_{2}}}{(1+|\tau|)^{\gamma_{1}}}\|f(\tau, m)\|_{(k, \beta, \mu, v)} .
\end{aligned}
$$

The result follows from condition (2.12).

Following the same lines of arguments as in Proposition 2.6, we deduce the next proposition.

Proposition 2.11 Let $b(m), Q(X), R(X), c_{h}$ for $h \geq 0$, and let $\varphi_{k}(\tau, m)$ be chosen as in Proposition 2.6. For every $f(\tau, m) \in \operatorname{Exp}_{(k, \beta, \mu, \nu)}^{q}$, we have that $b(m) \varphi_{k}(\tau, m) \star_{q ; 1 / k}^{Q} f(\tau, m)$ belongs to $\operatorname{Exp}_{(k, \beta, \mu, v)}^{q}$ and there exists $C_{4}>0$, depending on $\mu, q, \nu, k, Q(X), R(X)$, such that

$$
\left\|b(m) \varphi_{k}(\tau, m) \star_{q ; 1 / k}^{Q} f(\tau, m)\right\|_{(k, \beta, \mu, \nu)} \leq C C_{4}\|f(\tau, m)\|_{(k, \beta, \mu, \nu)} .
$$

\section{Formal and analytic operators involved in the study of the problem}

We give the main properties of some formal and analytic transformations for completeness. In this section, $\mathbb{E}$ stands for a complex Banach space.

The definition and the main properties of the $q$-analogs of Borel and Laplace transforms in several different orders can be found in $[3,17]$. The proofs of the following results can be found in [13].

Let $q>1$ be a real number, and let $k \geq 1$ be a rational number.

Definition 3.1 For every $\hat{a}(T)=\sum_{n \geq 0} a_{n} T^{n} \in \mathbb{E} \llbracket T \rrbracket$, we define the formal $q$-Borel transform of order $k$ of $\hat{a}(T)$ by

$$
\hat{\mathcal{B}}_{q ; 1 / k}(\hat{a}(T))(\tau)=\sum_{n \geq 0} a_{n} \frac{\tau^{n}}{\left(q^{1 / k}\right)^{n(n-1) / 2}} \in \mathbb{E} \llbracket \tau \rrbracket .
$$

Proposition 3.2 Let $\sigma \in \mathbb{N}$ and $j \in \mathbb{Q}$. Then

$$
\hat{\mathcal{B}}_{q ; 1 / k}\left(T^{\sigma} \sigma_{q}^{j} \hat{a}(T)\right)(\tau)=\frac{\tau^{\sigma}}{\left(q^{1 / k}\right)^{\sigma(\sigma-1) / 2}} \sigma_{q}^{j-\frac{\sigma}{k}}\left(\hat{\mathcal{B}}_{q ; 1 / k}(\hat{a}(T))(\tau)\right)
$$

for every $\hat{a}(T) \in \mathbb{E} \llbracket T \rrbracket$. 
The $q$-analog of Laplace transform as it is shown was developed in [2]. The associated kernel of such a transform is the Jacobi theta function of order $k$ defined by

$$
\Theta_{q^{1 / k}}(x)=\sum_{n \in \mathbb{Z}} q^{-\frac{n(n-1)}{2 k}} x^{n}
$$

which turns out to be a holomorphic function in $\mathbb{C}^{\star}$. It also turns out to be a solution of the $q$-difference equation

$$
\Theta_{q^{1 / k}}\left(q^{\frac{m}{k}} x\right)=q^{\frac{m(m+1)}{2 k}} x^{m} \Theta_{q^{1 / k}}(x)
$$

for every $m \in \mathbb{Z}$, valid for all $x \in \mathbb{C}^{\star}$. As a matter of fact, the Jacobi theta function of order $k$ is a function of $q$-Gevrey decrease of order $k$ at infinity in the sense that for every $\tilde{\delta}>0$, there exists $C_{q, k}>0$, not depending on $\tilde{\delta}$, such that

$$
\left|\Theta_{q^{1 / k}}(x)\right| \geq C_{q, k} \tilde{\delta} \exp \left(\frac{k}{2} \frac{\log ^{2}|x|}{\log (q)}\right)|x|^{1 / 2}
$$

for $x \in \mathbb{C}^{\star}$ under the condition that $\left|1+x q^{\frac{m}{k}}\right|>\tilde{\delta}$ for every $m \in \mathbb{Z}$.

Definition 3.3 Let $\rho>0$, and let $U_{d}$ be an unbounded sector with vertex at 0 and bisecting direction $d \in \mathbb{R}$. Let $f: D(0, \rho) \cup U_{d} \rightarrow \mathbb{E}$ be a holomorphic function, continuous on $\bar{D}(0, \rho)$, such that there exist $K>0$ and $\alpha \in \mathbb{R}$ with

$$
\|f(x)\|_{\mathbb{E}} \leq K \exp \left(\frac{k \log ^{2}|x|}{2 \log (q)}+\alpha \log |x|\right), \quad x \in U_{d},|x| \geq \rho,
$$

and

$$
\|f(x)\|_{\mathbb{E}} \leq K, \quad x \in \bar{D}(0, \rho)
$$

Set $\pi_{q^{1 / k}}=\frac{\log (q)}{k} \prod_{n \geq 0}\left(1-\frac{1}{q^{(n+1) / k}}\right)^{-1}$. We define the $q$-Laplace transform of order $k$ of $f$ along direction $d$ by

$$
\mathcal{L}_{q ; 1 / k}^{d}(f(x))(T)=\frac{1}{\pi_{q^{1 / k}}} \int_{L_{d}} \frac{f(u)}{\Theta_{q^{1 / k}}\left(\frac{u}{T}\right)} \frac{d u}{u},
$$

where $L_{d}:=\left\{t e^{i d}: t \in(0, \infty)\right\}$.

We refer the reader to Lemma 4 and Proposition 6 in [13] for the proofs of the next results. The algebraic property held by $q$-Laplace transform will allow us to commute some operators with respect to it.

Lemma 3.4 Let $\tilde{\delta}>0$. Under the hypotheses of Definition 3.3, we have that $\mathcal{L}_{q ; 1 / k}^{d}(f(x))(T)$ defines a bounded holomorphic function on $\mathcal{R}_{d, \tilde{\delta}} \cap D\left(0, r_{1}\right)$ for every $0<r_{1} \leq q^{\left(\frac{1}{2}-\alpha\right) / k} / 2$, where

$$
\mathcal{R}_{d, \tilde{\delta}}:=\left\{T \in \mathbb{C}^{\star}:\left|1+\frac{r e^{i d}}{T}\right|>\tilde{\delta}, \text { for all } r \geq 0\right\} .
$$


A different choice for $d$ modulo $2 \pi \mathbb{Z}$ would provide the same function due to the Cauchy formula.

Proposition 3.5 Let $f$ be a function that satisfies the properties in Definition 3.3, and let $\tilde{\delta}>0$. Then, for every $\sigma \geq 0$, we have

$$
T^{\sigma} \sigma_{q}^{j}\left(\mathcal{L}_{q ; 1 / k}^{d} f(x)\right)(T)=\mathcal{L}_{q ; 1 / k}^{d}\left(\frac{x^{\sigma}}{\left(q^{1 / k}\right)^{\sigma(\sigma-1) / 2}} \sigma_{q}^{j-\frac{\sigma}{k}} f(x)\right)(T)
$$

for every $T \in \mathcal{R}_{d, \tilde{\delta}} \cap D\left(0, r_{1}\right)$ with $0<r_{1} \leq q^{\left(\frac{1}{2}-\alpha\right) / k} / 2$.

Another operator is used through the work is the inverse Fourier transform.

Proposition 3.6 Let $f \in E_{(\beta, \mu)}$ with $\beta>0$ and $\mu>1$. The inverse Fourier transform of $f$ is defined by

$$
\mathcal{F}^{-1}(f)(x)=\frac{1}{(2 \pi)^{1 / 2}} \int_{-\infty}^{\infty} f(m) \exp (i x m) d m
$$

for $x \in \mathbb{R}$. The function $\mathcal{F}^{-1}(f)$ extends to an analytic function on the strip

$$
H_{\beta}=\{z \in \mathbb{C} /|\operatorname{Im}(z)|<\beta\} .
$$

Let $\phi(m)=\operatorname{imf}(m) \in E_{(\beta, \mu-1)}$. Then we have

$$
\partial_{z} \mathcal{F}^{-1}(f)(z)=\mathcal{F}^{-1}(\phi)(z)
$$

for all $z \in H_{\beta}$.

Let $g \in E_{(\beta, \mu)}$, and let $\psi(m)=\frac{1}{(2 \pi)^{1 / 2}} f \star g(m)$, the convolution product off and $g$, for $m \in \mathbb{R}$. From Proposition 2.5 we know that $\psi \in E_{(\beta, \mu)}$. Moreover, we have

$$
\mathcal{F}^{-1}(f)(z) \mathcal{F}^{-1}(g)(z)=\mathcal{F}^{-1}(\psi)(z)
$$

for all $z \in H_{\beta}$.

\section{Formal and analytic solutions to some auxiliary convolution initial value problems with complex parameters}

Let $1 \leq k_{1}<k_{2}$ and $D, D_{1}, D_{2} \geq 3$ be integers and define $\kappa^{-1}=k_{1}^{-1}-k_{2}^{-1}$. Observe that $\kappa>k_{1}$. Let $q>1$ be a real number. We also consider the positive integer numbers $d_{D_{1}}, d_{D_{2}}$. For every $1 \leq \ell \leq D-1$, we consider nonnegative integers $d_{\ell}, \delta_{\ell} \geq 1$ and $\Delta_{\ell} \geq 0$. We assume that

$$
\delta_{1}=1, \quad \delta_{\ell}<\delta_{\ell+1}
$$

for $1 \leq \ell \leq D-2$. We also assume that

$$
\Delta_{\ell} \geq d_{\ell}, \quad \frac{d_{D_{1}}-1}{\kappa}+\frac{d_{\ell}}{k_{2}}+1 \geq \delta_{\ell}, \quad \frac{d_{\ell}}{k_{1}}+1 \geq \delta_{\ell}, \quad \frac{d_{D_{2}}-1}{k_{2}} \geq \delta_{\ell}-1
$$


for every $1 \leq \ell \leq D-1$, and also

$$
k_{1}\left(d_{D_{2}}-1\right)>k_{2} d_{D_{1}} .
$$

Let $Q(X), R_{\ell}(X) \in \mathbb{C}[X]$ for $1 \leq \ell \leq D-1$ and $R_{D_{1}}, R_{D_{2}} \in \mathbb{C}[X]$ be such that

$$
\operatorname{deg}\left(R_{D_{2}}\right)=\operatorname{deg}\left(R_{D_{1}}\right)
$$

and

$$
\begin{aligned}
& \operatorname{deg}(Q) \geq \operatorname{deg}\left(R_{D_{j}}\right) \geq \operatorname{deg}\left(R_{\ell}\right), \quad \mu-1>\operatorname{deg}\left(R_{D_{j}}\right), \\
& Q(i m) \neq 0, \quad R_{D_{j}}(i m) \neq 0,
\end{aligned}
$$

for some $\mu>\operatorname{deg}\left(R_{D_{j}}\right)+1$ with $j=1,2$ and all $m \in \mathbb{R}, 1 \leq \ell \leq D-1$.

We consider sequences of functions $m \mapsto F_{n}(m, \epsilon)$ and $m \mapsto C_{\ell, n}(m, \epsilon)$ for $n \geq 0$ belonging to the Banach space $E_{(\beta, \mu)}$ for some $\beta>0$, depending holomorphically on $\epsilon \in D\left(0, \epsilon_{0}\right)$ for some $\epsilon_{0}>0$. Moreover, we assume that there exist $\tilde{C}_{\ell}, C_{F}, T_{0}>0$ such that

$$
\left\|C_{\ell, n}\right\|_{(\beta, \mu)} \leq \tilde{C}_{\ell}\left(\frac{1}{T_{0}}\right)^{n} q^{-\frac{n^{2} \kappa}{2 k_{1} k_{2}}}, \quad\left\|F_{n}\right\|_{(\beta, \mu)} \leq C_{F}\left(\frac{1}{T_{0}}\right)^{n}, \quad n \geq 0 .
$$

We define the formal power series in $E_{(\beta, \mu)} \llbracket T \rrbracket$

$$
\hat{C}_{\ell}(T, m, \epsilon)=\sum_{n \geq 0} C_{\ell, n}(m, \epsilon) T^{n}, \quad \hat{F}(T, m, \epsilon)=\sum_{n \geq 0} F_{n}(m, \epsilon) T^{n} .
$$

We consider the initial value problem

$$
\begin{aligned}
& Q(i m) \sigma_{q, T} U(T, m, \epsilon) \\
& =T^{d_{D_{1}}} \sigma_{q, T}^{\frac{d_{D_{1}}}{k_{1}}+1} R_{D_{1}}(i m) U(T, m, \epsilon)+T^{d_{D_{2}}} \sigma_{q, T}^{\frac{d_{D_{2}}}{k_{2}}+1} R_{D_{2}}(i m) U(T, m, \epsilon) \\
& \quad+\sum_{\ell=1}^{D-1} \epsilon^{\Delta_{\ell}-d_{\ell}} T^{d_{\ell}} \sigma_{q, T}^{\delta_{\ell}}\left(\frac{1}{(2 \pi)^{1 / 2}} \int_{-\infty}^{+\infty} \hat{C}_{\ell}\left(T, m-m_{1}, \epsilon\right) R_{\ell}\left(i m_{1}\right) U\left(T, m_{1}, \epsilon\right) d m_{1}\right) \\
& \quad+\sigma_{q, T} \hat{F}(T, m, \epsilon) .
\end{aligned}
$$

Proposition 4.1 There exists a unique formal power series

$$
\hat{U}(T, m, \epsilon)=\sum_{n \geq 0} U_{n}(m, \epsilon) T^{n}
$$

solution of $(4.7)$, where the coefficients $U_{n}(m, \epsilon)$ belong to $E_{(\beta, \mu)}$ for $\beta>0$ and $\mu>\operatorname{deg}\left(R_{D_{j}}\right)+$ $1, j \in\{1,2\}$, given above and depend holomorphically on $\epsilon \in D\left(0, \epsilon_{0}\right)$.

Proof We plug the formal power series (4.8) into equation (4.7) to obtain a recursion formula for the coefficients $U_{n}, n \geq 0$. We have

$$
\begin{aligned}
& Q(i m) U_{n}(m, \epsilon) q^{n} \\
& \quad=R_{D_{1}}(i m) U_{n-d_{D_{1}}}(m, \epsilon) q^{\left(\frac{d_{D_{1}}}{k_{1}}+1\right)\left(n-d_{D_{1}}\right)}+R_{D_{2}}(i m) U_{n-d_{D_{2}}}(m, \epsilon) q^{\left(\frac{d_{D_{2}}}{k_{2}}+1\right)\left(n-d_{D_{2}}\right)}
\end{aligned}
$$




$$
\begin{aligned}
& +\sum_{\ell=1}^{D-1} \epsilon^{\Delta_{\ell}-d_{\ell}} q^{\left(n-d_{\ell}\right) \delta_{\ell}} \\
& \times\left(\sum_{n_{1}+n_{2}=n-d_{\ell}} \frac{1}{(2 \pi)^{1 / 2}} \int_{-\infty}^{+\infty} C_{\ell, n_{1}}\left(m-m_{1}, \epsilon\right) R_{\ell}\left(i m_{1}\right) U_{n_{2}}\left(m_{1}, \epsilon\right) d m_{1}\right) \\
& +F_{n}(m, \epsilon) q^{n}
\end{aligned}
$$

for every $n \geq \max \left\{d_{D_{1}}, d_{D_{2}}, \max _{1 \leq \ell \leq D-1} d_{\ell}\right\}$. Due to $C_{\ell, n}, F_{n} \in E_{(\beta, \mu)}$ for every $n \geq 0$ and $1 \leq \ell \leq D-1$, we get $U_{n} \in E_{(\beta, \mu)}$ by recursion. We observe that Proposition 2.5 is applied in the recursion.

\subsection{Analytic solutions of a first auxiliary problem in the $q$-Borel plane}

We proceed to multiply both sides of equation (4.7) by $T^{k_{1}}$ and then apply the formal $q$-Borel transform of order $k_{1}$ with respect to $T$. Let $\varphi_{k_{1}, \ell}(\tau, m, \epsilon)$ be the formal $q$-Borel transform of order $k_{1}$ of $\hat{C}_{\ell}(T, m, \epsilon)$ with respect to $T$, and let $\Psi_{k_{1}}(\tau, m, \epsilon)$ be the formal $q$-Borel transform of order $k_{1}$ of $\hat{F}(T, m, \epsilon)$ with respect to $T$. More precisely, we have

$$
\begin{aligned}
& \varphi_{k_{1}, \ell}(\tau, m, \epsilon)=\sum_{n \geq 0} C_{\ell, n}(m, \epsilon) \frac{\tau^{n}}{\left(q^{1 / k_{1}}\right)^{n(n-1) / 2}}, \\
& \Psi_{k_{1}}(\tau, m, \epsilon)=\sum_{n \geq 0} F_{n}(m, \epsilon) \frac{\tau^{n}}{\left(q^{1 / k_{1}}\right)^{n(n-1) / 2}} .
\end{aligned}
$$

According to Lemma 5 of [13], the expression $\Psi_{k_{1}}(\tau, m, \epsilon)$ represents an entire function of $q$-exponential growth of order $k_{1}$ that belongs to the Banach space $\operatorname{Exp}_{(\kappa, \beta, \mu, \alpha, \rho)}^{q}$ since $\kappa>k_{1}$, provided that $\alpha$ satisfies $T_{0}>q^{\frac{1}{2 k_{1}}} / q^{\alpha / k_{1}}$, for any unbounded sector $U_{d}$ and any disc $D(0, \rho)$. More precisely, we have

$$
\left\|\Psi_{k_{1}}(\tau, m, \epsilon)\right\|_{(\kappa, \beta, \mu, \alpha, \rho)} \leq C_{\Psi_{k_{1}}}
$$

for some constant $C_{\Psi_{k_{1}}}>0$ and all $\epsilon \in D\left(0, \epsilon_{0}\right)$.

In view of the properties of the $q$-Borel transform of order $k_{1}$, we arrive at the equation

$$
\begin{aligned}
& Q(i m) \frac{\tau^{k_{1}}}{\left(q^{1 / k_{1}}\right)^{\frac{k_{1}\left(k_{1}-1\right)}{2}}} w_{k_{1}}(\tau, m, \epsilon) \\
& =R_{D_{1}}(i m) \frac{\tau^{d_{D_{1}}+k_{1}}}{\left(q^{1 / k_{1}} \frac{\left(d_{\left.D_{1}+k_{1}\right)\left(d_{D_{1}}+k_{1}-1\right)}\right.}{2}\right.} w_{k_{1}}(\tau, m, \epsilon) \\
& +R_{D_{2}}(i m) \frac{\tau^{d_{D_{2}}+k_{1}}}{\left(q^{1 / k_{1}}\right)^{\frac{\left(d_{D_{2}}+k_{1}\right)\left(d_{D_{2}}+k_{1}-1\right)}{2}}} \sigma_{q, \tau}^{d_{D_{2}}\left(\frac{1}{k_{2}}-\frac{1}{k_{1}}\right)} w_{k_{1}}(\tau, m, \epsilon) \\
& +\frac{\tau^{k_{1}}}{\left(q^{1 / k_{1}}\right)^{\frac{k_{1}\left(k_{1}-1\right)}{2}}} \Psi_{k_{1}}(\tau, m, \epsilon)+\sum_{\ell=1}^{D-1} \epsilon^{\Delta_{\ell}-d_{\ell}} \frac{\tau^{d_{\ell}+k_{1}}}{\left(q^{1 / k_{1}}\right)^{\left(\frac{\left(d_{\ell}+k_{1}\right)\left(d_{\ell}+k_{1}-1\right)}{2}\right.}} \sigma_{q, \tau}^{\delta_{\ell}-\frac{d_{\ell}}{k_{1}}-1} \\
& \times\left(\frac{1}{(2 \pi)^{1 / 2}} \varphi_{k_{1}, \ell}(\tau, m, \epsilon) \star_{q ; 1 / k_{1}}^{R_{\ell}} w_{k_{1}}(\tau, m, \epsilon)\right),
\end{aligned}
$$


where $w_{k_{1}}(\tau, m, \epsilon)$ stands for the formal $q$-Borel transform of order $k_{1}$ of $U(T, m, \epsilon)$ with respect to $T$. Observe the appearance only of negative powers of the dilation operator in each term in the sum of the right-hand side of the equation.

We assume that there exists an unbounded sector of bisecting direction $d_{Q, R_{D_{1}}} \in \mathbb{R}$,

$$
S_{Q, R_{D_{1}}}=\left\{z \in \mathbb{C}:|z| \geq r_{Q, R_{D_{1}}},\left|\arg (z)-d_{Q, R_{D_{1}}}\right| \leq v_{Q, R_{D_{1}}}\right\}
$$

for some $r_{Q, R_{D_{1}}}, v_{Q, R_{D_{1}}}>0$, in such a way that

$$
\frac{Q(i m)}{R_{D_{1}}(i m)} \in S_{Q, R_{D_{1}}}
$$

for every $m \in \mathbb{R}$. We factorize

$$
P_{m, 1}(\tau)=\frac{Q(i m)}{\left(q^{1 / k_{1}}\right)^{\frac{k_{1}\left(k_{1}-1\right)}{2}}}-\frac{R_{D_{1}}(i m)}{\left(q^{1 / k_{1}}\right) \frac{\left(d_{D_{1}}+k_{1}\right)\left(d_{D_{1}}+k_{1}-1\right)}{2}} \tau^{d_{D_{1}}}
$$

in the form

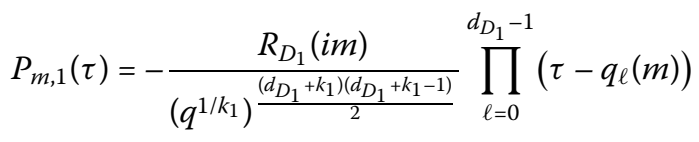

with

$$
q_{\ell}(m)=e^{\frac{2 i \pi \ell}{d_{D_{1}}}}\left(\frac{Q(i m)}{R_{D_{1}}(i m)}\right)^{1 / d_{D_{1}}} q^{\frac{d_{D_{1}}+2 k_{1}-1}{2 k_{1}}}
$$

for every $0 \leq \ell \leq d_{D_{1}}-1$. Let $U_{d}$ be an unbounded sector, and let $\rho>0$ be that the following statements hold:

(1) There exists $M_{1}>0$ such that $\left|\tau-q_{\ell}(m)\right| \geq M_{1}(1+|\tau|)$ for all $0 \leq \ell \leq d_{D_{1}}-1$, $m \in \mathbb{R}$, and $\tau \in U_{d} \cup \bar{D}(0, \rho)$. An appropriate choice of $r_{Q, R_{D_{1}}}$ and $\rho$ yields $\left|q_{\ell}(m)\right|>2 \rho$ for all $m \in \mathbb{R}$ and $0 \leq \ell \leq d_{D_{1}}-1$. In the case that $v_{Q_{,} R_{D_{1}}}$ is small enough, the set $\left\{q_{\ell}(m): m \in \mathbb{R}, 0 \leq \ell \leq d_{D_{1}}-1\right\}$ stays at a positive distance to $U_{d}$, and it can be chosen so that $1 / \tau$ has positive distance to $1 \in \mathbb{C}$ for all $\tau \in U_{d}, m \in \mathbb{R}$, and $0 \leq \ell \leq d_{D_{1}}-1$.

(2) There exists $M_{2}>0$ such that $\left|\tau-q_{\ell}(m)\right| \geq M_{2}\left|q_{\ell}(m)\right|$ for all $\ell \in\left\{0, \ldots, d_{D_{1}}-1\right\}$, $m \in \mathbb{R}$, and $\tau \in U_{d} \cup \bar{D}(0, \rho)$. This is a direct consequence of 1$)$ for some small enough $M_{2}>0$.

In order to prove the following upper estimates in (4.12), we make use of (1) for all $\ell \in\left\{0, \ldots, d_{D_{1}}-1\right\}$, except for one of them, say $\ell_{0}$, for which (2) is applied. The previous conditions yield the existence of $C_{P}>0$ such that

$$
\begin{aligned}
& \left|P_{m, 1}(\tau)\right| \\
& \quad \geq M_{1}^{d_{D_{1}}-1} M_{2} \frac{\left|R_{D_{1}}(i m)\right|(1+|\tau|)^{d_{D_{1}}-1}}{\left(q^{1 / k_{1}} \frac{\left(d_{\left.D_{1}+k_{1}\right)\left(d_{D_{1}}+k_{1}-1\right)}\right.}{2}\right.}\left(\frac{|Q(i m)|}{\left|R_{D_{1}}(i m)\right|}\right)^{1 / d_{D_{1}}} q^{\frac{d_{D_{1}}+2 k_{1}-1}{2 k_{1}}} \\
& \quad \geq C_{P}\left(r_{Q, R_{D_{1}}}\right)^{1 / d_{D_{1}}}\left|R_{D_{1}}(i m)\right|(1+|\tau|)^{d_{D_{1}}-1}
\end{aligned}
$$

for all $\tau \in U_{d} \cup \bar{D}(0, \rho)$ and $m \in \mathbb{R}$. 
The next result states the existence and uniqueness of a solution of (4.11) in the space $\operatorname{Exp}_{(\kappa, \beta, \mu, \alpha, \rho)}^{q}$, provided that its norm in that space is small enough.

Proposition 4.2 Under Assumptions (4.1), (4.2), (4.3), (4.4), and (4.5), there exist $r_{Q, R_{D_{1}}}>$ 0 , a constant $\varpi>0$, and constants $\varsigma_{\varphi}, \varsigma_{\Psi}>0$ such that if

$$
\tilde{C}_{\ell} \leq \varsigma_{\varphi}, \quad \text { and } \quad C_{\Psi_{k_{1}}} \leq \varsigma_{\Psi}
$$

for all $1 \leq \ell \leq D-1$ (see (4.6) and (4.10)), then equation (4.11) admits a unique solution $w_{k_{1}}^{d}(\tau, m, \epsilon) \in \operatorname{Exp}_{(\kappa, \beta, \mu, \alpha, \rho)}^{q}$ with $\left\|w_{k_{1}}^{d}(\tau, m, \epsilon)\right\|_{(\kappa, \beta, \mu, \alpha, \rho)} \leq \varpi$ for every $\epsilon \in D\left(0, \epsilon_{0}\right)$.

Proof Let $\epsilon \in D\left(0, \epsilon_{0}\right)$ and consider the operator $\mathcal{H}_{\epsilon}$ defined by

$$
\begin{aligned}
\mathcal{H}_{\epsilon}^{1}(w(\tau, m)):= & \frac{R_{D_{2}}(i m)}{P_{m, 1}(\tau)} \frac{\tau^{d_{D_{2}}}}{\left(q^{1 / k_{1}}\right) \frac{\left(d_{D_{2}}+k_{1}\right)\left(d_{D_{2}}+k_{1}-1\right)}{2}} \sigma_{q, \tau}^{d_{D_{2}}\left(\frac{1}{k_{2}}-\frac{1}{k_{1}}\right)} w(\tau, m) \\
& +\sum_{\ell=1}^{D-1} \epsilon^{\Delta_{\ell}-d_{\ell}} \frac{\tau^{d_{\ell}}}{P_{m, 1}(\tau)\left(q^{1 / k_{1}}\right)^{\frac{\left(d_{\ell}+k_{1}\right)\left(d_{\ell}+k_{1}-1\right)}{2}}} \sigma_{q, \tau}^{\delta_{\ell}-\frac{d_{\ell}}{k_{1}}-1} \\
& \times\left(\frac{1}{(2 \pi)^{1 / 2}} \varphi_{k_{1}, \ell}(\tau, m, \epsilon) \star_{q ; 1 / k_{1}}^{R_{\ell}} w(\tau, m)\right) \\
& +\frac{1}{P_{m, 1}(\tau)\left(q^{1 / k_{1}}\right)^{\frac{k_{1}\left(k_{1}-1\right)}{2}}} \Psi_{k_{1}}(\tau, m, \epsilon) .
\end{aligned}
$$

Note that a fixed point of $\mathcal{H}_{\epsilon}^{1}(w(\tau, m))$ will lead to a convenient solution of (4.11). To apply the fixed point theorem, we are going to prove successively two facts.

(1) We may choose $\varsigma_{\varphi}, \zeta_{\Psi}, \varpi>0$ small enough and $r_{Q, R_{D_{1}}}>0$ large enough such that

$$
\mathcal{H}_{\epsilon}^{1}(\bar{B}(0, \varpi)) \subseteq \bar{B}(0, \varpi)
$$

where $\bar{B}(0, \varpi)$ stands for the closed disc centered at 0 with radius $\varpi$ in the Banach space $\operatorname{Exp}_{(\kappa, \beta, \mu, \alpha, \rho)}^{q}$.

(2) We have

$$
\begin{gathered}
\left\|\mathcal{H}_{\epsilon}^{1}\left(w_{1}(\tau, m)\right)-\mathcal{H}_{\epsilon}^{1}\left(w_{2}(\tau, m)\right)\right\|_{(\kappa, \beta, \mu, \alpha, \rho)} \\
\leq \frac{1}{2}\left\|w_{1}(\tau, m)-w_{2}(\tau, m)\right\|_{(\kappa, \beta, \mu, \alpha, \rho)}
\end{gathered}
$$

for all $w_{1}(\tau, m), w_{2}(\tau, m) \in \bar{B}(0, \varpi)$

Proof of (4.14) We first check (4.14). Let $w(\tau, m) \in \operatorname{Exp}_{(\kappa, \beta, \mu, \alpha, \rho)}^{q}$.

By (4.2) and the definition of $\kappa$ we find that $d_{D_{1}}-1+\kappa\left(d_{\ell} / k_{1}+1-\delta_{\ell}\right) \geq d_{\ell}$ and $d_{\ell} / k_{1}+$ $1-\delta_{\ell} \geq 0$. Thus, taking into account assumptions (4.1), (4.5) and regarding (4.12) together with Propositions 2.3 and 2.6, we get

$$
\begin{aligned}
& \| \epsilon^{\Delta_{\ell}-d_{\ell}} \frac{\tau^{d_{\ell}}}{P_{m, 1}(\tau)\left(q^{1 / k_{1}}\right)^{\frac{\left(d_{\ell}+k_{1}\right)\left(d_{\ell}+k_{1}-1\right)}{2}}} \sigma_{q, \tau}^{\delta_{\ell-\frac{d_{\ell}}{k_{1}}-1}} \\
& \quad \times\left(\frac{1}{(2 \pi)^{1 / 2}} \varphi_{k_{1}, \ell}(\tau, m, \epsilon) \star_{q ; 1 / k_{1}}^{R_{\ell}} w(\tau, m)\right) \|_{(\kappa, \beta, \mu, \alpha, \rho)}
\end{aligned}
$$




$$
\leq \epsilon_{0}^{\Delta_{\ell}-d_{\ell}} \frac{C_{1} C_{3} S_{\varphi}}{\left(q^{1 / k_{1}}\right)^{\frac{\left(d_{\ell}+k_{1}\right)\left(d_{\ell}+k_{1}-1\right)}{2}} C_{P}\left(r_{Q, R_{D_{1}}}\right)^{1 / d_{D_{1}}(2 \pi)^{1 / 2}}}\|w(\tau, m)\|_{(\kappa, \beta, \mu, \alpha, \rho)} .
$$

Gathering Lemma 2.2, we get

$$
\begin{aligned}
& \left\|\frac{1}{P_{m, 1}(\tau)\left(q^{1 / k_{1}}\right)^{k_{1}\left(k_{1}-1\right) / 2}} \Psi_{k_{1}}(\tau, m, \epsilon)\right\|_{(\kappa, \beta, \mu, \alpha, \rho)} \\
& \leq \frac{1}{\left(q^{1 / k_{1}}\right)^{k_{1}\left(k_{1}-1\right) / 2} C_{P}\left(r_{Q, R_{D_{1}}}\right)^{1 / d_{D_{1}}}} \sup _{m \in \mathbb{R}} \frac{1}{\left|R_{D_{1}}(i m)\right|} \varsigma_{\Psi} .
\end{aligned}
$$

Condition (4.4) and an application of Proposition 2.3 and Lemma 2.2 yield

$$
\begin{aligned}
& \left\|\frac{R_{D_{2}}(i m)}{P_{m, 1}(\tau)} \frac{\tau^{d_{D_{2}}}}{\left(q^{\left.1 / k_{1}\right)} \frac{\left(d_{D_{2}}+k_{1}\right)\left(d_{D_{2}}+k_{1}-1\right)}{2}\right.} \sigma_{q, \tau}^{d_{D_{2}}\left(\frac{1}{k_{2}}-\frac{1}{k_{1}}\right)} w(\tau, m)\right\|_{(\kappa, \beta, \mu, \alpha, \rho)} \\
& \leq \sup _{m \in \mathbb{R}} \frac{\left|R_{D_{2}}(i m)\right|}{\left|R_{D_{1}}(i m)\right|} \frac{C_{1}}{\left(q^{1 / k_{1}}\right)^{\frac{\left(d_{D_{2}}+k_{1}\right)\left(d_{D_{2}}+k_{1}-1\right)}{2}} C_{P}\left(r_{Q, R_{D_{1}}}\right)^{1 / d_{D_{1}}}} \varpi .
\end{aligned}
$$

An appropriate choice of $r_{Q, R_{D_{1}}}>0, \varpi, \varsigma_{\Psi}, \varsigma_{\varphi}>0$ gives

$$
\begin{aligned}
& \sum_{\ell=1}^{D-1} \epsilon_{0}^{\Delta \ell-d_{\ell}} \frac{C_{3} \zeta_{\varphi} C_{1}}{\left(q^{1 / k_{1}}\right)^{\frac{\left(d_{\ell}+k_{1}\right)\left(d_{\ell}+k_{1}-1\right)}{2}} C_{P}\left(r_{Q, R_{D_{1}}}\right)^{1 / d_{D_{1}}(2 \pi)^{1 / 2}}} \varpi \\
& +\frac{1}{\left(q^{1 / k_{1}}\right)^{k_{1}\left(k_{1}-1\right) / 2} C_{P}\left(r_{Q, R_{D_{1}}}\right)^{1 / d_{D_{1}}}} \sup _{m \in \mathbb{R}} \frac{1}{\left|R_{D_{1}}(i m)\right|} \varsigma_{\Psi} \\
& +\sup _{m \in \mathbb{R}} \frac{\left|R_{D_{2}}(i m)\right|}{\left|R_{D_{1}}(i m)\right|} \frac{C_{1} \varpi}{\left(q^{1 / k_{1}}\right)^{\frac{\left(d_{D_{2}}+k_{1}\right)\left(d_{D_{2}}+k_{1}-1\right)}{2}} C_{P}\left(r_{Q, R_{D_{1}}}\right)^{1 / d_{D_{1}}}} \leq \varpi .
\end{aligned}
$$

Regarding (4.16), (4.17), (4.18), and (4.19), we obtain (4.14).

Proof of (4.15) We proceed to prove (4.15). Let $w_{1}, w_{2} \in \operatorname{Exp}_{(\kappa, \beta, \mu, \alpha, \rho)}^{q}$. We assume that $\left\|w_{\ell}(\tau, m)\right\|_{(\kappa, \beta, \mu, \alpha, \rho)} \leq \varpi, \ell=1,2$, for some $\varpi>0$. Let $E(\tau, m):=w_{1}(\tau, m)-w_{2}(\tau, m)$. On one hand, from (4.16) we have

$$
\begin{aligned}
& \left\|\frac{\epsilon^{\Delta_{\ell}-d_{\ell}} \tau^{d_{\ell}}}{P_{m, 1}(\tau)\left(q^{1 / k_{1}}\right)^{\frac{\left(d_{\ell}+k_{1}\right)\left(d_{\ell}+k_{1}-1\right)}{2}}} \sigma_{q, \tau}^{\delta_{\ell}-\frac{d_{\ell}}{k_{1}}-1}\left(\frac{1}{(2 \pi)^{1 / 2}} \varphi_{k_{1}, \ell}(\tau, m, \epsilon) \star_{q ; 1 / k_{1}}^{R_{\ell}} E(\tau, m)\right)\right\|_{(\kappa, \beta, \mu, \alpha, \rho)} \\
& \leq \epsilon_{0}^{\Delta_{\ell}-d_{\ell}} \frac{C_{3} S_{\varphi} C_{1}}{\left(q^{1 / k_{1}}\right)^{\frac{\left(d_{\ell}+k_{1}\right)\left(d_{\ell}+k_{1}-1\right)}{2}} C_{P}\left(r_{Q, R_{1}}\right)^{1 / d_{D_{1}}}(2 \pi)^{1 / 2}}\|E(\tau, m)\|_{(\kappa, \beta, \mu, \alpha, \rho)} .
\end{aligned}
$$

On the other hand, (4.18) yields

$$
\begin{aligned}
& \left\|\frac{R_{D_{2}}(i m)}{P_{m, 1}(\tau)} \frac{\tau^{d_{D_{2}}}}{\left(q^{1 / k_{1}}\right) \frac{\left(d_{D_{2}}+k_{1}\right)\left(d_{D_{2}}+k_{1}-1\right)}{2}} \sigma_{q, \tau}^{d_{D_{2}}\left(\frac{1}{k_{2}}-\frac{1}{k_{1}}\right)} E(\tau, m)\right\|_{(\kappa, \beta, \mu, \alpha, \rho)} \\
& \leq \sup _{m \in \mathbb{R}} \frac{\left|R_{D_{2}}(i m)\right|}{\left|R_{D_{1}}(i m)\right|} \frac{C_{1}}{\left(q^{1 / k_{1}}\right)^{\frac{\left(d_{D_{2}}+k_{1}\right)\left(d_{D_{2}}+k_{1}-1\right)}{2}} C_{P}\left(r_{Q, R_{D_{1}}}\right)^{1 / d_{D_{1}}}}\|E(\tau, m)\|_{(\kappa, \beta, \mu, \alpha, \rho)} .
\end{aligned}
$$


We choose $r_{Q, R_{D_{1}}}>0$ and $\zeta_{\varphi}>0$ such that

$$
\begin{aligned}
& \sum_{\ell=1}^{D-1} \epsilon_{0}^{\Delta_{\ell}-d_{\ell}} \frac{C_{3} S_{\varphi} C_{1}}{\left(q^{1 / k_{1}}\right)^{\frac{\left(d_{\ell}+k_{1}\right)\left(d_{\ell}+k_{1}-1\right)}{2}} C_{P}\left(r_{Q, R_{D_{1}}}\right)^{1 / d_{D_{1}}(2 \pi)^{1 / 2}}} \leq \frac{1}{2} . \\
& \quad+\sup _{m \in \mathbb{R}} \frac{\left|R_{D_{2}}(i m)\right|}{\left|R_{D_{1}}(i m)\right|} \frac{C_{1}}{\left(q^{1 / k_{1}}\right)^{\frac{\left(d_{D_{2}}+k_{1}\right)\left(d_{D_{2}}+k_{1}-1\right)}{2}} C_{P}\left(r_{Q, R_{D_{1}}}\right)^{1 / d_{D_{1}}}} \leq
\end{aligned}
$$

Statement (4.15) is a direct consequence of condition (4.22) applied to (4.20) and (4.21).

Let us finish the proof of the proposition. At this point, in view of (4.14) and (4.15), we can choose $\varpi>0$ such that $\bar{B}(0, \varpi) \subseteq \operatorname{Exp}_{(\kappa, \beta, \mu, \alpha, \rho)}^{q}$, which defines a complete metric space for the norm $\|\cdot\|_{(\kappa, \beta, \mu, \alpha, \rho)}$. The map $\mathcal{H}_{\epsilon}^{1}$ is contractive from $\bar{B}(0, \varpi)$ into itself. The fixed point theorem states that $\mathcal{H}_{\epsilon}^{1}$ admits a unique fixed point $w_{k_{1}}^{d}(\tau, m, \epsilon) \in \bar{B}(0, \varpi) \subseteq$ $\operatorname{Exp}_{(\kappa, \beta, \mu, \alpha, \rho)}^{q}$ for every $\epsilon \in D\left(0, \epsilon_{0}\right)$. The construction of $w_{k_{1}}^{d}(\tau, m, \epsilon)$ allows us to conclude that it turns out to be a solution of (4.11).

The next step consists of studying the solutions of a second auxiliary problem. This problem lies in a second $q$-Borel plane, and its solution would guarantee the extension with appropriate growth of the acceleration of the solution to our first auxiliary problem described in (4.11).

We set

$$
\Psi_{k_{2}}(\tau, m, \epsilon)=\sum_{n \geq 0} F_{n}(m, \epsilon) \frac{\tau^{n}}{\left(q^{1 / k_{2}}\right)^{n(n-1) / 2}},
$$

the $q$-Borel transform of order $k_{2}$ of $\hat{F}(T, m, \epsilon)$. According to the second condition of (4.6), the expression $\Psi_{k_{2}}(\tau, m, \epsilon)$ stands for an entire function of $q$-exponential growth of order $k_{2}$ that belongs to the Banach space $\operatorname{Exp}_{\left(k_{2}, \beta, \mu, \nu\right)}^{q}$, provided that $v \in \mathbb{R}$ satisfies $T_{0}>q^{\frac{1}{2 k_{2}}} / q^{v / k_{2}}$ for any unbounded sector $S_{d}$. More precisely, we have

$$
\left\|\Psi_{k_{2}}(\tau, m, \epsilon)\right\|_{\left(k_{2}, \beta, \mu, \nu\right)} \leq C_{\Psi_{k_{2}}}
$$

for some constant $C_{\Psi_{k_{2}}}>0$ and all $\epsilon \in D\left(0, \epsilon_{0}\right)$.

\subsection{Analytic solutions of a second auxiliary problem in the $q$-Borel plane}

We multiply both sides of equation (4.7) by $T^{k_{2}}$ and apply the formal $q$-Borel transform of order $k_{2}$ with respect to $T$. In view of the properties of the $q$-Borel transform, the resulting problem is determined by

$$
\begin{aligned}
& Q(i m) \frac{\tau^{k_{2}}}{\left(q^{1 / k_{2}} \frac{k_{2}\left(k_{2}-1\right)}{2}\right.} \hat{w}_{k_{2}}(\tau, m, \epsilon) \\
& =R_{D_{1}}(i m) \frac{\tau^{d_{D_{1}}+k_{2}}}{\left(q^{1 / k_{2}}\right) \frac{\left(d_{\left.D_{1}+k_{2}\right)\left(d_{\left.D_{1}+k_{2}-1\right)}\right.}^{2}\right.}{d_{D_{1}}\left(\frac{1}{k_{1}}-\frac{1}{k_{2}}\right)} \hat{w}_{k_{2}}(\tau, m, \epsilon)} \\
& \quad+R_{D_{2}}(i m) \frac{\tau^{d_{D_{2}}+k_{2}}}{\left(q^{1 / k_{2}}\right)^{\frac{\left(d_{\left.D_{2}+k_{2}\right)\left(d_{D_{2}}+k_{2}-1\right)}\right.}{2}}} \hat{w}_{k_{2}}(\tau, m, \epsilon)+\frac{\tau^{k_{2}}}{\left(q^{1 / k_{2}}\right)^{\frac{k_{2}\left(k_{2}-1\right)}{2}}} \Psi_{k_{2}}(\tau, m, \epsilon)
\end{aligned}
$$




$$
\begin{aligned}
& +\sum_{\ell=1}^{D-1} \epsilon^{\Delta_{\ell}-d_{\ell}} \frac{\tau^{d_{\ell}+k_{2}}}{\left(q^{1 / k_{2}}\right)^{\frac{\left(d_{\ell}+k_{2}\right)\left(d_{\ell}+k_{2}-1\right)}{2}}} \sigma_{q, \tau}^{\delta_{\ell}-\frac{d_{\ell}}{k_{2}}-1} \\
& \times\left(\frac{1}{(2 \pi)^{1 / 2}} \varphi_{k_{2}, \ell}(\tau, m, \epsilon) \star_{q ; 1 / k_{2}}^{R_{\ell}} \hat{w}_{k_{2}}(\tau, m, \epsilon)\right) .
\end{aligned}
$$

Here $\hat{w}_{k_{2}}(\tau, m, \epsilon), \Psi_{k_{2}}(\tau, m, \epsilon)$, and $\varphi_{k_{2}, l}(\tau, m, \epsilon)$ stand for the formal $q$-Borel transforms of order $k_{2}$ of $U(T, m, \epsilon), \hat{F}(T, m, \epsilon)$, and $\hat{C}_{l}(T, m, \epsilon)$.

We consider our second auxiliary problem

$$
\begin{aligned}
& Q(i m) \frac{\tau^{k_{2}}}{\left(q^{1 / k_{2}}\right)^{\frac{k_{2}\left(k_{2}-1\right)}{2}}} w_{k_{2}}(\tau, m, \epsilon) \\
& =R_{D_{1}}(i m) \frac{\tau^{d_{D_{1}}+k_{2}}}{\left(q^{1 / k_{2}}\right) \frac{\left(d_{\left.D_{1}+k_{2}\right)\left(d_{D_{1}}+k_{2}-1\right)}\right.}{2}} \sigma_{q, \tau}^{d_{D_{1}}\left(\frac{1}{k_{1}}-\frac{1}{k_{2}}\right)} w_{k_{2}}(\tau, m, \epsilon) \\
& +R_{D_{2}}(i m) \frac{\tau^{d_{D_{2}}+k_{2}}}{\left(q^{1 / k_{2}}\right)^{\frac{\left(d_{D_{2}}+k_{2}\right)\left(d_{D_{2}}+k_{2}-1\right)}{2}}} w_{k_{2}}(\tau, m, \epsilon)+\frac{\tau^{k_{2}}}{\left(q^{1 / k_{2}}\right)^{\frac{k_{2}\left(k_{2}-1\right)}{2}}} \Psi_{k_{2}}(\tau, m, \epsilon) \\
& +\sum_{\ell=1}^{D-1} \epsilon^{\Delta_{\ell}-d_{\ell}} \frac{\tau^{d_{\ell}+k_{2}}}{\left(q^{1 / k_{2}}\right)^{\frac{\left(d_{\ell}+k_{2}\right)\left(d_{\ell}+k_{2}-1\right)}{2}}} \sigma_{q, \tau}^{\delta_{\ell}-\frac{d_{\ell}}{k_{2}}-1} \\
& \times\left(\frac{1}{(2 \pi)^{1 / 2}} \varphi_{k_{2}, \ell}(\tau, m, \epsilon) \star_{q ; 1 / k_{2}}^{R_{\ell}} w_{k_{2}}(\tau, m, \epsilon)\right) .
\end{aligned}
$$

We assume that there exists an unbounded sector of bisecting direction $d_{Q, R_{D_{2}}} \in \mathbb{R}$,

$$
S_{Q, R_{D_{2}}}=\left\{z \in \mathbb{C}:|z| \geq r_{Q, R_{D_{2}}},\left|\arg (z)-d_{Q, R_{D_{2}}}\right| \leq v_{Q, R_{D_{2}}}\right\}
$$

for some $v_{Q, R_{D_{2}}}>0$, such that

$$
\frac{Q(i m)}{R_{D_{2}}(i m)} \in S_{Q, R_{D_{2}}}
$$

for every $m \in \mathbb{R}$. We factorize

$$
P_{m, 2}(\tau)=\frac{Q(i m)}{\left(q^{1 / k_{2}}\right)^{\frac{k_{2}\left(k_{2}-1\right)}{2}}}-\frac{R_{D_{2}}(i m)}{\left(q^{1 / k_{2}}\right)^{\frac{\left(d_{D_{2}}+k_{2}\right)\left(d_{D_{2}}+k_{2}-1\right)}{2}}} \tau^{d_{D_{2}}}
$$

in the form

$$
P_{m, 2}(\tau)=-\frac{R_{D_{2}}(i m)}{\left(q^{1 / k_{2}}\right) \frac{\left(d_{D_{2}}+k_{2}\right)\left(d_{D_{2}}+k_{2}-1\right)}{2}} \prod_{\ell=0}^{d_{D_{2}}-1}\left(\tau-q_{\ell, 2}(m)\right) .
$$

Let $S_{d}$ be an unbounded sector with small enough aperture such that:

(1) There exists $M_{12}>0$ such that $\left|\tau-q_{\ell, 2}(m)\right| \geq M_{12}(1+|\tau|)$ for all $0 \leq \ell \leq d_{D_{2}}-1$, $m \in \mathbb{R}$, and $\tau \in S_{d}$.

(2) There exists $M_{22}>0$ such that $\left|\tau-q_{\ell, 2}(m)\right| \geq M_{22}\left|q_{\ell, 2}(m)\right|$ for all $\ell \in\left\{0, \ldots, d_{D_{2}}-1\right\}, m \in \mathbb{R}$, and $\tau \in S_{d}$. 
In the following estimates, we apply (1) in the previous assumption to all indices $\ell \epsilon$ $\left\{0, \ldots, d_{D_{2}}-1\right\}$, except one of them, say $\ell_{0}$, for which we apply (2). This yields the existence of $C_{P, 2}>0$ such that

$$
\left|P_{m, 2}(\tau)\right| \geq C_{P, 2}\left(r_{Q, R_{D_{2}}}\right)^{1 / d_{D_{2}}}\left|R_{D_{2}}(i m)\right|(1+|\tau|)^{d_{D_{2}}-1}
$$

for every $\tau \in S_{d}$, and $m \in \mathbb{R}$.

Proposition 4.3 Under hypotheses (4.1), (4.2), (4.3), (4.4), (4.5), and those on the geometry of the problem, there exist $r_{Q_{,} R_{D_{2}}}, \varpi>0$ and $\varsigma_{\varphi}, \varsigma_{\Psi}>0$ under (4.13) such that for every $\epsilon \in D\left(0, \epsilon_{0}\right)$, equation (4.26) admits a unique solution $w_{k_{2}}^{d}(\tau, m, \epsilon)$ in the space $\operatorname{Exp}_{\left(k_{2}, \beta, \mu, v\right)}^{q}$ for $v \in \mathbb{R}$ and depends holomorphically with respect to $\epsilon \in D\left(0, \epsilon_{0}\right)$. Moreover, $\left\|w_{k_{2}}^{d}(\tau, m, \epsilon)\right\|_{\left(k_{2}, \beta, \mu, v\right)} \leq \varpi$.

Proof Let $\epsilon \in D\left(0, \epsilon_{0}\right)$. We consider the map $\mathcal{H}_{\epsilon}^{2}$ defined by

$$
\begin{aligned}
\mathcal{H}_{\epsilon}^{2}(w(\tau, m)):= & \frac{R_{D_{1}}(i m)}{P_{m, 2}(\tau)} \frac{\tau^{d_{D_{1}}}}{\left(q^{1 / k_{2}}\right) \frac{\left(d_{D_{1}}+k_{2}\right)\left(d_{D_{1}}+k_{2}-1\right)}{2}} \sigma_{q, \tau}^{d_{D_{1}}\left(\frac{1}{k_{1}}-\frac{1}{k_{2}}\right)} w(\tau, m) \\
& +\sum_{\ell=1}^{D-1} \epsilon^{\Delta_{\ell}-d_{\ell}} \frac{\tau^{d_{\ell}}}{P_{m, 2}(\tau)\left(q^{1 / k_{2}}\right)^{\frac{\left(d_{\ell}+k_{2}\right)\left(d_{\ell}+k_{2}-1\right)}{2}}} \sigma_{q, \tau}^{\delta_{\ell}-\frac{d_{\ell}}{k_{2}}-1} \\
& \times\left(\frac{1}{(2 \pi)^{1 / 2}} \varphi_{k_{2}, \ell}(\tau, m, \epsilon) \star_{q ; 1 / k_{2}}^{R_{\ell}} w(\tau, m)\right) \\
& +\frac{1}{P_{m, 2}(\tau)\left(q^{1 / k_{2}}\right)^{\frac{k_{2}\left(k_{2}-1\right)}{2}}} \Psi_{k_{2}}(\tau, m, \epsilon) .
\end{aligned}
$$

Note that a fixed point of $\mathcal{H}_{\epsilon}^{2}(w(\tau, m))$ will lead to a convenient solution of (4.26). To apply the fixed point theorem, we are going to prove successively two facts.

(1) We may choose $\varsigma_{\varphi}, \varsigma_{\Psi}, \varpi>0$ small enough and $r_{Q, R_{D_{2}}}>0$ large enough such that

$$
\mathcal{H}_{\epsilon}^{2}(\bar{B}(0, \varpi)) \subseteq \bar{B}(0, \varpi)
$$

where $\bar{B}(0, \varpi)$ stands for the closed disc centered at 0 with radius $\varpi$ in the Banach space $\operatorname{Exp}_{\left(k_{2}, \beta, \mu, v\right)}^{q}$.

(2) We have

$$
\begin{aligned}
& \left\|\mathcal{H}_{\epsilon}^{2}\left(w_{1}(\tau, m)\right)-\mathcal{H}_{\epsilon}^{2}\left(w_{2}(\tau, m)\right)\right\|_{\left(k_{2}, \beta, \mu, v\right)} \\
& \quad \leq \frac{1}{2}\left\|w_{1}(\tau, m)-w_{2}(\tau, m)\right\|_{\left(k_{2}, \beta, \mu, v\right)}
\end{aligned}
$$

for all $w_{1}(\tau, m), w_{2}(\tau, m) \in \bar{B}(0, \varpi)$

Proof of (4.29) We first check (4.29). Let $w(\tau, m) \in \operatorname{Exp}_{\left(k_{2}, \beta, \mu, \nu\right)}^{q}$. 
With (4.2), we find that $d_{D_{2}}-1+k_{2}\left(d_{\ell} / k_{2}+1-\delta_{\ell}\right) \geq d_{\ell}$. Taking into account assumptions (4.1), (4.4), and (4.5), regarding (4.27) together with Lemma 2.9, Proposition 2.10, and Proposition 2.11, we get

$$
\begin{aligned}
& \| \epsilon^{\Delta_{\ell}-d_{\ell}} \frac{\tau^{d_{\ell}}}{P_{m, 2}(\tau)\left(q^{1 / k_{2}}\right)^{\frac{\left(d_{\ell}+k_{2}\right)\left(d_{\ell}+k_{2}-1\right)}{2}}} \sigma_{q, \tau}^{\delta_{\ell}-\frac{d_{\ell}}{k_{2}}-1} \\
& \quad \times\left(\frac{1}{(2 \pi)^{1 / 2}} \varphi_{k_{2}, \ell}(\tau, m, \epsilon) \star_{q ; 1 / k_{2}}^{R_{\ell}} w(\tau, m)\right) \|_{\left(k_{2}, \beta, \mu, \nu\right)} \\
& \leq \epsilon_{0}^{\Delta_{\ell}-d_{\ell}} \frac{C_{4} C_{3} \zeta_{\varphi}}{\left(q^{1 / k_{2}}\right)^{\frac{\left(d_{\ell}+k_{2}\right)\left(d_{\ell}+k_{2}-1\right)}{2}} C_{P, 2}\left(r_{Q, R_{D_{2}}}\right)^{1 / d_{D_{2}}(2 \pi)^{1 / 2}}\|w(\tau, m)\|_{\left(k_{2}, \beta, \mu, \nu\right)} .} .
\end{aligned}
$$

Gathering Lemma 2.9, we get

$$
\begin{aligned}
& \left\|\frac{1}{P_{m, 2}(\tau)\left(q^{1 / k_{2}}\right)^{k_{2}\left(k_{2}-1\right) / 2}} \Psi_{k_{2}}(\tau, m, \epsilon)\right\|_{\left(k_{2}, \beta, \mu, \nu\right)} \\
& \quad \leq \frac{1}{\left(q^{1 / k_{2}}\right)^{k_{2}\left(k_{2}-1\right) / 2} C_{P, 2}\left(r_{Q, R_{D_{2}}}\right)^{1 / d_{D_{2}}}} \sup _{m \in \mathbb{R}} \frac{1}{\left|R_{D_{2}}(i m)\right|} \varsigma_{\Psi_{2}}
\end{aligned}
$$

for some $\varsigma_{\Psi_{2}}$. Observe that $\varsigma_{\Psi_{2}}$ tends to 0 as $\varsigma_{\Psi}$ does.

Condition (4.4) and an application of Proposition 2.10 and Lemma 2.9 yield

$$
\begin{aligned}
& \left\|\frac{R_{D_{1}}(i m)}{P_{m, 2}(\tau)} \frac{\tau^{d_{D_{1}}}}{\left(q^{\left.1 / k_{2}\right)} \frac{\left(d_{\left.D_{1}+k_{2}\right)\left(d_{D_{1}}+k_{2}-1\right)}\right.}{2}\right.} \sigma_{q, \tau}^{d_{D_{1}}\left(\frac{1}{k_{1}}-\frac{1}{k_{2}}\right)} w(\tau, m)\right\|_{\left(k_{2}, \beta, \mu, v\right)} \\
& \leq \sup _{m \in \mathbb{R}} \frac{\left|R_{D_{1}}(i m)\right|}{\left|R_{D_{2}}(i m)\right|} \frac{C_{4}}{\left(q^{1 / k_{2}}\right) \frac{\left(d_{D_{1}}+k_{2}\right)\left(d_{D_{1}}+k_{2}-1\right)}{2} C_{P, 2}\left(r_{Q, R_{D_{2}}}\right)^{1 / d_{D_{2}}}} \varpi .
\end{aligned}
$$

An appropriate choice of $r_{Q, R_{D_{2}}}>0, \varpi, \varsigma_{\varphi}, \varsigma_{\Psi}>0$ gives

$$
\begin{aligned}
& \sum_{\ell=1}^{D-1} \epsilon_{0}^{\Delta_{\ell}-d_{\ell}} \frac{C_{3} S_{\varphi} C_{4}}{\left(q^{1 / k_{2}}\right)^{\frac{\left(d_{\ell}+k_{2}\right)\left(d_{\ell}+k_{2}-1\right)}{2}} C_{P, 2}\left(r_{Q, R_{D_{2}}}\right)^{1 / d_{D_{2}}(2 \pi)^{1 / 2}}} \varpi \\
& +\frac{1}{\left(q^{1 / k_{2}}\right)^{k_{2}\left(k_{2}-1\right) / 2} C_{P, 2}\left(r_{Q, R_{D_{2}}}\right)^{1 / d_{D_{2}}}} \sup _{m \in \mathbb{R}} \frac{1}{\left|R_{D_{2}}(i m)\right|} \varsigma_{\Psi} \\
& +\sup _{m \in \mathbb{R}} \frac{\left|R_{D_{1}}(i m)\right|}{\left|R_{D_{2}}(i m)\right|} \frac{C_{4} \varpi}{\left(q^{1 / k_{2}}\right)^{\frac{\left(d_{D_{1}}+k_{2}\right)\left(d_{D_{1}}+k_{2}-1\right)}{2}} C_{P, 2}\left(r_{Q, R_{D_{2}}}\right)^{1 / d_{D_{2}}}} \leq \varpi .
\end{aligned}
$$

Regarding (4.31), (4.32), (4.33), and (4.34), we obtain (4.29).

Proof of (4.30) We proceed to prove (4.30). Let $w_{1}, w_{2} \in \operatorname{Exp}_{\left(k_{2}, \beta, \mu, v\right)}^{q}$. We assume that $\left\|w_{\ell}(\tau, m)\right\|_{\left(k_{2}, \beta, \mu, v\right)} \leq \varpi, \ell=1,2$, for some $\varpi>0$. Let $E(\tau, m)=w_{1}(\tau, m)-w_{2}(\tau, m)$. On 
one hand, from (4.31) we have

$$
\begin{aligned}
& \| \epsilon^{\Delta_{\ell}-d_{\ell}} \frac{\tau^{d_{\ell}}}{P_{m, 2}(\tau)\left(q^{1 / k_{2}}\right)^{\frac{\left(d_{\ell}+k_{2}\right)\left(d_{\ell}+k_{2}-1\right)}{2}}} \sigma_{q, \tau}^{\delta_{\ell}-\frac{d_{\ell}}{k_{2}}-1} \\
& \quad \times\left(\frac{1}{(2 \pi)^{1 / 2}} \varphi_{k_{2}, \ell}(\tau, m, \epsilon) \star_{q ; 1 / k_{2}}^{R_{\ell}} E(\tau, m)\right) \|_{\left(k_{2}, \beta, \mu, v\right)} \\
& \leq \epsilon_{0}^{\Delta_{\ell}-d_{\ell}} \frac{C_{3} S_{\varphi} C_{4}}{\left(q^{1 / k_{2}}\right)^{\frac{\left(d_{\ell}+k_{2}\right)\left(d_{\ell}+k_{2}-1\right)}{2}} C_{P, 2}\left(r_{Q, R_{D_{2}}}\right)^{1 / d_{D_{2}}(2 \pi)^{1 / 2}}\|E(\tau, m)\|_{\left(k_{2}, \beta, \mu, v\right)} .}
\end{aligned}
$$

On the other hand, (4.33) yields

$$
\begin{aligned}
& \left\|\frac{R_{D_{1}}(i m)}{P_{m, 2}(\tau)} \frac{\tau^{d_{D_{1}}}}{\left(q^{1 / k_{2}}\right) \frac{\left(d_{D_{1}}+k_{2}\right)\left(d_{D_{1}}+k_{2}-1\right)}{2}} \sigma_{q, \tau}^{d_{D_{1}}\left(\frac{1}{k_{1}}-\frac{1}{k_{2}}\right)} E(\tau, m)\right\|_{\left(k_{2}, \beta, \mu, v\right)} \\
& \leq \sup _{m \in \mathbb{R}} \frac{\left|R_{D_{1}}(i m)\right|}{\left|R_{D_{2}}(i m)\right|} \frac{C_{4}}{\left(q^{1 / k_{2}}\right) \frac{\left(d_{D_{1}}+k_{2}\right)\left(d_{D_{1}}+k_{2}-1\right)}{2} C_{P, 2}\left(r_{Q, R_{D_{2}}}\right)^{1 / d_{D_{2}}}}\|E(\tau, m)\|_{\left(k_{2}, \beta, \mu, v\right)} .
\end{aligned}
$$

We choose $r_{Q, R_{D_{2}}}>0, \varsigma_{\varphi}>0$ such that

$$
\begin{aligned}
& \sum_{\ell=1}^{D-1} \epsilon_{0}^{\Delta_{\ell}-d_{\ell}} \frac{C_{3} S_{\varphi} C_{4}}{\left(q^{1 / k_{2}}\right)^{\frac{\left(d_{\ell}+k_{2}\right)\left(d_{\ell}+k_{2}-1\right)}{2}} C_{P, 2}\left(r_{Q, R_{D_{2}}}\right)^{1 / d_{D_{2}}}(2 \pi)^{1 / 2}} \\
& \quad+\sup _{m \in \mathbb{R}} \frac{\left|R_{D_{1}}(i m)\right|}{\left|R_{D_{2}}(i m)\right|} \frac{1}{\left(q^{1 / k_{2}}\right)} .
\end{aligned}
$$

We conclude (4.30). Let us finish the proof of the proposition. At this point, in view of (4.29) and (4.30), we can choose $\varpi>0$ such that $\bar{B}(0, \varpi) \subseteq \operatorname{Exp}_{\left(k_{2}, \beta, \mu, v\right)}^{q}$, which defines a complete metric space for the norm $\|\cdot\|_{\left(k_{2}, \beta, \mu, v\right)}$. The map $\mathcal{H}_{\epsilon}^{2}$ is contractive from $\bar{B}(0, \varpi)$ into itself. The fixed point theorem states that $\mathcal{H}_{\epsilon}^{2}$ admits a unique fixed point $w_{k_{2}}^{d}(\tau, m, \epsilon) \epsilon$ $\bar{B}(0, \varpi) \subseteq \operatorname{Exp}_{\left(k_{2}, \beta, \mu, v\right)}^{q}$ for every $\epsilon \in D\left(0, \epsilon_{0}\right)$. The construction of $w_{k_{2}}^{d}(\tau, m, \epsilon)$ allows us to conclude that it is a solution of (4.26).

The existing link between the acceleration of $w_{k_{1}}^{d}$ and $w_{k_{2}}^{d}$ is now provided. Both functions coincide in the intersection of their domain of definition. This fact ensures the extension of the acceleration of $w_{k_{1}}^{d}$ along direction $d$ with appropriate $q$-exponential growth to apply the $q$-Laplace transform of that order to recover the analytic solution of the main problem under study.

Proposition 4.4 We consider $w_{k_{1}}^{d}(\tau, m, \epsilon)$ constructed in Proposition 4.2. The function

$$
\tau \mapsto \mathcal{L}_{q ; 1 / \kappa}^{d}\left(w_{k_{1}}^{d}(\tau, m, \epsilon)\right):=\mathcal{L}_{q ; 1 / \kappa}^{d}\left(h \mapsto w_{k_{1}}^{d}(h, m, \epsilon)\right)(\tau)
$$

defines a bounded holomorphic function in $\mathcal{R}_{d, \tilde{\delta}} \cap D\left(0, r_{1}\right)$ for $0<r_{1} \leq q^{\left(\frac{1}{2}-\alpha\right) / \kappa} / 2$. Moreover, we have

$$
\mathcal{L}_{q ; 1 / \kappa}^{d}\left(w_{k_{1}}^{d}(\tau, m, \epsilon)\right)=w_{k_{2}}^{d}(\tau, m, \epsilon), \quad(\tau, m, \epsilon) \in S_{d}^{b} \times \mathbb{R} \times D\left(0, \epsilon_{0}\right),
$$

where $S_{d}^{b}$ is a finite sector of bisecting direction $d$. 
Proof We recall from Proposition 4.2 that $w_{k_{1}}^{d} \in \operatorname{Exp}_{(\kappa, \beta, \mu, \alpha, \rho)}^{q}$. This guarantees appropriate bounds on $\tau \in U_{d}$ to apply the $q$-Laplace transform of order $\kappa$ along direction $d$. This yields that for every $\tilde{\delta}>0$, the function $\mathcal{L}_{q ; 1 / \kappa}^{d}\left(w_{k_{1}}^{d}(\tau, m, \epsilon)\right)$ defines a bounded holomorphic function in $\mathcal{R}_{d, \tilde{\delta}} \cap D\left(0, r_{1}\right)$ for $0<r_{1} \leq q^{\left(\frac{1}{2}-\alpha\right) / \kappa} / 2$.

To prove (4.35), it is sufficient to prove that $\mathcal{L}_{q ; 1 / \kappa}^{d}\left(w_{k_{1}}^{d}(\tau, m, \epsilon)\right)$ and $w_{k_{2}}^{d}$ are both solutions of some problem, with unique solution in a certain Banach space, and so they must coincide. For that purpose, we multiply both sides of equation (4.11) by $\tau^{-k_{1}}$ and take the $q$-Laplace transform of order $\kappa$ along direction $d$.

The properties of the $q$-Laplace transform yield

$$
\begin{aligned}
& \mathcal{L}_{q ; 1 / \kappa}^{d}\left(\tau^{d_{D_{1}}} w_{k_{1}}^{d}(\tau, m, \epsilon)\right)=\left(q^{1 / \kappa}\right)^{d_{D_{1}}\left(d_{D_{1}}-1\right) / 2} \tau^{d_{D_{1}}} \sigma_{q, \tau}^{\frac{d_{D_{1}}}{, \kappa}} \mathcal{L}_{q ; 1 / \kappa}^{d}\left(w_{k_{1}}^{d}\right)(\tau, m, \epsilon), \\
& \mathcal{L}_{q ; 1 / \kappa}^{d}\left(\tau^{d_{D_{2}}} \sigma_{q, \tau}^{d_{D_{2}}\left(\frac{1}{k_{2}}-\frac{1}{k_{1}}\right)} w_{k_{1}}^{d}(\tau, m, \epsilon)\right)=\left(q^{1 / \kappa}\right)^{d_{D_{2}}\left(d_{D_{2}}-1\right) / 2} \tau^{d_{D_{2}}} \mathcal{L}_{q ; 1 / \kappa}^{d}\left(w_{k_{1}}^{d}\right)(\tau, m, \epsilon),
\end{aligned}
$$

and

$$
\begin{aligned}
& \mathcal{L}_{q ; 1 / \kappa}^{d}\left(\tau^{d_{\ell}} \sigma_{q, \tau}^{\delta_{\ell}+\frac{d_{\ell}}{k_{1}}-1}\left(\frac{1}{(2 \pi)^{1 / 2}} \varphi_{k_{1}, \ell}(\tau, m, \epsilon) \star_{q ; 1 / k_{1}}^{R_{\ell}} w_{k_{1}}^{d}(\tau, m, \epsilon)\right)\right) \\
& =\left(q^{1 / \kappa}\right)^{d_{\ell}\left(d_{\ell}-1\right) / 2} \tau^{d_{\ell}} \sigma_{q, \tau}^{\delta_{\ell}-\frac{d_{\ell}}{k_{2}}-1} \mathcal{L}_{q ; 1 / \kappa}^{d}\left(\frac{1}{(2 \pi)^{1 / 2}} \varphi_{k_{1}, \ell}(\tau, m, \epsilon) \star_{q ; 1 / k_{1}}^{R_{\ell}} w_{k_{1}}^{d}(\tau, m, \epsilon)\right) .
\end{aligned}
$$

We claim that

$$
\mathcal{L}_{q ; 1 / \kappa}^{d}\left(\varphi_{k_{1}, \ell}(\tau, m, \epsilon) \star_{q ; 1 / k_{1}}^{R_{\ell}} w_{k_{1}}^{d}(\tau, m, \epsilon)\right)=\varphi_{k_{2}, \ell}(\tau, m, \epsilon) \star_{q ; 1 / k_{2}}^{R_{\ell}} \mathcal{L}_{q ; 1 / \kappa}^{d}\left(w_{k_{1}}^{d}(\tau, m, \epsilon)\right) .
$$

This is a consequence of the change of integration order in the operators involved in (4.39). This situation is different from that of (60) in the proof of Proposition 12 in [7]. Assume that the variable of integration with respect to Laplace operator is $r$. After the change of variable $\tilde{r}=r / q^{h / k_{1}}$, we reduce the study to that of $\Xi$ in the proof of Proposition 12 in [7], with $r$ replaced by $r^{1-h}$. This last argument guarantees the availability of the change of order in the integration operators involved in (4.39). We now give a proof of (4.39) under this consideration.

We have

$$
\begin{aligned}
& \mathcal{L}_{q ; 1 / \kappa}^{d}\left(\varphi_{k_{1}, \ell}(\tau, m, \epsilon) \star_{q ; 1 / k_{1}}^{R_{\ell}} w_{k_{1}}^{d}(\tau, m, \epsilon)\right) \\
& =\frac{1}{\pi_{q^{1 / \kappa}}} \int_{0}^{\infty}\left(\varphi_{k_{1}, \ell}\left(r e^{i d}, m, \epsilon\right) \star_{q ; 1 / k_{1}}^{R_{\ell}} w_{k_{1}}^{d}\left(r e^{i d}, m, \epsilon\right)\right) \frac{1}{\Theta_{q^{1 / \kappa}}\left(\frac{r e^{i d}}{\tau}\right)} \frac{d r}{r} \\
& =\frac{1}{\pi_{q^{1 / \kappa}}} \int_{0}^{\infty}\left(\sum_{n \geq 0} \frac{\left(r e^{i d}\right)^{n}}{\left(q^{1 / k_{1}}\right)^{n(n-1) / 2}} C_{\ell, n}(m, \epsilon) \star^{R_{\ell}}\left(\sigma_{q, \tau}^{-\frac{n}{k_{1}}} w_{k_{1}}^{d}\right)\left(r e^{i d}, m, \epsilon\right)\right) \frac{1}{\Theta_{q^{1 / \kappa}\left(\frac{r e^{i d}}{\tau}\right)}} \frac{d r}{r} \\
& =\frac{1}{\pi_{q^{1 / \kappa}}} \int_{0}^{\infty}\left(\sum_{n \geq 0} \frac{\left(r e^{i d}\right)^{n}}{\left(q^{1 / k_{1}}\right)^{n(n-1) / 2}}\right. \\
& \left.\quad \times \int_{-\infty}^{\infty} C_{\ell, n}\left(m-m_{1}, \epsilon\right) R_{\ell}\left(i m_{1}\right) w_{k_{1}}^{d}\left(r e^{i d} q^{-\frac{n}{k_{1}}}, m_{1}, \epsilon\right) d m_{1}\right) \frac{1}{\Theta_{q^{1 / \kappa}\left(\frac{r e^{i d}}{\tau}\right.}} \frac{d r}{r} .
\end{aligned}
$$


We make the change of variable $\tilde{r}=r / q^{n / k_{1}}$ to get that the previous expression equals

$$
\begin{aligned}
& \frac{1}{\pi_{q^{1 / \kappa}}} \int_{0}^{\infty}\left(\int_{-\infty}^{\infty} \sum_{n \geq 0} \frac{\left(\tilde{r} e^{i d}\right)^{n} q^{n^{2} / k_{1}}}{\left(q^{1 / k_{1}}\right)^{n(n-1) / 2}} C_{\ell, n}\left(m-m_{1}, \epsilon\right) R_{\ell}\left(i m_{1}\right) w_{k_{1}}^{d}\left(\tilde{r} e^{i d}, m_{1}, \epsilon\right) d m_{1}\right) \\
& \times \frac{1}{\Theta_{q^{1 / \kappa}}\left(\frac{\tilde{r} e^{i d} q^{n / k_{1}}}{\tau}\right)} \frac{d \tilde{r}}{\tilde{r}} .
\end{aligned}
$$

In view of (3.1), $k_{1}^{-1}=\kappa^{-1}+k_{2}^{-1}$, the change of order of the integrals, and the dominated convergence theorem, the previous equation equals

$$
\begin{aligned}
& =\frac{1}{\pi_{q^{1 / \kappa}}} \int_{0}^{\infty}\left(\int_{-\infty}^{\infty} \sum_{n \geq 0} \frac{\left(\tilde{r} e^{i d}\right)^{n} q^{n^{2} / k_{1}}}{\left(q^{1 / k_{1}}\right)^{n(n-1) / 2}} C_{\ell, n}\left(m-m_{1}, \epsilon\right) R_{\ell}\left(i m_{1}\right) w_{k_{1}}^{d}\left(\tilde{r} e^{i d}, m_{1}, \epsilon\right) d m_{1}\right)
\end{aligned}
$$

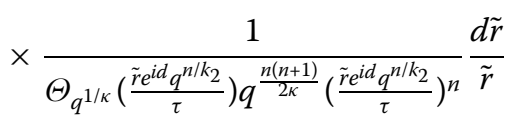

$$
\begin{aligned}
& =\frac{1}{\pi_{q^{1 / \kappa}}} \int_{0}^{\infty}\left(\int_{-\infty}^{\infty} \sum_{n \geq 0} \frac{\tau^{n} q^{n(n-1) /(2 \kappa)}}{\left(q^{1 / k_{1}}\right)^{n(n-1) / 2}} C_{\ell, n}\left(m-m_{1}, \epsilon\right) R_{\ell}\left(i m_{1}\right) w_{k_{1}}^{d}\left(\tilde{r} e^{i d}, m_{1}, \epsilon\right) d m_{1}\right) \\
& \times \frac{1}{\Theta_{q^{1 / \kappa}}\left(\frac{\tilde{r} e}{\tau} \frac{d q^{n / k_{2}}}{\tau}\right)} \frac{d \tilde{r}}{\tilde{r}} \\
& =\int_{-\infty}^{\infty}\left(\sum_{n \geq 0} \frac{\tau^{n}}{\left(q^{1 / k_{2}}\right)^{n(n-1) / 2}} C_{\ell, n}\left(m-m_{1}, \epsilon\right)\right) R_{\ell}\left(i m_{1}\right) \\
& \times\left[\frac{1}{\pi_{q^{1 / \kappa}}} \int_{0}^{\infty} \frac{w_{k_{1}}^{d}\left(\tilde{r} e^{i d}, m_{1}, \epsilon\right)}{\Theta_{q^{1 / \kappa}}\left(\frac{\tilde{r} e^{i d} q^{n / k_{2}}}{\tau}\right)} \frac{d \tilde{r}}{\tilde{r}}\right] d m_{1} \\
& =\varphi_{k_{2}, \ell}(\tau, m, \epsilon) \star_{q ; 1 / k_{2}}^{R_{\ell}} \mathcal{L}_{q ; 1 / \kappa}^{d}\left(w_{k_{1}}^{d}(\tau, m, \epsilon)\right) \text {, }
\end{aligned}
$$

from which we obtain (4.39).

On the other hand, we observe by direct computation that

$$
\mathcal{L}_{q ; 1 / \kappa}^{d}\left(\Psi_{k_{1}}(\tau, m, \epsilon)\right)=\Psi_{k_{2}}(\tau, m, \epsilon)
$$

for every $(\tau, m, \epsilon) \in\left(\mathcal{R}_{d, \tilde{\delta}} \cap D\left(0, r_{1}\right)\right) \times \mathbb{R} \times D\left(0, \epsilon_{0}\right)$.

In view of (4.36), (4.37), (4.38), (4.39), and the last formula before (4.40), we derive that

$$
\begin{aligned}
& \frac{Q(i m)}{\left(q^{1 / k_{1}}\right)^{\frac{k_{1}\left(k_{1}-1\right)}{2}}} \mathcal{L}_{q ; 1 / \kappa}^{d}\left(w_{k_{1}}^{d}\right)(\tau, m, \epsilon) \\
& =R_{D_{1}}(i m) \frac{\left(q^{1 / \kappa}\right)^{d_{D_{1}}\left(d_{D_{1}}-1\right) / 2}}{\left(q^{1 / k_{1}}\right) \frac{\left(d_{D_{1}}+k_{1}\right)\left(d_{D_{1}}+k_{1}-1\right)}{2}} \tau^{d_{D_{1}}} \sigma_{q, \tau}^{\frac{d_{D_{1}}}{q,}} \mathcal{L}_{q ; 1 / \kappa}^{d}\left(w_{k_{1}}^{d}\right)(\tau, m, \epsilon) \\
& +R_{D_{2}}(i m) \frac{\left(q^{1 / \kappa}\right)^{\frac{d_{D_{2}}\left(d_{D_{2}}-1\right)}{2}}}{\left(q^{1 / k_{1}}\right)^{\frac{\left(d_{D_{2}}+k_{1}\right)\left(d_{D_{2}}+k_{1}-1\right)}{2}}} \tau^{d_{D_{2}}} \mathcal{L}_{q ; 1 / \kappa}^{d}\left(w_{k_{1}}^{d}\right)(\tau, m, \epsilon)
\end{aligned}
$$




$$
\begin{aligned}
& +\frac{1}{\left(q^{1 / k_{1}}\right)^{\frac{k_{1}\left(k_{1}-1\right)}{2}}} \Psi_{k_{2}}(\tau, m, \epsilon)+\sum_{\ell=1}^{D-1} \epsilon^{\Delta_{\ell}-d_{\ell}} \tau^{d_{\ell}} \frac{\left(q^{1 / \kappa}\right)^{\frac{d_{\ell}\left(d_{\ell}-1\right)}{2}}}{\left(q^{1 / k_{1}} \frac{\left(d_{\ell}+k_{1}\right)\left(d_{\ell}+k_{1}-1\right)}{2}\right.} \sigma_{q, \tau}^{\delta_{\ell}-\frac{d_{\ell}}{k_{2}}-1} \\
& \times\left(\frac{1}{(2 \pi)^{1 / 2}} \varphi_{k_{2}, \ell}(\tau, m, \epsilon) \star_{q ; 1 / k_{2}}^{R_{\ell}} \mathcal{L}_{q ; 1 / \kappa}^{d}\left(w_{k_{1}}^{d}\right)(\tau, m, \epsilon)\right)
\end{aligned}
$$

for every $(\tau, m, \epsilon) \in\left(\mathcal{R}_{d, \tilde{\delta}} \cap D\left(0, r_{1}\right)\right) \times \mathbb{R} \times D\left(0, \epsilon_{0}\right)$. We multiply both sides of the previous equation by $\left(q^{1 / k_{1}}\right)^{k_{1}\left(k_{1}-1\right) / 2} /\left(q^{1 / k_{2}}\right)^{k_{2}\left(k_{2}-1\right) / 2}$. The fact that

$$
\frac{\left(q^{1 / \kappa}\right)^{\frac{\mathcal{D}(\mathcal{D}-1)}{2}}\left(q^{1 / k_{1}}\right)^{\frac{k_{1}\left(k_{1}-1\right)}{2}}}{\left(q^{1 / k_{1}}\right)^{\frac{\left(\mathcal{D}+k_{1}\right)\left(\mathcal{D}+k_{1}-1\right)}{2}}\left(q^{1 / k_{2}}\right)^{\frac{k_{2}\left(k_{2}-1\right)}{2}}}=\frac{1}{\left(q^{1 / k_{2}}\right)^{\frac{\left(\mathcal{D}+k_{2}\right)\left(\mathcal{D}+k_{2}-1\right)}{2}}}
$$

with $\mathcal{D} \in\left\{d_{D_{1}}, d_{D_{2}}, d_{\ell}\right\}$ entails that $\mathcal{L}_{q ; 1 / \kappa}^{d}\left(w_{k_{1}}^{d}(\tau, m, \epsilon)\right)$ is a solution of (4.26) in its domain of definition.

Let $S_{d}^{b}$ be a bounded sector of bisecting direction $d$ such that $S_{d}^{b} \subseteq\left(\mathcal{R}_{d, \tilde{\delta}} \cap D\left(0, r_{1}\right)\right) \cap S_{d}$, which is a nonempty set due to the assumptions on the construction of these sets. The functions $\mathcal{L}_{q ; 1 / \kappa}^{d}\left(w_{k_{1}}^{d}(\tau, m, \epsilon)\right)$ and $w_{k_{2}}^{d}(\tau, m, \epsilon)$ are continuous complex functions defined on $S_{d}^{b} \times \mathbb{R} \times D\left(0, \epsilon_{0}\right)$ and holomorphic with respect to $\tau$ (resp., $\left.\epsilon\right)$ on $S_{d}^{b}$ (resp., $D\left(0, \epsilon_{0}\right)$ ).

Let $\epsilon \in D\left(0, \epsilon_{0}\right)$ and put $\Omega=\min \{\alpha, \nu\}$. It is straightforward to check that both functions belong to the complex Banach space $H_{\left(k_{2}, \beta, \mu, \Omega\right)}$ of all continuous functions $(\tau, m) \mapsto$ $h(\tau, m)$ defined on $S_{d}^{b} \times \mathbb{R}$ and holomorphic with respect to $\tau$ in $S_{d}^{b}$ such that

$$
\|h(\tau, m)\|_{H_{\left(k_{2}, \beta, \mu, \Omega\right)}}=\sup _{\tau \in S_{d}^{b}, m \in \mathbb{R}}(1+|m|)^{\mu} e^{\beta|m|} \exp \left(-\frac{k_{2}}{2} \frac{\log ^{2}|\tau|}{\log (q)}-\Omega \log |\tau|\right)|h(\tau, m)|
$$

is finite. Then $\mathcal{L}_{q ; 1 / \kappa}^{d}\left(w_{k_{1}}^{d}(\tau, m, \epsilon)\right), w_{k_{2}}^{d}(\tau, m, \epsilon)$, and $\Psi_{k_{2}}(\tau, m, \epsilon)$ belong to $H_{\left(k_{2}, \beta, \mu, \Omega\right)}$ due to Propositions 4.2 and 4.3. As we can see in the proof of Proposition 4.3, the operator $\mathcal{H}_{\epsilon}^{2}$ defined in (4.28) has a unique fixed point in $H_{\left(k_{2}, \beta, \mu, \Omega\right)}$, provided that constants $\varsigma_{\Psi}, \varsigma_{\varphi}>0$ are small enough, for $1 \leq \ell \leq D-1$. Indeed, this fixed point is a solution of the auxiliary problem (4.26) in the disc $D(0, \varsigma)$ of $H_{\left(k_{2}, \beta, \mu, \Omega\right)}$, whilst $\mathcal{L}_{q ; 1 / \kappa}^{d}\left(w_{k_{1}}^{d}(\tau, m, \epsilon)\right), w_{k_{2}}^{d}(\tau, m, \epsilon)$ are both solutions of the same problem in the disc $D(0, \varsigma)$ of $H_{\left(k_{2}, \beta, \mu, \Omega\right)}$, so they do coincide in the domain $S_{d}^{b} \times \mathbb{R} \times D\left(0, \epsilon_{0}\right)$. Identity (4.35) follows from here.

\section{Analytic solutions to a $q$-difference-differential equation}

This section is devoted to determine in detail the main problem under study and provide an analytic solution to it. It is worth mentioning that, although the techniques developed in previous sections are essentially novel, once the tools have been implemented, the procedure of construction of the solution coincides with that explained in Sect. 5 of [7]. For completeness and a self-contained work, we describe every step of the construction in detail, whilst we have decided to pass over the proofs which can be found in [7].

Let $1 \leq k_{1}<k_{2}$. We define $1 / \kappa=1 / k_{1}-1 / k_{2}$ and take integers $D, D_{1}, D_{2}$ greater than 3 . Let $q>1$ be a real number. We also consider positive integers $d_{D_{1}}, d_{D_{2}}$, and for every $1 \leq$ $\ell \leq D-1$, we choose nonnegative integers $d_{\ell}, \delta_{\ell} \geq 1$ and $\Delta_{\ell} \geq 0$. We make the following assumptions on the previous constants.

Assumption (A) $\delta_{1}=1$ and $\delta_{\ell}<\delta_{\ell+1}$ for every $1 \leq \ell \leq D-2$. 
Assumption (B) We have

$$
\Delta_{\ell} \geq d_{\ell}, \quad \frac{d_{D_{1}}-1}{\kappa}+\frac{d_{\ell}}{k_{2}}+1 \geq \delta_{\ell}, \quad \frac{d_{\ell}}{k_{1}}+1 \geq \delta_{\ell}, \quad \frac{d_{D_{2}}-1}{k_{2}} \geq \delta_{\ell}-1,
$$

for every $1 \leq \ell \leq D-1$, and

$$
k_{1}\left(d_{D_{2}}-1\right)>k_{2} d_{D_{1}} .
$$

Let $Q, R_{D_{1}}, R_{D_{2}}$, and $R_{\ell}$ for $1 \leq \ell \leq D-1$ be polynomials with complex coefficients such that:

Assumption (C) $\operatorname{deg}\left(R_{D_{2}}\right)=\operatorname{deg}\left(R_{D_{1}}\right), \operatorname{deg}(Q) \geq \operatorname{deg}\left(R_{D_{1}}\right) \geq \operatorname{deg}\left(R_{\ell}\right)$. Moreover, we assume that $Q(i m) \neq 0$ and $R_{D_{j}}($ im $) \neq 0$ for all $m \in \mathbb{R}, 1 \leq \ell \leq D-1$.

Let $S_{Q, R_{D_{1}}}$ and $S_{Q, R_{D_{2}}}$ be unbounded sectors of bisecting directions $d_{Q, R_{D_{1}}} \in \mathbb{R}$ and $d_{Q, R_{D_{2}}} \in \mathbb{R}$, respectively, with

$$
S_{Q, R_{D_{j}}}=\left\{z \in \mathbb{C}:|z| \geq r_{Q, R_{D_{j}}},\left|\arg (z)-d_{Q, R_{D_{j}}}\right| \leq v_{Q, R_{D_{j}}}\right\}
$$

for some $v_{Q, R_{D_{j}}}>0$ and such that

$$
\frac{Q(i m)}{R_{D_{j}}(i m)} \in S_{Q, R_{D_{j}}}
$$

for every $m \in \mathbb{R}$.

Definition 5.1 Let $\varsigma \geq 2$ be an integer. A family $\left(\mathcal{E}_{p}\right)_{0 \leq p \leq s-1}$ is said to be a good covering in $\mathbb{C}^{\star}$ (in the $\epsilon$ plane) if the following hypotheses hold:

- $\mathcal{E}_{p}$ is an open sector of finite radius $\epsilon_{0}>0$ and vertex at the origin for every $0 \leq p \leq \varsigma-1$.

- $\mathcal{E}_{j} \cap \mathcal{E}_{k} \neq \emptyset$ for $0 \leq j, k \leq \varsigma-1$ if and only if $|j-k| \leq 1$ (we put $\mathcal{E}_{\varsigma}:=\mathcal{E}_{0}$ ).

- $\bigcup_{p=0}^{5-1} \mathcal{E}_{p}=\mathcal{U} \backslash\{0\}$ for some neighborhood of the origin $\mathcal{U}$.

Definition 5.2 Let $\left(\mathcal{E}_{p}\right)_{0 \leq p \leq \varsigma_{-1}}$ be a good covering. Let $\mathcal{T}$ be an open bounded sector with vertex at the origin and radius $r_{\mathcal{T}}>0$. Given $\alpha \in \mathbb{R}$ and $v \in \mathbb{R}$, we assume that

$$
\begin{aligned}
& 0<\epsilon_{0}, r_{\mathcal{T}}<1, \quad v+\frac{k_{2}}{\log (q)} \log \left(r_{\mathcal{T}}\right)<0, \\
& \alpha+\frac{\kappa}{\log (q)} \log \left(\epsilon_{0} r_{\mathcal{T}}\right)<0, \quad \epsilon_{0} r_{\mathcal{T}} \leq q^{\left(\frac{1}{2}-v\right) / k_{2}} / 2 .
\end{aligned}
$$

We consider a family of unbounded sectors $U_{\mathfrak{D}_{p}}, 0 \leq p \leq \varsigma-1$, with bisecting direction $\mathfrak{d}_{p} \in \mathbb{R}$ and a family of open domains $\mathcal{R}_{\mathfrak{d}_{p}}^{b}:=\mathcal{R}_{\mathfrak{d}_{p}, \tilde{\delta}} \cap D\left(0, \epsilon_{0} r_{\mathcal{T}}\right)$ with

$$
\mathcal{R}_{\mathfrak{d}_{p}, \tilde{\delta}}:=\left\{T \in \mathbb{C}^{\star}:\left|1+\frac{r e^{i \mathfrak{d}_{p}}}{T}\right|>\tilde{\delta} \text { for every } r \geq 0\right\}
$$

for some $\tilde{\delta}<1$. We assume that $\mathfrak{d}_{p}, 0 \leq p \leq \varsigma-1$, are chosen to satisfy the following conditions: there exist $S_{\mathfrak{o}_{p}} \cup \bar{D}(0, \rho)$ and $\rho>0$ such that 
- Conditions (1) and (2) in Sect. 4.1 hold. Observe that, under this assumption, Conditions (1) and (2) in Sect. 4.2 hold for $S_{\mathfrak{d}_{p}}$.

- For every $0 \leq p \leq \varsigma-1$, we have $\mathcal{R}_{\mathfrak{d}_{p}}^{b} \cap \mathcal{R}_{\mathfrak{d}_{p+1}}^{b} \neq \emptyset$, and for all $t \in \mathcal{T}$ and $\epsilon \in \mathcal{E}_{p}$, we have $\epsilon t \in \mathcal{R}_{\mathfrak{d}_{p}}^{b}$ (where $\mathcal{R}_{\mathfrak{d}_{5}}:=\mathcal{R}_{\mathfrak{d}_{0}}$ ).

The family $\left\{\left(\mathcal{R}_{\mathfrak{d}_{p}, \tilde{\delta}}\right)_{0 \leq p \leq \varsigma_{-1}}, D(0, \rho), \mathcal{T}\right\}$ is said to be associated with the good covering $\left(\mathcal{E}_{p}\right)_{0 \leq p \leq \varsigma-1}$.

Let $\left(\mathcal{E}_{p}\right)_{0 \leq p \leq \varsigma^{-1}}$ be a good covering, and let a family $\left\{\left(\mathcal{R}_{\mathfrak{o}_{p}, \tilde{\delta}}\right)_{0 \leq p \leq \varsigma_{-1}}, D(0, \rho), \mathcal{T}\right\}$ be associated with it. For every $0 \leq p \leq \varsigma-1$, we study the equation

$$
\begin{aligned}
& Q\left(\partial_{z}\right) \sigma_{q, t} u^{\mathfrak{d}_{p}}(t, z, \epsilon) \\
& =(\epsilon t)^{d_{D_{1}}} \sigma_{q, t}^{\frac{d_{D_{1}}}{k_{1}}+1} R_{D_{1}}\left(\partial_{z}\right) u^{\mathfrak{d}_{p}}(t, z, \epsilon)+(\epsilon t)^{d_{D_{2}}} \sigma_{q, t}^{\frac{d_{D_{2}}}{k_{2}}+1} R_{D_{2}}\left(\partial_{z}\right) u^{\mathfrak{d}_{p}}(t, z, \epsilon) \\
& \quad+\sum_{\ell=1}^{D-1} \epsilon^{\Delta_{\ell}} t^{d_{\ell}} \sigma_{q, t}^{\delta_{\ell}}\left(c_{\ell}(t, z, \epsilon) R_{\ell}\left(\partial_{z}\right) u^{\mathfrak{d}_{p}}(t, z, \epsilon)\right)+\sigma_{q, t} f(t, z, \epsilon) .
\end{aligned}
$$

The terms $c_{\ell}(t, z, \epsilon)$ are determined for every $1 \leq \ell \leq D-1$ as follows. Let $C_{\ell}(T, m, \epsilon)$ be the entire function in $T$, with coefficients in $E_{(\beta, \mu)}$ for some $\beta>0$ and $\mu \in \mathbb{R}$, given by

$$
C_{\ell}(T, m, \epsilon)=\sum_{n \geq 0} C_{\ell, n}(m, \epsilon) T^{n}
$$

such that $\mu-1 \geq \operatorname{deg}\left(R_{D_{j}}\right)$, for $j \in\{1,2\}$. Assume that this function depends holomorphically on $\epsilon \in D\left(0, \epsilon_{0}\right)$ and also that there exist $\tilde{C}_{\ell}, T_{0}>0$ such that the left-hand side of (4.6) holds for all $n \geq 0$ and $\epsilon \in D\left(0, \epsilon_{0}\right)$. We put

$$
c_{\ell}(t, z, \epsilon):=\mathcal{F}^{-1}\left(m \mapsto C_{\ell}(\epsilon t, m, \epsilon)\right)(z),
$$

which is a holomorphic bounded function on $\mathcal{T} \times H_{\beta^{\prime}} \times D\left(0, \epsilon_{0}\right)$ with $0<\beta^{\prime}<\beta$. Indeed, we can substitute $\mathcal{T}$ by any bounded set in $\mathbb{C}$ in the previous product domain.

The function $f(t, z, \epsilon)$ is constructed as follows. Let $m \mapsto F_{n}(m, \epsilon)$ be a function in $E_{(\beta, \mu)}$ for every $n \geq 0$, depending holomorphically on $\epsilon \in D\left(0, \epsilon_{0}\right)$. We also assume that there exist $C_{F}$ and $T_{0}$ such that (4.6) holds and define $\hat{F}(T, m, \epsilon)=\sum_{n \geq 0} F_{n} T^{n}$.

By construction, for all $\epsilon \in D\left(0, \epsilon_{0}\right), \hat{F}(T, m, \epsilon)$ represents a holomorphic function in $T$ on the disc $D\left(0, T_{0} / 2\right)$ with values in the Banach space $E_{(\beta, \mu)}$. We define

$$
f(t, z, \epsilon)=\mathcal{F}^{-1}(m \mapsto \hat{F}(\epsilon t, m, \epsilon))(z),
$$

which stands for a holomorphic bounded function on $D\left(0, \epsilon_{0} T_{0} / 2\right) \times H_{\beta^{\prime}} \times D\left(0, \epsilon_{0}\right)$ for all $0<\beta^{\prime}<\beta$.

Theorem 5.3 Under the construction made at the beginning of this section of the elements involved in problem (5.1), assume that the above conditions hold. Let $\left(\mathcal{E}_{p}\right)_{0 \leq p \leq \varsigma-1}$ be a good covering in $\mathbb{C}^{\star}$, and let a family $\left\{\left(\mathcal{R}_{\mathfrak{d}_{p}, \tilde{\delta}}\right)_{0 \leq p \leq \varsigma-1}, D(0, \rho), \mathcal{T}\right\}$ be associated with this covering. Then there exist $r_{Q, R_{D_{1}}}, r_{Q, R_{D_{2}}}>0$ large enough and constants $\varsigma_{\Psi}>0$ and $\varsigma_{\varphi}>0$ such that if

$$
\tilde{C}_{\ell} \leq \varsigma_{\varphi} \text { and } C_{\Psi_{1}} \leq \varsigma_{\psi}
$$


for all $1 \leq \ell \leq D-1$, then for every $0 \leq p \leq \varsigma-1$, we can construct a solution $u^{\mathfrak{d}_{p}}(t, z, \epsilon)$ of (5.1) that defines a holomorphic function on $\mathcal{T} \times H_{\beta^{\prime}} \times \mathcal{E}_{p}$ for every $0<\beta^{\prime}<\beta$.

Proof Let $0 \leq p \leq \varsigma-1$ and consider the equation

$$
\begin{aligned}
& Q(i m) \sigma_{q, T} U^{\mathfrak{d}_{p}}(T, m, \epsilon) \\
& =T^{d_{D_{1}}} \sigma_{q, T}^{\frac{d_{D_{1}}}{k_{1}+1}} R_{D_{1}}(i m) U^{\mathfrak{d}_{p}}(T, m, \epsilon)+T^{d_{D_{2}}} \sigma_{q, T}^{\frac{d_{D_{2}}}{k_{2}+1}} R_{D_{2}}(i m) U^{\mathfrak{d}_{p}}(T, m, \epsilon) \\
& \quad+\sum_{\ell=1}^{D-1} \epsilon^{\Delta_{\ell}-d_{\ell}} T^{d_{\ell}} \sigma_{q, T}^{\delta_{\ell}}\left(\frac{1}{(2 \pi)^{1 / 2}} \int_{-\infty}^{+\infty} C_{\ell}\left(T, m-m_{1}, \epsilon\right) R_{\ell}\left(i m_{1}\right) U^{\mathfrak{d}_{p}}\left(T, m_{1}, \epsilon\right) d m_{1}\right) \\
& \quad+\sigma_{q, T} \hat{F}(T, m, \epsilon) .
\end{aligned}
$$

Under an appropriate choice of the constants $\varsigma_{\psi}$ and $\varsigma_{\varphi}$, we can follow the construction in Sect. 4.1 and apply Proposition 4.2 to obtain a solution $U^{\mathfrak{o}_{p}}(T, m, \epsilon)$ of (5.2).

Regarding the properties of the $q$-Laplace transform, from the results obtained in Sect. 4.2 we have that $U^{\mathfrak{d}_{p}}(T, m, \epsilon)$ is the $q$-Laplace transform of order $k_{2}$ of a function $w_{k_{2}}^{\mathfrak{d}_{p}}$ along direction $\mathfrak{d}_{p}$, which depends on $T$. Indeed,

$$
U^{\mathfrak{d}_{p}}(T, m, \epsilon)=\frac{1}{\pi_{q^{1 / k_{2}}}} \int_{L_{\mathfrak{o}_{p}}} \frac{w_{k_{2}}^{\mathfrak{d}_{p}}(u, m, \epsilon)}{\Theta_{q^{1 / k_{2}}}\left(\frac{u}{T}\right)} \frac{d u}{u}
$$

for some $L_{\mathfrak{o}_{p}} \subseteq S_{\mathfrak{o}_{p}} \cup\{0\}$, and $w_{k_{2}}^{\mathfrak{d}_{p}}(\tau, m, \epsilon)$ is a continuous function on $S_{\mathfrak{d}_{p}} \times \mathbb{R} \times D\left(0, \epsilon_{0}\right)$, holomorphic with respect to $(\tau, \epsilon)$ in $S_{\mathfrak{o}_{p}} \times D\left(0, \epsilon_{0}\right)$. In addition, there exists $C_{w_{k_{2}}} \mathrm{o}_{p}>0$ such that

$$
\left|w_{k_{2}}^{\mathfrak{o}_{p}}(\tau, m, \epsilon)\right| \leq C_{w_{k_{2}} \mathfrak{o}_{p}} \frac{1}{(1+|m|)^{\mu}} e^{-\beta|m|} \exp \left(\frac{k_{2}}{2 \log (q)} \log ^{2}|\tau|+\nu \log |\tau|\right)
$$

for some $v \in \mathbb{R}$. This holds for $\tau \in S_{\mathfrak{d}_{p}}, m \in \mathbb{R}$, and $\epsilon \in D\left(0, \epsilon_{0}\right)$. Moreover, in view of Proposition 4.4, the function $w_{k_{2}}^{\mathfrak{d}_{p}}(\tau, m, \epsilon)$ and the $q$-Laplace transform of order $\kappa$ of the function $w_{k_{1}}^{\mathfrak{d}_{p}}(\tau, m, \epsilon)$ along direction $\mathfrak{d}_{p}^{1}$, where $e^{i \mathfrak{d}_{p}^{1}} \mathbb{R}_{+} \subseteq S_{\mathfrak{d}_{p}} \cup\{0\}$, depending on $\tau$, coincide in $\left(S_{\mathfrak{o}_{p}} \cap D\left(0, r_{1}\right)\right) \times \mathbb{R} \times D\left(0, \epsilon_{0}\right)$ for $0<r_{1} \leq q^{\left(\frac{1}{2}-\alpha\right) / \kappa} / 2$ with some $\alpha \in \mathbb{R}$. The function $w_{k_{1}}^{\mathfrak{d}_{p}}(\tau, m, \epsilon)$ is such that for some $C_{w_{k_{1}}} \mathfrak{o}_{p}, \delta>0$,

$$
\left|w_{k_{1}}^{\mathfrak{o}_{p}}(\tau, m, \epsilon)\right| \leq C_{w_{k_{1}}^{\mathfrak{o}_{p} p}} \frac{1}{(1+|m|)^{\mu}} e^{-\beta|m|} \exp \left(\frac{\kappa}{2 \log (q)} \log ^{2}|\tau+\delta|+\alpha \log |\tau+\delta|\right)
$$

for $\tau \in\left(D(0, \rho) \cup U_{\mathfrak{o}_{p}}\right), m \in \mathbb{R}$, and $\epsilon \in D\left(0, \epsilon_{0}\right)$. This function is an extension of a function $w_{k_{1}}(\tau, m, \epsilon)$, common for every $0 \leq p \leq \varsigma-1$, continuous on $\bar{D}(0, \rho) \times \mathbb{R} \times D\left(0, \epsilon_{0}\right)$, and holomorphic with respect to $(\tau, \epsilon)$ in $D(0, \rho) \times D\left(0, \epsilon_{0}\right)$.

The bounds in (5.4) with respect to variable $m$ are transmitted to $U^{\mathfrak{d}_{p}}(T, m, \epsilon)$ as defined in (5.3). This allows us to define the function

$$
\begin{aligned}
u^{\mathfrak{d}_{p}}(t, z, \epsilon) & :=\mathcal{F}^{-1}\left(m \mapsto U^{\mathfrak{d}_{p}}(\epsilon t, m, \epsilon)\right)(z) \\
& =\frac{1}{(2 \pi)^{1 / 2}} \frac{1}{\pi_{q}^{1 / k_{2}}} \int_{-\infty}^{\infty} \int_{L_{\mathfrak{d}_{p}}} \frac{w_{k_{2}}^{\mathfrak{d}_{p}}(u, m, \epsilon)}{\Theta_{q^{1 / k_{2}}}\left(\frac{u}{\epsilon t}\right)} \frac{d u}{u} \exp (i z m) d m,
\end{aligned}
$$


which turns out to be holomorphic on $\mathcal{T} \times H_{\beta^{\prime}} \times \mathcal{E}_{p}$. The properties of the inverse Fourier transform allow us to conclude that $u^{\mathrm{o}_{p}}(t, z, \epsilon)$ is a solution of equation (5.1) defined on $\mathcal{T} \times H_{\beta^{\prime}} \times \mathcal{E}_{p}$.

Proposition 5.4 Let $0 \leq p \leq \varsigma-1$. Under the hypotheses of Theorem 5.3, assume that the unbounded sectors $U_{\mathfrak{d}_{p}}$ and $U_{\mathfrak{d}_{p+1}}$ are wide enough so that $U_{\mathfrak{d}_{p}} \cap U_{\mathfrak{o}_{p+1}}$ contains the sector $U_{\mathfrak{o}_{p}, \mathfrak{d}_{p+1}}=\left\{\tau \in \mathbb{C}^{\star}: \arg (\tau) \in\left[\mathfrak{d}_{p}, \mathfrak{d}_{p+1}\right]\right\}$. Then there exist $K_{1}>0$ and $K_{2} \in \mathbb{R}$ such that

$$
\left|u^{\mathfrak{d}_{p+1}}(t, z, \epsilon)-u^{\mathfrak{d}_{p}}(t, z, \epsilon)\right| \leq K_{1} \exp \left(-\frac{k_{2}}{2 \log (q)} \log ^{2}|\epsilon|\right)|\epsilon|^{K_{2}}
$$

for all $t \in \mathcal{T}, z \in H_{\beta^{\prime}}$, and $\epsilon \in \mathcal{E}_{p} \cap \mathcal{E}_{p+1}$.

Proof Let $0 \leq p \leq \varsigma-1$. Taking into account that $U_{\mathfrak{o}_{p}, \mathfrak{o}_{p+1}} \subseteq U_{\mathfrak{o}_{p}} \cap U_{\mathfrak{o}_{p+1}}$, we observe from the construction of the functions $U^{\mathfrak{o}_{p}}$ and $U^{\mathfrak{o}_{p+1}}$ that $\mathcal{L}_{q ; 1 / \kappa}^{\mathfrak{o}_{p}}\left(w_{k_{1}}^{\mathfrak{o}_{p}}\right)(\tau, m, \epsilon)$ and $\mathcal{L}_{q ; 1 / \kappa}^{\mathfrak{d}_{p+1}}\left(w_{k_{1}}^{\mathfrak{d}_{p+1}}\right)(\tau, m, \epsilon)$ coincide in the domain $\left(\mathcal{R}_{\mathfrak{d}_{p}}^{b} \cap \mathcal{R}_{\mathfrak{d}_{p+1}}^{b}\right) \times \mathbb{R} \times D\left(0, \epsilon_{0}\right)$. This entails the existence of $w_{k_{2}}^{\mathfrak{d}_{p}, \mathfrak{o}_{p+1}}(\tau, m, \epsilon)$ that is holomorphic with respect to $\tau$ on $\mathcal{R}_{\mathfrak{o}_{p}}^{b} \cup \mathcal{R}_{\mathfrak{d}_{p+1}}^{b}$, continuous with respect to $m \in \mathbb{R}$, and holomorphic with respect to $\epsilon$ in $D\left(0, \epsilon_{0}\right)$ and coincides with $\mathcal{L}_{q ; 1 / \kappa}^{\mathfrak{d}_{p}}\left(w_{k_{1}}^{\mathfrak{d}_{p}}\right)(\tau, m, \epsilon)$ on $\mathcal{R}_{\mathfrak{o}_{p}}^{b} \times \mathbb{R} \times D\left(0, \epsilon_{0}\right)$ and also with $\mathcal{L}_{q ; 1 / \kappa}^{\mathfrak{d}_{p+1}}\left(w_{k_{1}}^{\mathfrak{d}_{p+1}}\right)(\tau, m, \epsilon)$ on $\mathcal{R}_{\mathfrak{d}_{p+1}}^{b} \times \mathbb{R} \times D\left(0, \epsilon_{0}\right)$.

Let $\widetilde{\rho}>0$ be such that $\widetilde{\rho} e^{i \mathfrak{d}_{p}} \in \mathcal{R}_{\mathfrak{d}_{p}}^{b}$ and $\widetilde{\rho} e^{i \mathfrak{d}_{p+1}} \in \mathcal{R}_{\mathfrak{d}_{p+1}}^{b}$. The function

$$
u \mapsto \frac{w_{k_{2}}^{\mathfrak{o}_{p}, \mathfrak{d}_{p+1}}(u, m, \epsilon)}{\Theta_{q^{1 / k_{2}}}\left(\frac{u}{\epsilon t}\right)}
$$

is holomorphic on $\mathcal{R}_{\mathfrak{d}_{p}}^{b} \cup \mathcal{R}_{\mathfrak{d}_{p+1}}^{b}$ for all $(m, \epsilon) \in \mathbb{R} \times\left(\mathcal{E}_{p} \cap \mathcal{E}_{p+1}\right)$, and its integral along the closed path constructed by concatenation of the segment starting at the origin and with ending point fixed at $\widetilde{\rho} e^{i \mathrm{~d}_{p}}$, the arc of circle with radius $\widetilde{\rho}$ connecting $\widetilde{\rho} e^{i \mathrm{i}_{p}}$ with $\widetilde{\rho} e^{i \mathfrak{d}_{p+1}} \subseteq \mathcal{R}_{\mathfrak{d}_{p+1}}^{b}$, and the segment from $\tilde{\rho} e^{i \mathfrak{d}_{p+1}}$ to 0 vanishes. The difference $u^{\mathfrak{d}_{p+1}}-u^{\mathfrak{d}_{p}}$ can be written in the form

$$
\begin{aligned}
u^{\mathfrak{d}_{p+1}}(t, z, \epsilon)-u^{\mathfrak{o}_{p}}(t, z, \epsilon) & =\frac{1}{(2 \pi)^{1 / 2}} \frac{1}{\pi_{q^{1 / k_{2}}}} \int_{-\infty}^{\infty} \int_{L_{\mathfrak{d}_{p+1}, \widetilde{\rho}}} \frac{w_{k_{2}}^{\mathfrak{o}_{p+1}}(u, m, \epsilon)}{\Theta_{q^{1 / k_{2}}}\left(\frac{u}{\epsilon t}\right)} \exp (i z m) \frac{d u}{u} d m \\
& -\frac{1}{(2 \pi)^{1 / 2}} \frac{1}{\pi_{q^{1 / k_{2}}}} \int_{-\infty}^{\infty} \int_{L_{\mathfrak{d}_{p}, \widetilde{\rho}}} \frac{w_{k_{2}}^{\mathfrak{o}_{p}}(u, m, \epsilon)}{\Theta_{q^{1 / k_{2}}}\left(\frac{u}{\epsilon t}\right)} \exp (i z m) \frac{d u}{u} d m \\
& +\frac{1}{(2 \pi)^{1 / 2}} \frac{1}{\pi_{q^{1 / k_{2}}}} \int_{-\infty}^{\infty} \int_{\mathcal{C}_{\widetilde{\rho}, \mathfrak{d}_{p}, \mathfrak{d}_{p+1}}} \frac{w_{k_{2}}^{\mathfrak{p}_{p}, \mathfrak{o}_{p+1}}(u, m, \epsilon)}{\Theta_{q^{1 / k_{2}}}\left(\frac{u}{\epsilon t}\right)} \exp (i z m) \frac{d u}{u} d m,
\end{aligned}
$$

where $L_{\mathfrak{o}_{j}, \tilde{\rho}}=[\widetilde{\rho},+\infty) e^{i \mathfrak{d}_{j}}$ for $j \in\{p, p+1\}$, and $\mathcal{C}_{\widetilde{\rho}, \mathfrak{o}_{p}, \mathfrak{o}_{p+1}}$ is the arc of circle connecting $\widetilde{\rho} e^{i \mathfrak{o}_{p}}$ with $\widetilde{\rho} e^{i \mathfrak{D}_{p+1}}$ (see Fig. 2).

Let

$$
I_{1}:=\left|\frac{1}{(2 \pi)^{1 / 2}} \frac{1}{\pi_{q^{1 / k_{2}}}} \int_{-\infty}^{\infty} \int_{L_{\mathfrak{d}_{p+1}, \tilde{\rho}}} \frac{w_{k_{2}}^{\mathfrak{o}_{p+1}}(u, m, \epsilon)}{\Theta_{q^{1 / k_{2}}}\left(\frac{u}{\epsilon t}\right)} \exp (i z m) \frac{d u}{u} d m\right| .
$$


Figure 2 Deformation of the path of integration, first case

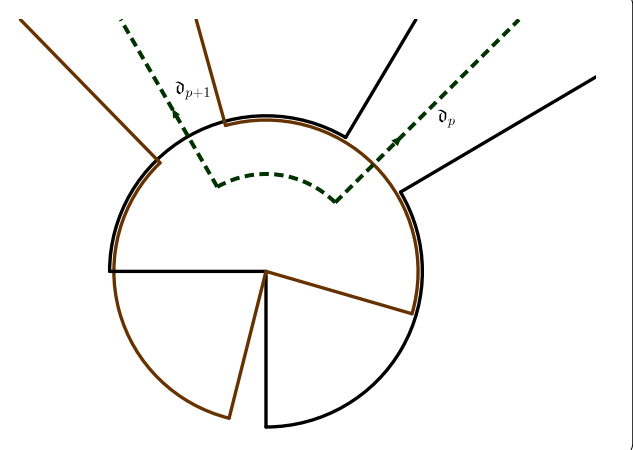

In view of (5.4) and (3.2), we have

$$
\begin{aligned}
I_{1} \leq & \frac{C_{w_{k_{2}}{ }^{{ }^{d} p+1}}}{C_{q, k_{2}} \tilde{\delta}(2 \pi)^{1 / 2}} \frac{|\epsilon t|^{1 / 2}}{\pi_{q^{1 / k_{2}}}} \int_{-\infty}^{\infty} e^{-\beta|m|-m \Im(z)} \frac{d m}{(1+|m|)^{\mu}} \\
& \times \int_{\tilde{\rho}}^{\infty} \exp \left(\frac{k_{2} \log ^{2}|u|}{2 \log (q)}+v \log |u|\right)|u|^{-3 / 2} \exp \left(-\frac{k_{2} \log ^{2}\left(\frac{|u|}{|\epsilon t|}\right)}{2 \log (q)}\right) d|u| .
\end{aligned}
$$

We recall that we have restricted the domain of the variable $z$ so that $|\Im(z)| \leq \beta^{\prime}<\beta$. Then the first integral in the previous expression is convergent, and we derive

$$
I_{1} \leq \frac{\tilde{C}_{w_{k}{ }^{\mathfrak{d} p+1}}}{(2 \pi)^{1 / 2}} \frac{\left(\epsilon_{0} r_{\mathcal{T}}\right)^{1 / 2}}{\pi_{q^{1 / k_{2}}}} \int_{\widetilde{\rho}}^{\infty} \exp \left(\frac{k_{2} \log ^{2}|u|}{2 \log (q)}\right) \exp \left(-\frac{k_{2} \log ^{2}\left(\frac{|u|}{|\epsilon t|}\right)}{2 \log (q)}\right)|u|^{\nu-3 / 2} d|u|
$$

for some $\tilde{C}_{w_{k_{2}} \mathfrak{o}_{p+1}}>0$. We derive

$$
\begin{aligned}
\exp \left(\frac{k_{2} \log ^{2}|u|}{2 \log (q)}\right) \exp \left(-\frac{k_{2} \log ^{2}\left(\frac{|u|}{|\epsilon t|}\right)}{2 \log (q)}\right) \\
=\exp \left(\frac{k_{2}}{2 \log (q)}\left(-\log ^{2}|\epsilon|-2 \log |\epsilon| \log |t|-\log ^{2}|t|\right)\right) \\
\quad \times \exp \left(\frac{k_{2}}{\log (q)}(\log |u| \log |\epsilon|+\log |u| \log |t|)\right) .
\end{aligned}
$$

Since by assumption $0<\epsilon_{0}<1$ and $0<r_{\mathcal{T}}<1$, we get

$$
\begin{aligned}
& \exp \left(-\frac{k_{2}}{\log (q)} \log |\epsilon| \log |t|\right) \leq|\epsilon|^{-\frac{k_{2}}{\log (q)} \log \left(r_{\mathcal{T}}\right)}, \\
& \exp \left(\frac{k_{2}}{\log (q)} \log |u| \log |\epsilon|\right) \leq|\epsilon|^{\frac{k_{2}}{\log (q)} \log (\widetilde{\rho})}
\end{aligned}
$$

for $t \in \mathcal{T}, \epsilon \in \mathcal{E}_{p} \cap \mathcal{E}_{p+1}$, and $|u| \geq \widetilde{\rho}$, and also, for $t \in \mathcal{T}$,

$$
\begin{aligned}
& \exp \left(\frac{k_{2}}{\log (q)} \log |u| \log |t|\right) \leq|t|^{\frac{k_{2}}{\log (q)} \log (\widetilde{\rho})} \text { if } \widetilde{\rho} \leq|u| \leq 1 \\
& \exp \left(\frac{k_{2}}{\log (q)} \log |u| \log |t|\right) \leq|u|^{\frac{k_{2}}{\log (q)} \log \left(r_{\mathcal{T}}\right)} \text { if }|u| \geq 1
\end{aligned}
$$


In addition, there exists $K_{k_{2}, \tilde{\rho}, q}>0$ such that

$$
\sup _{x>0} x^{\frac{k_{2}}{\log (q)} \log (\widetilde{\rho})} \exp \left(-\frac{k_{2}}{2 \log (q)} \log ^{2}(x)\right) \leq K_{k_{2}, \widetilde{\rho}, q}
$$

In view of (5.8), (5.9), and (5.10), bearing in mind the inequalities of Definition 5.2, we deduce that there exist $\tilde{K}^{1} \in \mathbb{R}$ and $\tilde{K}^{2}>0$ such that

$$
\exp \left(\frac{k_{2} \log ^{2}|u|}{2 \log (q)}\right) \exp \left(-\frac{k_{2} \log ^{2}\left(\frac{|u|}{|\epsilon t|}\right)}{2 \log (q)}\right)|u|^{\nu} \leq \tilde{K}^{2} \exp \left(-\frac{k_{2}}{2 \log (q)} \log ^{2}|\epsilon|\right)|\epsilon|^{\tilde{K}^{1}}
$$

for $t \in \mathcal{T}, r \geq \widetilde{\rho}$, and $\epsilon \in \mathcal{E}_{p} \cap \mathcal{E}_{p+1}$. From the last inequality we arrive at

$$
\begin{aligned}
I_{1} & \leq \frac{\tilde{K}^{2} \tilde{C}_{w_{2}}^{\mathrm{o}_{p+1}}}{(2 \pi)^{1 / 2}} \frac{\left(\epsilon_{0} r_{\tau}\right)^{1 / 2}}{\pi_{q^{1 / k_{2}}}} \int_{\widetilde{\rho}}^{\infty} \frac{d|u|}{|u|^{3 / 2}} \exp \left(-\frac{k_{2}}{2 \log (q)} \log ^{2}|\epsilon|\right)|\epsilon|^{\tilde{K}^{1}} \\
& =\tilde{K}^{3} \exp \left(-\frac{k_{2}}{2 \log (q)} \log ^{2}|\epsilon|\right)|\epsilon|^{\tilde{K}^{1}}
\end{aligned}
$$

for some $\tilde{K}^{3}>0$ and for all $t \in \mathcal{T}, z \in H_{\beta^{\prime}}$, and $\epsilon \in \mathcal{E}_{p} \cap \mathcal{E}_{p+1}$.

We can estimate in the same manner the expression

$$
I_{2}:=\left|\frac{1}{(2 \pi)^{1 / 2}} \frac{1}{\pi_{q^{1 / k_{2}}}} \int_{-\infty}^{\infty} \int_{L_{\mathfrak{o}_{p}, \tilde{\rho}}} \frac{w_{k_{2}}^{\mathfrak{o}_{p}}(u, m, \epsilon)}{\Theta_{q^{1 / k_{2}}}\left(\frac{u}{\epsilon t}\right)} \exp (i z m) \frac{d u}{u} d m\right|
$$

to arrive at the existence of $\tilde{K}^{4}>0$ such that

$$
I_{2} \leq \tilde{K}^{4} \exp \left(-\frac{k_{2}}{2 \log (q)} \log ^{2}|\epsilon|\right)|\epsilon|^{\tilde{K}^{1}}
$$

for all $t \in \mathcal{T}, z \in H_{\beta^{\prime}}$, and $\epsilon \in \mathcal{E}_{p} \cap \mathcal{E}_{p+1}$. We now provide upper bounds for the quantity

$$
I_{3}:=\left|\frac{1}{(2 \pi)^{1 / 2}} \frac{1}{\pi_{q^{1 / k_{2}}}} \int_{-\infty}^{\infty} \int_{\mathcal{C}_{\widetilde{\rho}, \mathfrak{d}_{p}, \mathfrak{o}_{p+1}}} \frac{w_{k_{2}}^{\mathfrak{d}_{p}, \mathfrak{d}_{p+1}}(u, m, \epsilon)}{\Theta_{q^{1 / k_{2}}}\left(\frac{u}{\epsilon t}\right)} \exp (i z m) \frac{d u}{u} d m\right| .
$$

From the construction of $w_{k_{2}}^{\mathfrak{o}_{p}, \mathrm{~d}_{p+1}}(\tau, m, \epsilon)$ we have

$$
\left|w_{k_{2}}^{\mathfrak{d}_{p} \mathfrak{d}_{p+1}}(u, m, \epsilon)\right| \leq \tilde{C}_{w_{k_{1}}^{\mathfrak{o}_{p}}} \frac{1}{(1+|m|)^{\mu}} e^{-\beta|m|}
$$

for some $\tilde{C}_{w_{k_{1}}^{\mathfrak{o}_{p}}}>0$ and for all $u \in \mathcal{C}_{\widetilde{\rho}, \mathfrak{d}_{p}, \mathfrak{d}_{p+1}}, m \in \mathbb{R}$, and $\epsilon \in D\left(0, \epsilon_{0}\right)$.

The estimates with (3.2) allow us to obtain the existence of $\tilde{C}_{w_{k_{2}}}^{\mathrm{o}_{p}, \mathfrak{d}_{p+1}}>0$ such that

$$
I_{3} \leq \tilde{C}_{w_{k_{2}}}^{\mathfrak{d}_{p}, \mathfrak{d}_{p+1}} \int_{-\infty}^{\infty} \frac{e^{-\beta|m|-m \Im(z)}}{(1+|m|)^{\mu}} d m\left|\mathfrak{d}_{p+1}-\mathfrak{d}_{p}\right||t|^{1 / 2} \exp \left(-\frac{k_{2} \log ^{2}\left(\frac{\tilde{\rho}}{|\epsilon t|}\right)}{2 \log (q)}\right)
$$


for all $t \in \mathcal{T}, z \in H_{\beta^{\prime}}$, and $\epsilon \in \mathcal{E}_{p} \cap \mathcal{E}_{p+1}$. We can follow analogous arguments as in the previous steps to provide upper estimates of the expression

$$
|t|^{1 / 2} \exp \left(-\frac{k_{2} \log ^{2}\left(\frac{\widetilde{\rho}}{|\epsilon t|}\right)}{2 \log (q)}\right)
$$

Indeed,

$$
\begin{aligned}
& |t|^{1 / 2} \exp \left(-\frac{k_{2} \log ^{2}\left(\frac{\widetilde{\rho}}{|\epsilon t|}\right)}{2 \log (q)}\right) \\
& =\exp \left(-\frac{k_{2} \log ^{2}(\widetilde{\rho})}{2 \log (q)}\right)|\epsilon|^{\frac{k_{2} \log (\widetilde{\rho})}{\log (q)}}|t|^{\frac{k_{2} \log (\widetilde{\rho})}{\log (q)}} \\
& \quad \times \exp \left(\frac{k_{2}}{2 \log (q)}\left(-\log ^{2}|\epsilon|-2 \log |\epsilon| \log |t|-\log ^{2}|t|\right)\right)|t|^{1 / 2}
\end{aligned}
$$

Since by assumption $0 \leq \epsilon_{0}<1$, we check that

$$
\exp \left(-\frac{k_{2}}{\log (q)} \log |\epsilon| \log |t|\right) \leq|\epsilon|^{-\frac{k_{2}}{\log (q)} \log \left(r_{\mathcal{T}}\right)}
$$

for $t \in \mathcal{T}$ and $\epsilon \in \mathcal{E}_{p} \cap \mathcal{E}_{p+1}$. Gathering (5.10), we get the existence of $\tilde{K}^{5} \in \mathbb{R}$ and $\tilde{K}^{6}>0$ such that

$$
|t|^{1 / 2} \exp \left(-\frac{k_{2} \log ^{2}\left(\frac{\tilde{\rho}}{|\epsilon t|}\right)}{2 \log (q)}\right) \leq \tilde{K}^{6} \exp \left(-\frac{k_{2}}{2 \log (q)} \log ^{2}|\epsilon|\right)|\epsilon|^{\tilde{K}^{5}}
$$

to conclude that

$$
I_{3} \leq \tilde{K}^{7} \exp \left(-\frac{k_{2}}{2 \log (q)} \log ^{2}|\epsilon|\right)|\epsilon|^{\tilde{K}^{5}}
$$

for some $\tilde{K}^{7}>0$ and all $t \in \mathcal{T}, z \in H_{\beta^{\prime}}$, and $\epsilon \in \mathcal{E}_{p} \cap \mathcal{E}_{p+1}$. We conclude the proof of this result in view of (5.11), (5.12), (5.13), and decomposition (5.7).

Lemma 5.5 Let $0 \leq p \leq \varsigma-1$. Under the hypotheses of Theorem 5.3, assume that $U_{\mathfrak{o}_{p}} \cap U_{\mathfrak{o}_{p+1}}=\emptyset$. Then there exist $K_{p}^{\mathcal{L}}>0$ and $M_{p}^{\mathcal{L}} \in \mathbb{R}$ such that

$$
\begin{aligned}
& \left|\mathcal{L}_{q ; 1 / \kappa}^{\mathfrak{o}_{p+1}}\left(w_{k_{1}}^{\mathfrak{o}_{p+1}}\right)(\tau, m, \epsilon)-\mathcal{L}_{q ; 1 / \kappa}^{\mathfrak{o}_{p}}\left(w_{k_{1}}^{\mathrm{o}_{p}}\right)(\tau, m, \epsilon)\right| \\
& \quad \leq K_{p}^{\mathcal{L}} e^{-\beta|m|}(1+|m|)^{-\mu} \exp \left(-\frac{\kappa}{2 \log (q)} \log ^{2}|\tau|\right)|\tau|^{M_{p}^{\mathcal{L}}}
\end{aligned}
$$

for all $\epsilon \in\left(\mathcal{E}_{p} \cap \mathcal{E}_{p+1}\right), \tau \in\left(\mathcal{R}_{\mathfrak{d}_{p}}^{b} \cap \mathcal{R}_{\mathfrak{o}_{p+1}}^{b}\right)$, and $m \in \mathbb{R}$.

Proof We first recall that, without loss of generality, the intersection $\mathcal{R}_{\mathfrak{o}_{p}}^{b} \cap \mathcal{R}_{\mathfrak{o}_{p+1}}^{b}$ can be assumed to be a nonempty set because we can vary $\tilde{\delta}$ in advance to be as close to 0 as desired. 
Analogous arguments as in the beginning of the proof of Proposition 5.4 allow us to write

$$
\begin{aligned}
\mathcal{L}_{q ; 1 / \kappa}^{\mathfrak{d}_{p+1}}\left(w_{k_{1}}^{\mathfrak{d}_{p+1}}\right)(\tau, m, \epsilon)-\mathcal{L}_{q ; 1 / \kappa}^{\mathfrak{o}_{p}}\left(w_{k_{1}}^{\mathfrak{o}_{p}}\right)(\tau, m, \epsilon) \\
=\frac{1}{\pi_{q^{1 / \kappa}}} \int_{L_{\mathfrak{o}_{p+1}, \tilde{\rho}}} \frac{w_{k_{1}}^{\mathfrak{o}_{p+1}}(u, m, \epsilon)}{\Theta_{q^{1 / \kappa}}\left(\frac{u}{\tau}\right)} \frac{d u}{u} \\
\quad-\frac{1}{\pi_{q^{1 / \kappa}}} \int_{L_{\mathfrak{d}_{p}, \tilde{\rho}}} \frac{w_{k_{1}}^{\mathfrak{o}_{p}}(u, m, \epsilon)}{\Theta_{q^{1 / \kappa}}\left(\frac{u}{\tau}\right)} \frac{d u}{u} \\
\quad+\frac{1}{\pi_{q^{1 / \kappa}}} \int_{\mathcal{C}_{\tilde{\rho}, \mathfrak{o}_{p}, \mathfrak{o}_{p+1}}} \frac{w_{k_{1}}(u, m, \epsilon)}{\Theta_{q^{1 / \kappa}}\left(\frac{u}{\tau}\right)} \frac{d u}{u}
\end{aligned}
$$

where $\widetilde{\rho}, L_{\mathfrak{o}_{p}, \widetilde{\rho}}, L_{\mathfrak{d}_{p+1}, \widetilde{\rho}}$, and $\mathcal{C}_{\widetilde{\rho}, \mathfrak{d}_{p}, \mathfrak{o}_{p+1}}$ are constructed in Proposition 5.4.

In view of (5.5) and (3.2), we have

$$
\begin{aligned}
I_{1}^{\mathcal{L}} & :=\left|\frac{1}{\pi_{q^{1 / \kappa}}} \int_{L_{\mathfrak{d}_{p}, \widetilde{\rho}}} \frac{w_{k_{1}}^{\mathfrak{o}_{p}}(u, m, \epsilon)}{\Theta_{q^{1 / \kappa}}\left(\frac{u}{\tau}\right)} \frac{d u}{u}\right| \\
& \leq \frac{C_{w_{k_{1}}^{\mathrm{o}_{p}}}}{C_{q, \kappa} \tilde{\delta}} \frac{|\tau|^{1 / 2}}{(1+|m|)^{\mu}} e^{-\beta|m|} \int_{\widetilde{\rho}}^{\infty} \frac{\exp \left(\frac{\kappa \log ^{2}\left|r e^{i \mathfrak{D}_{p}}+\delta\right|}{2 \log (q)}+\alpha \log \left|r e^{i \mathfrak{D}_{p}}+\delta\right|\right)}{\exp \left(\frac{\kappa}{2} \frac{\log ^{2}\left(\frac{r}{|\tau|}\right)}{\log (q)}\right)} \frac{d r}{r^{3 / 2}} \\
& \leq K_{p, 1}^{\mathcal{L}}|\tau|^{1 / 2}(1+|m|)^{-\mu} e^{-\beta|m|} \int_{\widetilde{\rho}}^{\infty} \frac{\exp \left(\frac{\kappa \log ^{2} r}{2 \log (q)}+\alpha \log r\right)}{\exp \left(\frac{\kappa}{2} \frac{\log ^{2}\left(\frac{r}{|\tau|}\right)}{\log (q)}\right)} \frac{d r}{r^{3 / 2}}
\end{aligned}
$$

for some $K_{p, 1}^{\mathcal{L}}>0$. Taking into account the choice of $\alpha$ in Definition 5.2, by usual calculations we derive that the previous expression equals

$$
K_{p, 1}^{\mathcal{L}}|\tau|^{1 / 2}(1+|m|)^{-\mu} e^{-\beta|m|} \exp \left(-\frac{\kappa}{2 \log (q)} \log ^{2}|\tau|\right) \int_{\widetilde{\rho}}^{\infty} r^{\frac{\kappa \log |\tau|}{\log (q)}+\alpha-3 / 2} d r
$$

Besides, we observe from direct computations that

$$
\int_{\tilde{\rho}}^{\infty} r^{\frac{\kappa \log |\tau|}{\log (q)}+\alpha-\frac{3}{2}} d r
$$

is upper bounded by a constant times $|\tau|^{\Delta}$ for every $\Delta<\frac{\kappa}{\log (q)} \log (\tilde{\rho})$. This yields

$$
I_{1}^{\mathcal{L}} \leq K_{p, 2}^{\mathcal{L}}(1+|m|)^{-\mu} e^{-\beta|m|} \exp \left(-\frac{\kappa}{2 \log (q)} \log ^{2}|\tau|\right)|\tau|^{\Delta+\frac{1}{2}}
$$

for some $K_{p, 2}^{\mathcal{L}}>0$. Analogous arguments allow us to obtain the existence of $K_{p, 3}^{\mathcal{L}}>0$ such that

$$
\begin{aligned}
I_{2}^{\mathcal{L}} & :=\left|\frac{1}{\pi_{q^{1 / \kappa}}} \int_{L_{\mathfrak{d}_{p+1}, \tilde{\rho}}} \frac{w_{k_{1}}^{\mathfrak{o}_{p+1}}(u, m, \epsilon)}{\Theta_{q^{1 / \kappa}}\left(\frac{u}{\tau}\right)} \frac{d u}{u}\right| \\
& \leq K_{p, 3}^{\mathcal{L}}(1+|m|)^{-\mu} e^{-\beta|m|} \exp \left(-\frac{\kappa}{2 \log (q)} \log ^{2}|\tau|\right)|\tau|^{\Delta+\frac{1}{2}}
\end{aligned}
$$

for $\Delta$ as before. 
We write

$$
I_{3}^{\mathcal{L}}:=\left|\frac{1}{\pi_{q^{1 / \kappa}}} \int_{\mathcal{C}_{\tilde{\rho}, \mathfrak{d} p, \mathfrak{d} p+1}} \frac{w_{k_{1}}(u, m, \epsilon)}{\Theta_{q^{1 / \kappa}}\left(\frac{u}{\tau}\right)} \frac{d u}{u}\right| .
$$

Regarding (5.5) and (3.2), we derive that

$$
\begin{aligned}
I_{3}^{\mathcal{L}} & \leq \frac{C_{w_{k_{1}} \mathfrak{d}_{p}}}{\pi_{q^{1 / \kappa}}} \frac{e^{-\beta|m|}}{(1+|m|)^{\mu}} \frac{|\tau|^{1 / 2}}{\widetilde{\rho}^{1 / 2} C_{q, \kappa} \tilde{\delta}} \int_{\mathfrak{d}_{p}}^{\mathfrak{d}_{p+1}} \frac{\exp \left(\frac{\kappa \log ^{2}\left|\widetilde{\rho} e^{i \theta}+\delta\right|}{2 \log (q)}+\alpha \log \left|\widetilde{\rho} e^{i \theta}+\delta\right|\right)}{\exp \left(\frac{\kappa}{2} \frac{\log ^{2}\left(\frac{\widetilde{\rho}}{|\tau|}\right)}{\log (q)}\right)} d \theta \\
& \leq K_{p, 4}^{\mathcal{L}}|\tau|^{1 / 2} \frac{e^{-\beta|m|}}{(1+|m|)^{\mu}} \exp \left(-\frac{\kappa}{2} \frac{\log ^{2}\left(\frac{\widetilde{\rho}}{|\tau|}\right)}{\log (q)}\right)
\end{aligned}
$$

with

$$
K_{p, 4}^{\mathcal{L}}=\left|\mathfrak{d}_{p+1}-\mathfrak{d}_{p}\right| \frac{C_{w_{k_{1}} \mathfrak{o}_{p}}}{\pi_{q^{1 / \kappa}}} \frac{1}{\widetilde{\rho}^{1 / 2} C_{q, \kappa} \tilde{\delta}} \exp \left(\frac{\kappa \log ^{2}(\widetilde{\rho}+\delta)}{2 \log (q)}+\alpha \log (\widetilde{\rho}+\delta)\right)
$$

Let $K_{p, 5}^{\mathcal{L}}=K_{p, 4}^{\mathcal{L}} \exp \left(-\frac{\kappa}{2 \log (q)} \log ^{2}(\widetilde{\rho})\right)$. It is straightforward to check that

$$
I_{3}^{\mathcal{L}} \leq K_{p, 5}^{\mathcal{L}}|\tau|^{1 / 2+\frac{\kappa \log (\widetilde{\rho})}{\log (q)}} \frac{e^{-\beta|m|}}{(1+|m|)^{\mu}} \exp \left(-\frac{\kappa}{2} \frac{\log ^{2}|\tau|}{\log (q)}\right)
$$

Substitution of (5.15), (5.16), and (5.17) into (5.14) yields the result.

Proposition 5.6 Let $0 \leq p \leq \varsigma-1$. Under the hypotheses of Theorem 5.3, assume that $U_{\mathfrak{d}_{p}} \cap U_{\mathfrak{d}_{p+1}}=\emptyset$. Then there exist $K_{3}>0$ and $K_{4} \in \mathbb{R}$ such that

$$
\left|u^{\mathfrak{d} p+1}(t, z, \epsilon)-u^{\mathfrak{d}_{p}}(t, z, \epsilon)\right| \leq K_{3} \exp \left(-\frac{k_{1}}{2 \log (q)} \log ^{2}|\epsilon|\right)|\epsilon|^{K_{4}}
$$

for all $t \in \mathcal{T}, z \in H_{\beta^{\prime}}$, and $\epsilon \in \mathcal{E}_{p} \cap \mathcal{E}_{p+1}$.

Proof Let $0 \leq p \leq \varsigma-1$. Under the assumptions of the statement, we observe that we cannot proceed as in the proof of Proposition 5.4 since there does not exist a common function for both indices $p$ and $p+1$, defined in $\mathcal{R}_{\mathfrak{d}_{p}}^{b} \cup \mathcal{R}_{\mathfrak{d}_{p+1}}^{b}$ in the variable of integration when applying the $q$-Laplace transform. However, we can use the analytic continuation property and write the difference $u^{\mathfrak{d}_{p+1}}-u^{\mathfrak{d}_{p}}$ as follows. Let $\tilde{\rho}>0$ be such that $\tilde{\rho} e^{i \mathfrak{d}_{p}} \in \mathcal{R}_{\mathfrak{d}_{p}}^{b}$ and $\tilde{\rho} e^{i \mathfrak{d}_{p+1}} \in \mathcal{R}_{\mathfrak{d}_{p+1}}^{b}$, and let $\theta_{p, p+1} \in \mathbb{R}$ be such that $\tilde{\rho} e^{\theta_{p, p+1}}$ lies in both $\mathcal{R}_{\mathfrak{d}_{p}}^{b}$ and $\mathcal{R}_{\mathfrak{d}_{p+1}}^{b}$. We write $u^{\mathfrak{d}_{p+1}}(t, z, \epsilon)-u^{\mathfrak{d}_{p}}(t, z, \epsilon)$ as follows:

$$
\begin{aligned}
u^{\mathfrak{d}_{p+1}}(t, z, \epsilon)-u^{\mathfrak{d}_{p}}(t, z, \epsilon) & =\frac{1}{(2 \pi)^{1 / 2}} \frac{1}{\pi_{q^{1 / k_{2}}}} \int_{-\infty}^{\infty} \int_{L_{\mathfrak{d}_{p+1}, \tilde{\rho}}} \frac{w_{k_{2}}^{\mathfrak{d}_{p+1}}(u, m, \epsilon)}{\Theta_{q^{1 / k_{2}}\left(\frac{u}{\epsilon t}\right)} \exp (i z m) \frac{d u}{u} d m} \\
& -\frac{1}{(2 \pi)^{1 / 2}} \frac{1}{\pi_{q^{1 / k_{2}}}} \int_{-\infty}^{\infty} \int_{L_{\mathfrak{d}_{p}, \tilde{\rho}}} \frac{w_{k_{2}}^{\mathfrak{d}_{p}}(u, m, \epsilon)}{\Theta_{q^{1 / k_{2}}}\left(\frac{u}{\epsilon t}\right)} \exp (i z m) \frac{d u}{u} d m
\end{aligned}
$$


Figure 3 Deformation of the path of integration, second case

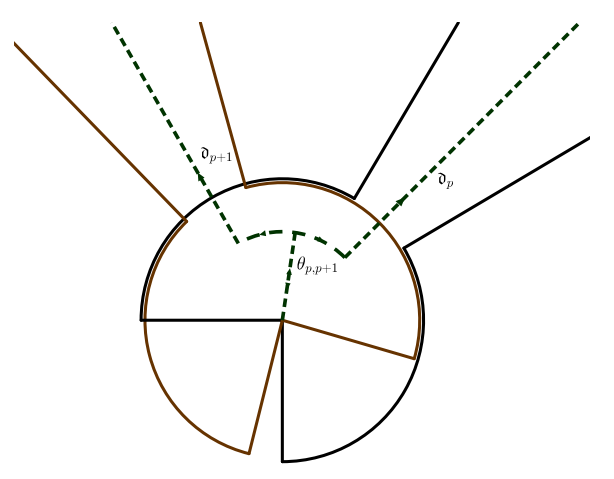

$$
\begin{aligned}
& -\frac{1}{(2 \pi)^{1 / 2}} \frac{1}{\pi_{q^{1 / k_{2}}}} \int_{-\infty}^{\infty} \int_{\mathcal{C}_{\tilde{\rho}, \theta_{p, p+1}, \mathfrak{o}_{p+1}}} \frac{w_{k_{2}}^{\mathfrak{d}_{p}, \mathfrak{o}_{p+1}}(u, m, \epsilon)}{\Theta_{q^{1 / k_{2}}\left(\frac{u}{\epsilon t}\right)} \exp (i z m) \frac{d u}{u} d m} \\
& +\frac{1}{(2 \pi)^{1 / 2}} \frac{1}{\pi_{q^{1 / k_{2}}}} \int_{-\infty}^{\infty} \int_{\mathcal{C}_{\tilde{\rho}, \theta_{p, p+1}, \mathfrak{o}_{p}}} \frac{w_{k_{2}}^{\mathfrak{d}_{p}, \mathfrak{o}_{p+1}}(u, m, \epsilon)}{\Theta_{q^{1 / k_{2}}}\left(\frac{u}{\epsilon t}\right)} \exp (i z m) \frac{d u}{u} d m \\
& +\frac{1}{(2 \pi)^{1 / 2}} \frac{1}{\pi_{q^{1 / k_{2}}}} \int_{-\infty}^{\infty} \int_{L_{0, \tilde{\rho}, \theta_{p, p+1}}} \frac{\mathcal{L}_{q ; 1 / \kappa}^{\mathfrak{d}_{p+1}}\left(w_{k_{1}}^{\mathfrak{d}_{p+1}}\right)\left(\tau, m_{1}, \epsilon\right)-\mathcal{L}_{q ; 1 / \kappa}^{\mathfrak{d}_{p}}\left(w_{k_{1}}^{\mathfrak{d}_{p}}\right)(\tau, m, \epsilon)}{\Theta_{q^{1 / k_{2}}}\left(\frac{u}{\epsilon t}\right)} \\
& \times \exp (i z m) \frac{d u}{u} d m .
\end{aligned}
$$

Here we have denoted $L_{\mathfrak{d}_{j}, \tilde{\rho}}=[\widetilde{\rho},+\infty) e^{i \mathfrak{d}_{j}}$ for $j \in\{p, p+1\}, \mathcal{C}_{\widetilde{\rho}, \theta_{p, p+1}, \mathfrak{o}_{p+1}}$ is the arc of circle connecting $\widetilde{\rho} e^{i \mathfrak{d}_{p+1}}$ with $\tilde{\rho} e^{i \theta_{p, p+1}}, \mathcal{C}_{\widetilde{\rho}, \theta_{p, p+1}, \mathfrak{o}_{p}}$ is the arc of circle connecting $\tilde{\rho} e^{i \mathrm{~d}_{p}}$ with $\tilde{\rho} e^{i \theta_{p, p+1}}$, and $L_{0, \tilde{\rho}, \theta_{p, p+1}}=[0, \widetilde{\rho}] e^{i \theta_{p, p+1}}$, as it is shown in Fig. 3 .

Following the same line of arguments as those in the proof of Proposition 5.4, we can guarantee the existence of $\hat{K}^{j}>0$ and $\hat{K}^{k} \in \mathbb{R}$ for $1 \leq j \leq 4$ and $5 \leq k \leq 8$ such that

$$
\begin{aligned}
& J_{1}:=\left|\frac{1}{(2 \pi)^{1 / 2}} \frac{1}{\pi_{q^{1 / k_{2}}}} \int_{-\infty}^{\infty} \int_{L_{\mathfrak{d}_{p+1}, \tilde{\rho}}} \frac{w_{k_{2}}^{\mathfrak{d}_{p+1}}(u, m, \epsilon)}{\Theta_{q^{1 / k_{2}}}\left(\frac{u}{\epsilon t}\right)} \exp (i z m) \frac{d u}{u} d m\right| \\
& \leq \hat{K}_{1} \exp \left(-\frac{k_{2}}{2 \log (q)} \log ^{2}|\epsilon|\right)|\epsilon|^{\hat{K}^{5}} \\
& J_{2}:=\left|\frac{1}{(2 \pi)^{1 / 2}} \frac{1}{\pi_{q^{1 / k_{2}}}} \int_{-\infty}^{\infty} \int_{L_{\mathfrak{d} p, \tilde{\rho}}} \frac{w_{k_{2}}^{\mathfrak{o}_{p}}(u, m, \epsilon)}{\Theta_{q^{1 / k_{2}}}\left(\frac{u}{\epsilon t}\right)} \exp (i z m) \frac{d u}{u} d m\right| \\
& \leq \hat{K}_{2} \exp \left(-\frac{k_{2}}{2 \log (q)} \log ^{2}|\epsilon|\right)|\epsilon|^{\hat{K}^{6}} \\
& J_{3}:=\left|\frac{1}{(2 \pi)^{1 / 2}} \frac{1}{\pi_{q^{1 / k_{2}}}} \int_{-\infty}^{\infty} \int_{\mathcal{C}_{\widetilde{\rho}, \theta_{p, p+1}, \mathfrak{o}_{p+1}}} \frac{w_{k_{2}}^{\mathfrak{d}_{p}, \mathfrak{o}_{p+1}}(u, m, \epsilon)}{\Theta_{q^{1 / k_{2}}}\left(\frac{u}{\epsilon t}\right)} \exp (i z m) \frac{d u}{u} d m\right| \\
& \leq \hat{K}_{3} \exp \left(-\frac{k_{2}}{2 \log (q)} \log ^{2}|\epsilon|\right)|\epsilon|^{\hat{K}^{7}} \\
& J_{4}:=\left|\frac{1}{(2 \pi)^{1 / 2}} \frac{1}{\pi_{q^{1 / k_{2}}}} \int_{-\infty}^{\infty} \int_{\mathcal{C}_{\widetilde{\rho}, \theta_{p, p+1}, \mathfrak{o}_{p}}} \frac{w_{k_{2}}^{\mathfrak{d}_{p}, \mathfrak{d}_{p+1}}(u, m, \epsilon)}{\Theta_{q^{1 / k_{2}}}\left(\frac{u}{\epsilon t}\right)} \exp (i z m) \frac{d u}{u} d m\right| \\
& \leq \hat{K}_{4} \exp \left(-\frac{k_{2}}{2 \log (q)} \log ^{2}|\epsilon|\right)|\epsilon|^{\hat{K}^{8}} \text {. }
\end{aligned}
$$


We now give estimates for

$$
\begin{aligned}
J_{5}:= & \frac{1}{(2 \pi)^{1 / 2}} \frac{1}{\pi_{q^{1 / k_{2}}}} \\
& \times\left|\int_{-\infty}^{\infty} \int_{L_{0, \tilde{\rho}, \theta_{p, p+1}}} \frac{\mathcal{L}_{q ; 1 / \kappa}^{\mathfrak{d}_{p+1}}\left(w_{k_{1}}^{\mathfrak{d}_{p+1}}\right)(u, m, \epsilon)-\mathcal{L}_{q ; 1 / \kappa}^{\mathfrak{d}_{p}}\left(w_{k_{1}}^{\mathfrak{d}_{p}}\right)(u, m, \epsilon)}{\Theta_{q^{1 / k_{2}}}\left(\frac{u}{\epsilon t}\right)} \exp (i z m) \frac{d u}{u} d m\right| .
\end{aligned}
$$

In view of Lemma 5.5 and (3.2), we have

$$
J_{5} \leq \frac{K_{p}^{\mathcal{L}}}{(2 \pi)^{1 / 2}} \frac{1}{\pi_{q^{1 / k_{2}}}} \int_{-\infty}^{\infty} e^{-\beta|m|-\Im(z) m} \frac{d m}{(1+|m|)^{\mu}} \int_{0}^{\widetilde{\rho}} \frac{\exp \left(-\frac{\kappa}{2 \log (q)} \log ^{2}|u|\right)|u|^{M_{p}^{\mathcal{L}}}}{C_{q, k_{2}} \tilde{\delta} \exp \left(\frac{k_{2}}{2} \frac{\log ^{2}\left|\frac{u}{\epsilon t}\right|}{\log (q)}\right)\left|\frac{u}{\epsilon t}\right|^{1 / 2}} \frac{d|u|}{|u|}
$$

We recall that $z \in H_{\beta^{\prime}}$ for some $\beta^{\prime}<\beta$. Then there exists $K_{31}>0$ such that

$$
J_{5} \leq \frac{K_{p}^{\mathcal{L}} K_{31}}{(2 \pi)^{1 / 2}} \frac{|\epsilon|^{1 / 2} r_{\mathcal{T}}^{1 / 2}}{\pi_{q^{1 / k_{2}}} C_{q, k_{2}} \tilde{\delta}} \int_{0}^{\tilde{\rho}} \frac{\exp \left(-\frac{\kappa}{2 \log (q)} \log ^{2}|u|\right)|u|^{M_{\tilde{p}}^{\mathcal{L}}}}{\exp \left(\frac{k_{2}}{2} \frac{\log ^{2}\left|\frac{u}{\epsilon \epsilon}\right|}{\log (q)}\right)} \frac{d|u|}{|u|^{3 / 2}}
$$

We now proceed to prove that the expression

$$
\int_{0}^{\tilde{\rho}} \frac{\exp \left(-\frac{\kappa}{2 \log (q)} \log ^{2}|u|\right)}{\exp \left(\frac{k_{2}}{2} \frac{\left.\log ^{2} \mid \frac{u}{\epsilon t \mid}\right)}{\log (q)}\right)} \exp \left(\frac{k_{1}}{2 \log (q)} \log ^{2}|\epsilon|\right) \frac{d|u|}{|u|^{3 / 2-M_{\tilde{p}}^{\mathcal{L}}}}
$$

is upper bounded by a positive constant times a certain power of $|\epsilon|$ for all $\epsilon \in\left(\mathcal{E}_{p} \cap \mathcal{E}_{p+1}\right)$ and $t \in \mathcal{T}$. This implies the existence of $K_{32}>0$ such that

$$
J_{5} \leq K_{32}|\epsilon|^{1 / 2} \exp \left(-\frac{k_{1}}{2 \log (q)} \log ^{2}|\epsilon|\right)
$$

for all $\epsilon \in\left(\mathcal{E}_{p} \cap \mathcal{E}_{p+1}\right), t \in \mathcal{T}$, and $z \in H_{\beta^{\prime}}$.

Indeed, we have that

$$
\int_{0}^{\tilde{\rho}} \frac{\exp \left(-\frac{\kappa}{2 \log (q)} \log ^{2}|u|\right)}{\exp \left(\frac{k_{2}}{2} \frac{\log ^{2}\left(\frac{|u|}{|\epsilon t|}\right)}{\log (q)}\right)} \exp \left(\frac{k_{1}}{2 \log (q)} \log ^{2}|\epsilon|\right) \frac{d|u|}{|u|^{3 / 2-M_{p}^{\mathcal{L}}}}
$$

equals

$$
\begin{aligned}
& \exp \left(\frac{k_{1}}{2 \log (q)} \log ^{2}|\epsilon|-\frac{k_{2}}{2 \log (q)} \log ^{2}|\epsilon t|\right) \\
& \quad \times \int_{0}^{\widetilde{\rho}} \exp \left(-\frac{\left(\kappa+k_{2}\right)}{2 \log (q)} \log ^{2}|u|\right)|u|^{\frac{k_{2} \log |\epsilon t|}{\log (q)}-\frac{3}{2}+M_{p}^{\mathcal{L}}} d|u| .
\end{aligned}
$$

Given $m_{1} \in \mathbb{R}$ and $m_{2}>0$, the function $[0, \infty) \ni x \mapsto H(x)=x^{m_{1}} \exp \left(-m_{2} \log ^{2}(x)\right)$ attains its maximum value $H\left(x_{0}\right)=\exp \left(\frac{m_{1}^{2}}{4 m_{2}}\right)$ at $x_{0}=\exp \left(\frac{m_{1}}{2 m_{2}}\right)$. This yields an upper bound for the 
integrand in (5.21); the expression in (5.21) is estimated from above by

$$
\begin{aligned}
& \tilde{\rho} \exp \left(\frac{\left(M_{p}^{\mathcal{L}}-3 / 2\right)^{2} \log (q)}{2\left(\kappa+k_{2}\right)}\right) \exp \left(\frac{1}{2 \log (q)}\left(\frac{k_{2}^{2}}{\kappa+k_{2}}-k_{2}+k_{1}\right) \log ^{2}|\epsilon|\right) \\
& \quad \times \exp \left(\frac{1}{2 \log (q)}\left(\frac{k_{2}^{2}}{\kappa+k_{2}}-k_{2}\right) \log ^{2}|t|\right)|t|^{\frac{k_{2}\left(M_{p}^{\mathcal{L}}-3 / 2\right)}{\kappa+k_{2}}} \\
& \quad \times \exp \left(\frac{1}{\log (q)}\left(\frac{k_{2}^{2}}{\kappa+k_{2}}-k_{2}\right) \log |\epsilon| \log |t|\right)|\epsilon|^{\frac{k_{2}\left(M_{p}^{\mathcal{L}}-3 / 2\right)}{\kappa+k_{2}}} .
\end{aligned}
$$

The second line in (5.22) is upper bounded for every $t$ because $\frac{k_{2}^{2}}{\kappa+k_{2}}<k_{2}$, and we also have an upper bound for $\exp \left(\frac{1}{\log (q)}\left(\frac{k_{2}^{2}}{\kappa+k_{2}}-k_{2}\right) \log |\epsilon| \log |t|\right)$ is 1. Regarding Definition 5.2 and taking into account that

$$
\frac{k_{2}^{2}}{\kappa+k_{2}}-k_{2}=-k_{1}
$$

expression (5.22) is upper bounded by

$$
K_{33}|\epsilon|^{\frac{k_{2}\left(M_{p}^{\mathcal{L}}-3 / 2\right)}{\kappa+k_{2}}}
$$

for some $K_{33}>0$. The conclusion is achieved. The result follows from (5.19), the estimates of $J_{1}$ to $J_{4}$, and (5.20).

\section{Existence of formal series solutions in the complex parameter and asymptotic expansion in two levels}

In the first part of this section, we recall two $q$-analogs of the Ramis-Sibuya theorem from $[7,13]$. This result provides a tool to guarantee the existence of a formal power series in the perturbation parameter, which formally solves the main problem and such that it asymptotically represents the analytic solution of that equation.

This asymptotic representation is held in the sense of $q$-asymptotic expansions of certain positive order.

Definition 6.1 Let $V$ be a bounded open sector with vertex at 0 in $\mathbb{C}$. Let $\left(\mathbb{F},\|\cdot\|_{\mathbb{F}}\right)$ be a complex Banach space. Let $q \in \mathbb{R}$ with $q>1$, and let $k$ be a positive integer. We say that a holomorphic function $f: V \rightarrow \mathbb{F}$ admits the formal power series $\hat{f}(\epsilon)=\sum_{n \geq 0} f_{n} \epsilon^{n} \in \mathbb{F} \llbracket \epsilon \rrbracket$ as its $q$-Gevrey asymptotic expansion of order $1 / k$ if for every open subsector $U$ with $(\bar{U} \backslash\{0\}) \subseteq V$, there exist $A, C>0$ such that

$$
\left\|f(\epsilon)-\sum_{n=0}^{N} f_{n} \epsilon^{n}\right\|_{\mathbb{F}} \leq C A^{N+1} q^{\frac{N(N+1)}{2 k}}|\epsilon|^{N+1}
$$

for all $\epsilon \in U$ and $N \geq 0$.

The set of functions that admit the null $q$-Gevrey asymptotic expansion of certain positive order are characterized as follows. The proof of this result, already stated in [13], provides the $q$-analog of Theorem XI-3-2 in [4]. 
Lemma 6.2 A holomorphic function $f: V \rightarrow \mathbb{F}$ admits the null formal power series $\hat{0} \in$ $\mathbb{F} \llbracket \epsilon \rrbracket$ as its $q$-Gevrey asymptotic expansion of order $1 / k$ if and only iffor every open subsector $U$ with $(\bar{U} \backslash\{0\}) \subseteq V$, there exist constants $K_{1} \in \mathbb{R}$ and $K_{2}>0$ such that

$$
\|f(\epsilon)\|_{\mathbb{F}} \leq K_{2} \exp \left(-\frac{k}{2 \log (q)} \log ^{2}|\epsilon|\right)|\epsilon|^{K_{1}}
$$

for all $\epsilon \in U$.

The next result is based on the one-level version of the $q$-analog of the Ramis-Sibuya theorem, stated in [13], and provides a two-level result in this framework. See [7] for a proof.

Theorem 6.3 Let $\left(\mathbb{F},\|\cdot\|_{\mathbb{F}}\right)$ be a Banach space, and let $\left(\mathcal{E}_{p}\right)_{0 \leq p \leq s-1}$ be a good covering in $\mathbb{C}^{\star}$. Let $0<k_{1}<k_{2}$, consider a holomorphic function $G_{p}: \mathcal{E}_{i} \rightarrow \mathbb{F}$ for $0 \leq p \leq \varsigma-1$, and put $\Delta_{p}(\epsilon)=G_{p+1}(\epsilon)-G_{p}(\epsilon)$ for $\epsilon \in Z_{p}:=\mathcal{E}_{p} \cap \mathcal{E}_{p+1}$. Moreover, we assume that:

(1) The functions $G_{p}(\epsilon)$ are bounded as $\epsilon$ tends to 0 on $\mathcal{E}_{p}$ for every $0 \leq p \leq \varsigma-1$.

(2) There exist nonempty sets $I_{1}, I_{2} \subseteq\{0,1, \ldots, \varsigma-1\}$ such that $I_{1} \cup I_{2}=\{0,1, \ldots, \varsigma-1\}$ and $I_{1} \cap I_{2}=\emptyset$. Also,

- for every $p \in I_{1}$, there exist constants $K_{1}>0$ and $M_{1} \in \mathbb{R}$ such that

$$
\left\|\Delta_{p}(\epsilon)\right\|_{\mathbb{F}} \leq K_{1}|\epsilon|^{M_{1}} \exp \left(-\frac{k_{1}}{2 \log (q)} \log ^{2}|\epsilon|\right), \quad \epsilon \in Z_{p}, \quad \text { and }
$$

- for every $p \in I_{2}$, there exist constants $K_{2}>0$ and $M_{2} \in \mathbb{R}$ such that

$$
\left\|\Delta_{p}(\epsilon)\right\|_{\mathbb{F}} \leq K_{2}|\epsilon|^{M_{2}} \exp \left(-\frac{k_{2}}{2 \log (q)} \log ^{2}|\epsilon|\right), \quad \epsilon \in Z_{p} .
$$

Then there exists a convergent power series a $(\epsilon) \in \mathbb{F}\{\epsilon\}$ defined on some neighborhood of the origin and $\hat{G}^{1}(\epsilon), \hat{G}^{2}(\epsilon) \in \mathbb{F} \llbracket \epsilon \rrbracket$ such that $G_{p}$ can be written in the form

$$
G_{p}(\epsilon)=a(\epsilon)+G_{p}^{1}(\epsilon)+G_{p}^{2}(\epsilon)
$$

where $G_{p}^{1}(\epsilon)$ is holomorphic on $\mathcal{E}_{p}$ and admits $\hat{G}^{1}(\epsilon)$ as its $q$-Gevrey asymptotic expansion of order $1 / k_{1}$ on $\mathcal{E}_{p}$ for every $p \in I_{1}$, and $G_{p}^{2}(\epsilon)$ is holomorphic on $\mathcal{E}_{p}$ and admits $\hat{G}^{2}(\epsilon)$ as its $q$-Gevrey asymptotic expansion of order $1 / k_{2}$ on $\mathcal{E}_{p}$ for every $p \in I_{2}$.

We conclude this section with the main result in the work in which we guarantee the existence of a formal solution of the main problem (5.1), written as a formal power series in the perturbation parameter, with coefficients in an appropriate Banach space, say $\hat{u}(t, z, \epsilon)$. Moreover, it represents, in some sense to be made precise, each solution $u^{\mathfrak{o}_{p}}(t, z, \epsilon)$ of problem (5.1).

From now on, $\mathbb{F}$ stands for the Banach space of bounded holomorphic functions defined on $\mathcal{T} \times H_{\beta^{\prime}}$ with the supremum norm, where $\beta^{\prime}<\beta$ as before.

Theorem 6.4 Under the hypotheses of Theorem 5.3, there exists a formal power series

$$
\hat{u}(t, z, \epsilon)=\sum_{m \geq 0} h_{m}(t, z) \frac{\epsilon^{m}}{m !} \in \mathbb{F} \llbracket \epsilon \rrbracket,
$$


a formal solution of the equation

$$
\begin{aligned}
& Q\left(\partial_{z}\right) \sigma_{q, t} \hat{u}(t, z, \epsilon) \\
& =(\epsilon t)^{d_{D_{1}}} \sigma_{q, t}^{\frac{d_{D_{1}}}{k_{1}}+1} R_{D_{1}}\left(\partial_{z}\right) \hat{u}(t, z, \epsilon)+(\epsilon t)^{d_{D_{2}}} \sigma_{q, t}^{\frac{d_{D_{2}}}{k_{2}}+1} R_{D_{2}}\left(\partial_{z}\right) \hat{u}(t, z, \epsilon) \\
& \quad+\sum_{\ell=1}^{D-1} \epsilon^{\Delta_{\ell}} t^{d_{\ell}} \sigma_{q, t}^{\delta_{\ell}}\left(c_{\ell}(t, z, \epsilon) R_{\ell}\left(\partial_{z}\right) \hat{u}(t, z, \epsilon)\right)+\sigma_{q, t} f(t, z, \epsilon) .
\end{aligned}
$$

Moreover, $\hat{u}(t, z, \epsilon)$ turns out to be the common $q$-Gevrey asymptotic expansion of order $1 / k_{1}$ on $\mathcal{E}_{p}$ of the function $u^{\mathrm{d}_{p}}$, seen as a holomorphic function from $\mathcal{E}_{p}$ into $\mathbb{F}$, for $0 \leq p \leq \varsigma-1$. In addition to that, $\hat{u}$ is of the form

$$
\hat{u}(t, z, \epsilon)=a(t, z, \epsilon)+\hat{u}_{1}(t, z, \epsilon)+\hat{u}_{2}(t, z, \epsilon)
$$

where $a(t, z, \epsilon) \in \mathbb{F}\{\epsilon\}$ and $\hat{u}_{1}(t, z, \epsilon), \hat{u}_{2}(t, z, \epsilon) \in \mathbb{F} \llbracket \epsilon \rrbracket$ are such that for every $0 \leq p \leq \varsigma-1$, the function $u^{\mathfrak{o}_{p}}$ can be written in the form

$$
u^{\mathfrak{d}_{p}}(t, z, \epsilon)=a(t, z, \epsilon)+u_{1}^{\mathfrak{d}_{p}}(t, z, \epsilon)+u_{2}^{\mathfrak{o}_{p}}(t, z, \epsilon)
$$

where $\epsilon \mapsto u_{1}^{\mathfrak{d}_{p}}(t, z, \epsilon)$ is an $\mathbb{F}$-valued function that admits $\hat{u}_{1}(t, z, \epsilon)$ as its $q$-Gevrey asymptotic expansion of order $1 / k_{1}$ on $\mathcal{E}_{p}$, and also $\epsilon \mapsto u_{2}^{\mathrm{o}_{p}}(t, z, \epsilon)$ is an $\mathbb{F}$-valued function that admits $\hat{u}_{2}(t, z, \epsilon)$ as its $q$-Gevrey asymptotic expansion of order $1 / k_{2}$ on $\mathcal{E}_{p}$.

Proof For every $0 \leq p \leq \varsigma-1$, we can consider the function $u^{\mathfrak{d}_{p}}(t, z, \epsilon)$ constructed in Theorem 5.3. We define $G_{p}(\epsilon):=(t, z) \mapsto u^{\mathfrak{d}_{p}}(t, z, \epsilon)$, which is a holomorphic bounded function from $\mathcal{E}_{p}$ into $\mathbb{F}$. In view of Propositions 5.4 and 5.6 , we can split the set $\{0,1, \ldots, \varsigma-1\}$ into two nonempty subsets of indices, $I_{1}$ and $I_{2}$ with $\{0,1, \ldots, \varsigma-1\}=I_{1} \cup I_{2}$ and such that $I_{1}$ (resp., $I_{2}$ ) consists of all the elements in $\{0,1, \ldots, \varsigma-1\}$ such that $U_{\mathfrak{d}_{p}} \cap U_{\mathfrak{o}_{p+1}}$ contains the sector $U_{\mathfrak{d}_{p}, \mathfrak{d}_{p+1}}$, as defined in Proposition 5.4 (resp., $U_{\mathfrak{d}_{p}} \cap U_{\mathfrak{o}_{p+1}}=\emptyset$ ). By (5.6) and (5.18) we can apply Theorem 6.3 and deduce the existence of formal power series $\hat{G}^{1}(\epsilon), \hat{G}^{2}(\epsilon) \in \mathbb{F} \llbracket \epsilon \rrbracket$, a convergent power series $a(\epsilon) \in \mathbb{F}\{\epsilon\}$, and holomorphic functions $G_{p}^{1}(\epsilon)$ and $G_{p}^{2}(\epsilon)$ defined on $\mathcal{E}_{p}$ with values in $\mathbb{F}$ such that

$$
G_{p}(\epsilon)=a(\epsilon)+G_{p}^{1}(\epsilon)+G_{p}^{2}(\epsilon)
$$

and for $j=1,2, G_{p}^{j}(\epsilon)$ admits $\hat{G}^{j}(\epsilon)$ as its $q$-Gevrey asymptotic expansion or order $1 / k_{j}$ on $\mathcal{E}_{p}$. We put

$$
\hat{u}(t, z, \epsilon)=\sum_{m \geq 0} h_{m}(t, z) \frac{\epsilon^{m}}{m !}:=a(\epsilon)+\hat{G}_{p}^{1}(\epsilon)+\hat{G}_{p}^{2}(\epsilon) .
$$

It only remains to prove that $\hat{u}(t, z, \epsilon)$ is a solution of (6.2). Indeed, since $u^{\mathfrak{d}_{p}}$ admits $\hat{u}(t, z, \epsilon)$ as its $q$-Gevrey asymptotic expansion of order $1 / k_{1}$ on $\mathcal{E}_{p}$, we have that

$$
\lim _{\epsilon \rightarrow 0, \epsilon \in \mathcal{E}_{p}} \sup _{t \in \mathcal{T}, z \in H_{\beta^{\prime}}}\left|\partial_{\epsilon}^{m} u^{\mathfrak{d}_{p}}(t, z, \epsilon)-h_{m}(t, z)\right|=0
$$


for all $0 \leq p \leq \varsigma-1$ and $m \geq 0$. Let $p \in\{0,1, \ldots, \varsigma-1\}$. By construction, the function $u^{\mathfrak{o}_{p}}(t, z, \epsilon)$ solves equation (6.2). We take the derivatives of order $m \geq 0$ with respect to $\epsilon$ of both sides of equation (5.1) and deduce that

$$
\begin{aligned}
& Q\left(\partial_{z}\right) \sigma_{q, t}\left(\partial_{\epsilon}^{m} u^{\mathrm{o}_{p}}\right)(t, z, \epsilon) \\
& =\sum_{m_{1}+m_{2}=m} \frac{m !}{m_{1} ! m_{2} !} \partial_{\epsilon}^{m_{1}}\left(\epsilon^{d_{D_{1}}}\right) t^{d_{D_{1}}} \sigma_{q, t}^{\frac{d_{D_{1}}}{k_{1}}+1} R_{D_{1}}\left(\partial_{z}\right)\left(\partial_{\epsilon}^{m_{2}} u^{\mathfrak{o}_{p}}\right) \\
& \quad+\sum_{m_{1}+m_{2}=m} \frac{m !}{m_{1} ! m_{2} !} \partial_{\epsilon}^{m_{1}}\left(\epsilon^{d_{D_{2}}}\right) t^{d_{D_{2}}} \sigma_{q, t}^{\frac{d_{D_{2}}}{k_{2}}+1} R_{D_{2}}\left(\partial_{z}\right)\left(\partial_{\epsilon}^{m_{2}} u^{\mathfrak{o}_{p}}\right) \\
& \quad+\sum_{\ell=1}^{D-1} \sum_{m_{1}+m_{2}+m_{3}=m} \frac{m !}{m_{1} ! m_{2} ! m_{3} !}\left(\partial_{\epsilon}^{m_{1}} \epsilon^{\Delta_{\ell}}\right) t^{d_{\ell}} \sigma_{q, t}^{\delta_{\ell}}\left(\partial_{\epsilon}^{m_{2}} c_{\ell}(t, z, \epsilon) R_{\ell}\left(\partial_{z}\right) \partial_{\epsilon}^{m_{3}} u^{\mathfrak{d}_{p}}(t, z, \epsilon)\right) \\
& \quad+\sigma_{q, t}\left(\partial_{\epsilon}^{m} f\right)(t, z, 0)
\end{aligned}
$$

for every $(t, z, \epsilon) \in \mathcal{T} \times H_{\beta^{\prime}} \times \mathcal{E}_{p}$. We let $\epsilon \rightarrow 0$ in (6.3) and obtain the recursion formula

$$
\begin{aligned}
& Q\left(\partial_{z}\right) \sigma_{q, t} h_{m}(t, z) \\
& =\frac{m !}{\left(m-d_{D_{1}}\right) !} t^{d_{D_{1}}} \sigma_{q, t}^{\frac{d_{D_{1}}}{k_{1}}+1} R_{D_{1}}\left(\partial_{z}\right)\left(h_{m-d_{D_{1}}}(t, z)\right) \\
& \quad+\frac{m !}{\left(m-d_{D_{2}}\right) !} t^{d_{D_{2}}} \sigma_{q, t}^{\frac{d_{D_{2}}}{k_{2}}+1} R_{D_{2}}\left(\partial_{z}\right)\left(h_{m-d_{D_{2}}}(t, z)\right) \\
& \quad+\sum_{\ell=1}^{D-1} \sum_{m_{2}+m_{3}=m-\Delta_{\ell}} \frac{m !}{m_{2} ! m_{3} !} t^{d_{\ell}} \sigma_{q, t}^{\delta_{\ell}}\left(\partial_{\epsilon}^{m_{2}} c_{\ell}(t, z, 0) R_{\ell}\left(\partial_{z}\right) h_{m_{3}}(t, z)\right) \\
& \quad+\sigma_{q, t}\left(\partial_{\epsilon}^{m} f\right)(t, z, 0)
\end{aligned}
$$

for all $m \geq \max \left\{d_{D_{1}}, d_{D_{2}}, \max _{1 \leq \ell \leq D-1} \Delta_{\ell}\right\}$ and $(t, z) \in \mathcal{T} \times H_{\beta^{\prime}}$.

Bearing in mind that both $c_{l}$ and $f$ are holomorphic with respect to $\epsilon$ in a neighborhood of the origin, in such neighborhood, we have

$$
c_{\ell}(t, z, \epsilon)=\sum_{m \geq 0} \frac{\left(\partial_{\epsilon}^{m} c_{m}\right)(t, z, 0)}{m !} \epsilon^{m}, \quad f(t, z, \epsilon)=\sum_{m \geq 0} \frac{\left(\partial_{\epsilon}^{m} f\right)(t, z, 0)}{m !} \epsilon^{m}
$$

for every $1 \leq \ell \leq D-1$.

By plugging (6.1) into (6.2) and bearing in mind (6.4) and (6.5) we conclude that the formal power series $\hat{u}(t, z, \epsilon)=\sum_{m \geq 0} h_{m}(t, z) \epsilon^{m} / m$ ! is a solution of equation (6.2).

\section{Acknowledgements}

We want to express our gratitude to the anonymous referee for the valuable comments and suggestions made, which helped us to improve the work.

\section{Funding}

This project has received funding from the European Research Council (ERC) under the European Union's Horizon 2020 research and innovation programme under the Grant Agreement No 648132. The second and third authors are partially supported by the research project MTM2016-77642-C2-1-P of Ministerio de Economía, Industria y Competitividad, Spain. 
Availability of data and materials

Data sharing is not applicable to this paper as no datasets were generated or analyzed during the current study.

\section{Competing interests}

The authors declare that they have no competing interests.

\section{Authors' contributions}

All authors contributed equally and significantly in writing this paper and typed, read, and approved the final manuscript.

\section{Author details}

'IRMA, Université de Strasbourg, Strasbourg, France. ${ }^{2}$ Departamento de Física y Matemáticas, University of Alcalá, Alcalá de Henares, Spain. ${ }^{3}$ Laboratoire Paul Painlevé, University of Lille 1, Villeneuve d'Ascq cedex, France.

\section{Publisher's Note}

Springer Nature remains neutral with regard to jurisdictional claims in published maps and institutional affiliations.

Received: 1 February 2019 Accepted: 25 July 2019 Published online: 07 August 2019

\section{References}

1. Costin, O., Tanveer, S.: Short time existence and Borel summability in the Navier-Stokes equation in $\mathbb{R}^{3}$. Commun. Partial Differ. Equ. 34(7-9), 785-817 (2009)

2. Di Vizio, L., Zhang, C.: On q-summation and confluence. Ann. Inst. Fourier (Grenoble) 59(1), 347-392 (2009)

3. Dreyfus, T.: Building meromorphic solutions of $q$-difference equations using a Borel-Laplace summation. Int. Math. Res. Not. 15, 6562-6587 (2015)

4. Hsieh, P.-F., Sibuya, Y.: Basic Theory of Ordinary Differential Equations. Universitext. Springer, New York (1999)

5. Lastra, A., Malek, S.: On q-Gevrey asymptotics for singularly perturbed q-difference-differential problems with an irregular singularity. Abstr. Appl. Anal. 2012, Article ID 860716 (2012)

6. Lastra, A., Malek, S.: On parametric Gevrey asymptotics for singularly perturbed partial differential equations with delays. Abstr. Appl. Anal. 2013, Article ID 723040 (2013)

7. Lastra, A., Malek, S.: On parametric multilevel q-Gevrey asymptotics for some linear q-difference-differential equations. Adv. Differ. Equ. 2015, 344 (2015)

8. Lastra, A., Malek, S.: On parametric multisummable formal solutions to some nonlinear initial value Cauchy problems. Adv. Differ. Equ. 2015, 200 (2015)

9. Lastra, A., Malek, S.: On multiscale Gevrey and $q$-Gevrey asymptotics for some linear $q$-difference differential initial value Cauchy problems. J. Differ. Equ. Appl. 23(8), 1397-1457 (2017)

10. Lastra, A., Malek, S., Sanz, J.: On q-asymptotics for linear q-difference-differential equations with Fuchsian and irregular singularities. J. Differ. Equ. 252(10), 5185-5216 (2012)

11. Malek, S.: On Gevrey asymptotics for some nonlinear integro-differential equations. J. Dyn. Control Syst. 16(3), 377-406 (2010)

12. Malek, S.: On singularly perturbed q-difference-differential equations with irregular singularity. J. Dyn. Control Syst. 17(2), 243-271 (2011)

13. Malek, S.: Parametric Gevrey asymptotics for a $q$-analog of some linear initial value problem. Funkc. Ekvacioj 60(1), 21-63 (2017)

14. Pravica, D., Randriampiry, N., Spurr, M.: q-Advanced models for tsunamis and rogue waves. Abstr. Appl. Anal. 2012, Article ID 414060 (2012)

15. Pravica, D., Randriampiry, N., Spurr, M.: On $q$-advanced spherical Bessel functions of the first kind and perturbations of the Haar wavelet. Appl. Comput. Harmon. Anal. 44(2), 350-413 (2018)

16. Pravica, D., Randriampiry, N., Spurr, M.: Solutions of a class of multiplicatively advanced differential equations. C. R. Math. Acad. Sci. Paris 356(7), 776-817 (2018)

17. Ramis, J.-P.: About the growth of entire functions solutions of linear algebraic $q$-difference equations. Ann. Fac. Sci. Toulouse Math. (6) 1(1), 53-94 (1992)

18. Tahara, H.: q-Analogues of Laplace and Borel transforms by means of q-exponentials. Ann. Inst. Fourier (Grenoble) 67(5), 1865-1903 (2017)

19. Tahara, H., Yamazawa, H.: Multisummability of formal solutions to the Cauchy problem for some linear partial differential equations. J. Differ. Equ. 255(10), 3592-3637 (2013)

20. Tahara, H., Yamazawa, H.: q-Analogue of summability of formal solutions of some linear $q$-difference-differential equations. Opusc. Math. 35(5), 713-738 (2015)

21. Yamazawa, H.: Holomorphic and singular solutions of q-difference-differential equations of Briot-Bouquet type. Funkc. Ekvacioj 59(2), 185-197 (2016)

22. Yamazawa, H.: Gevrey and q-Gevrey asymptotics for some linear q-difference-differential equations, communication in "Formal and analytic solutions of functional equations on the complex domain", Kyoto (2018) 